\title{
Las aves del departamento de Loreto, Perú
}

\author{
The birds from Department of Loreto, Peru
}

\section{Letty Salinas* 1, 2 \\ https://orcid.org/0000-0003-1002-595X \\ Isalinass@unmsm.edu.pe

\author{
https://orcid.org/0000-0003-1046-3343 \\ alejandra.arana@unmsm.edu.pe \\ César Arana 1,3 \\ https://orcid.org/0000-0002-7566-5205 \\ caranab@unmsm.edu.pe
} \\ Alejandra Arana ${ }^{1,2}$ \\ *Corresponding author}

1 Universidad Nacional Mayor de San Marcos, Facultad de Ciencias Biológicas, Lima, Perú.

2 Universidad Nacional Mayor de San Marcos, Museo de Historia Natural, Departamento de Ornitología, Lima, Perú.

3 Universidad Nacional Mayor de San Marcos, Museo de Historia Natural, Departamento de Ecología, Lima, Perú.

\section{Citación}

Salinas L, Arana A, Arana C. 2021. Las aves del departamento de Loreto. Revista peruana de biología 28(especial): e21915 001- 040 (Diciembre 2021). doi: http://dx.doi. org/10.15381/rpb.v28iespecial.21915

\section{Presentado: $\quad 09 / 02 / 2021$ \\ Aceptado: $\quad 10 / 08 / 2021$ \\ Publicado online: 30/12/2021}

Editor:

Leonardo Romero

\section{Resumen}

Presentamos una lista actualizada de las especies de aves del departamento de Loreto. La lista está basada en la revisión de ejemplares de colecciones científicas, registros históricos, publicaciones y observaciones de campo. Reportamos 1040 especies de aves para Loreto, 72 de ellas en alguna categoría de amenaza, 72 migratorias y 13 endémicas de Perú. Percnostola arenarum, Pithys castaneus y Polioptila clementsi son endémicas de Loreto. Las familias con mayor número de especies son Tyrannidae, Thamnophilidae y Thraupidae. La lista presentada tiene alta confiabilidad debido al respaldo de cerca del $81 \%$ de las especies en especímenes de colecciones científicas y apenas el $1.5 \%$ solo por reportes de avistamientos realizados por ornitólogos. Las especies de aves de Loreto representan alrededor del 55\% del total reportado para el Perú y en Passeriformes el 52\% de los estimado para la Amazonia. La distribución de los registros muestra que es necesario explorar áreas cercanas a las fronteras con Colombia y Brasil, así como las cordilleras que comparten con Amazonas y San Martín. En base a la distribución de la avifauna amenazada y endémica se propone priorizar el estudio y protección de áreas montanas y de bosques de arena blanca en particular.

\section{Abstract}

We present an updated list of bird species from Department of Loreto. The list is based on the review of specimens from scientific collections, historical records, publications, and field observations. We report 1040 species of birds in Loreto, 72 in some threat category, 72 migratory and 13 endemics to Peru. Percnostola arenarum, Pithys castaneus and Polioptila clementsi are endemics to Loreto. The families with the highest numbers of species are Tyrannidae, Thamnophilidae and Thraupidae. The list presented is highly reliable due to the support of about $81 \%$ of the species on specimens from scientific collections and only $1.5 \%$ from sighting reports by ornithologists. Bird species from Loreto represent about $55 \%$ of the total reported for Peru and in Passeriformes $52 \%$ of those estimated for the Amazonia. The distribution of reports shows that it is necessary to explore areas close to the region's borders with Colombia and Brazil, as well as the mountain ranges shared with Amazonas and San Martin. Based on the distribution of threatened and endemic avifauna, we propose to prioritize the study and protection of montane areas and white-sand forests.

Palabras clave:

Amazonia; colecciones científicas; Neotrópico; Sudamérica; Perú.

Keywords:

Amazonia; Neotropics; scientific collections; South America; Peru. 


\section{Introducción}

Las aves de Loreto han sido objeto de estudio desde hace casi dos siglos (Sharpe 1874), encontrándose registros tempranos en colecciones científicas de Europa, los Estados Unidos de Norteamérica y también del Perú. Si bien en sus inicios los estudios estuvieron orientados al descubrimiento de nuevas especies y subespecies, con el tiempo los especímenes colectados en Loreto fueron los elementos centrales de investigaciones importantes como: el efecto de los ríos sobre la especiación (Remsen \& Parker III 1983, Armacost \& Capparella 2012), la alta especialización al ambiente (Rosenberg 1990), los gremios de alimentación (Remsen et al. 1993, Martínez \& Zenil 2012), las bandadas mixtas e interacción de especies (Martínez \& Robinson 2016), la muda y la reproducción (Torres-Vásquez et al. 2018) y la selección del hábitat (Ungvari Martin et al. 2016), entre los más notables. Por otro lado, las investigaciones moleculares subyacentes en las investigaciones taxonómicas y filogenéticas, genética de poblaciones (Burney \& Brumfield 2009, Harvey et al. 2017), evolución (Harvey et al. 2020), así como las expediciones a localidades históricas de colecta de mediados del siglo XIX (Moncrieff et al. 2019), han aportado a una mejor comprensión de la composición de especies en Loreto y sus historias evolutivas.

En los últimos años en Loreto se han realizado estudios a largo plazo en ámbitos geográficos extensos; por ejemplo, el conducido por José Álvarez en los ríos Nanay, Corrientes y Tigre (Díaz-Alván et al. 2017). También debe recalcarse el rol que ha tenido del Instituto de Investigación de la Amazonia Peruana (IIAP) en las últimas décadas con sus investigaciones institucionales y cooperativas en ámbitos con vacíos de información como en el Putumayo y Napo (Angulo-Pérez et al. 2018, Pérez-Peña et al. 2019). Además, existe una participación activa de investigadores nacionales en el reporte y documentación de ampliación de rango geográfico de aves ya registradas en los países vecinos (Acosta Diaz et al. 2019, Ruiz-Ramos et al. 2020, Vásquez-Arévalo et al. 2020).

El Field Museum of Natural History de Chicago ha tenido un importante papel en la investigación de la biodiversidad en Loreto a través de sus Inventarios biológicos rápidos o Rapid Assessment Program (Pitman et al. 2021). Desde el 2000, estos estudios han generado información (Schulenberg et al. 2001, Lane et al. 2003, Schulenberg et al. 2006, Stotz \& Pequeño 2006, Stotz \& Díaz-Alván 2007, Stotz \& Díaz-Alván 2010, Stotz \& Ruelas Inzunza 2013, Ruelas Inzunza et al. 2014, O'Shea et al. 2015, Stotz et al. 2016) que ha coadyuvado a la creación de 11 nuevas áreas protegidas. Estas nuevas áreas representan el 89\% de la superficie bajo protección en Loreto, y ha permitido que un $24 \%$ del área total del departamento de Loreto se encuentre bajo algún régimen de protección (Pitman et al. 2021).

Estas nuevas áreas de conservación y la promoción de un modelo de "conservación productiva" trabajando con las cámaras de comercio de los pueblos indígenas y la coordinadora de comunidades nativas y campesinas, han repotenciado las oportunidades del aviturismo (Al- varez Alonso 2010, Alvarez Alonso et al. 2012). Sin embargo, para que estas oportunidades se concreticen es necesario tener una lista de las aves del departamento con una alta confiabilidad y que permita llevar al mercado especializado del aviturismo una oferta de especies posibles de observar que cuente con respaldo científico.

Las propuestas de listas de aves de Loreto existentes varían en número de especies según los sistemas de clasificación utilizados. Para IOC, HBW, Clements y SACC se dan valores de 1086, 1088, 1086 y 1086 especies, respectivamente (Avibase 2020). Sin embargo, estas listas se producen a partir de bases de datos a nivel mundial y no detallan información precisa sobre cuáles especies han sido registradas en el área o han sido incluidas por proyecciones de sus probables distribuciones geográficas. Otras listas para Loreto indican entre registradas y esperadas un total de 1066 especies (Pitman et al. 2013) y 809 especies para las aves de Loreto bajo (Wiley et al. 2014), basándose la primera principalmente en la proyección de los mapas de distribución del libro Birds of Peru (Schulenberg et al. 2007) y la segunda en registros de avistamientos. El descubrimiento de nuevas especies, nuevos registros o subespecies es continuo (Whitney \& Alvarez Alonso 2005, Whitney \& Cohn-Haft 2013, O'Neill et al. 2000), con especies endémicas como el Machaeropterus eckelberryi (Lane et al. 2017), migrantes escasamente conocidos como el Oporornis agilis (Ungvari Martin 2017) o subespecies como Heliangelus regalis johnsoni (Graves et al. 2011). También se han realizado algunos exhaustivos registros de la avifauna en investigaciones de sistemática, distribución e historia de vida como en el redescubrimiento de Pithys castaneus en el río Morona (Lane et al. 2006) o ampliaciones de rango de distribución de aves comunes (Ugarte \& Lavalle Valdivia 2018, Pulido et al. 2021).

Con la finalidad de proporcionar información de alta confiabilidad, necesaria tanto para investigaciones básicas como aplicadas, el presente trabajo se planteó el objetivo de elaborar una lista actualizada de las especies de aves del departamento de Loreto, en base a una revisión crítica de diversas fuentes de información, en especial datos de colecciones científicas.

\section{Material y métodos}

Área de estudio. - Loreto es el departamento más grande del Perú. Se ubica en el extremo norte del país entre las coordenadas $0^{\circ} 02^{\prime} 37^{\prime \prime S}$ y 75¹0'29"W (río Putumayo cerca de $12 \mathrm{~km}$ NE de la desembocadura del río Güeppi) hacia el Norte, $13^{\circ} 20^{\prime} 04^{\prime \prime} \mathrm{S}$ y 69³8'38”W (cumbre de las nacientes de los ríos Pisqui, Santa Ana y Aspusana) hacia el Sur, 12³0'11"S y 68³9'27"W (río Amazonas, al sur del centro poblado Ramón Castilla) hacia

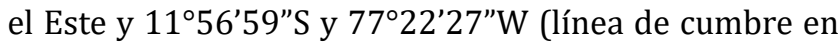
la naciente de los ríos Numpatque y tributarios del río Nieva) hacia el Oeste (INEI 2017, Fig. 1).

Loreto ocupa un área de $368851 \mathrm{~km}^{2}$, que corresponde al $28.7 \%$ de la superficie total del Perú, esta inserto en la parte nororiental del país, con altitudes menores a $250 \mathrm{~m}$ en la gran mayoría de su territorio, pero en los límites con 
los departamentos de Amazonas (cordillera Kampankis) y San Martín (cordillera Escalera) se pueden alcanzar altitudes de 1400 y 2000 m respectivamente (Fig. 1).

Loreto se encuentra entre dos grandes unidades morfoestructurales, la cordillera de los Andes y el Escudo Brasileño, ocupando en su gran mayoría un área de suave fisiografía colinosa y zonas aluviales (llanura amazónica) surcada por numerosos ríos y con un área hacia el oeste con montañas que alcanzan alrededor de los $2000 \mathrm{~m}$ de altitud (Fig. 1). El clima de Loreto es cálido, con alta humedad relativa y altas precipitaciones con valores de temperatura media anual de alrededor de $26.5^{\circ} \mathrm{C}$ y precipitaciones anuales de alrededor de 2574 mm (Fig. 1).

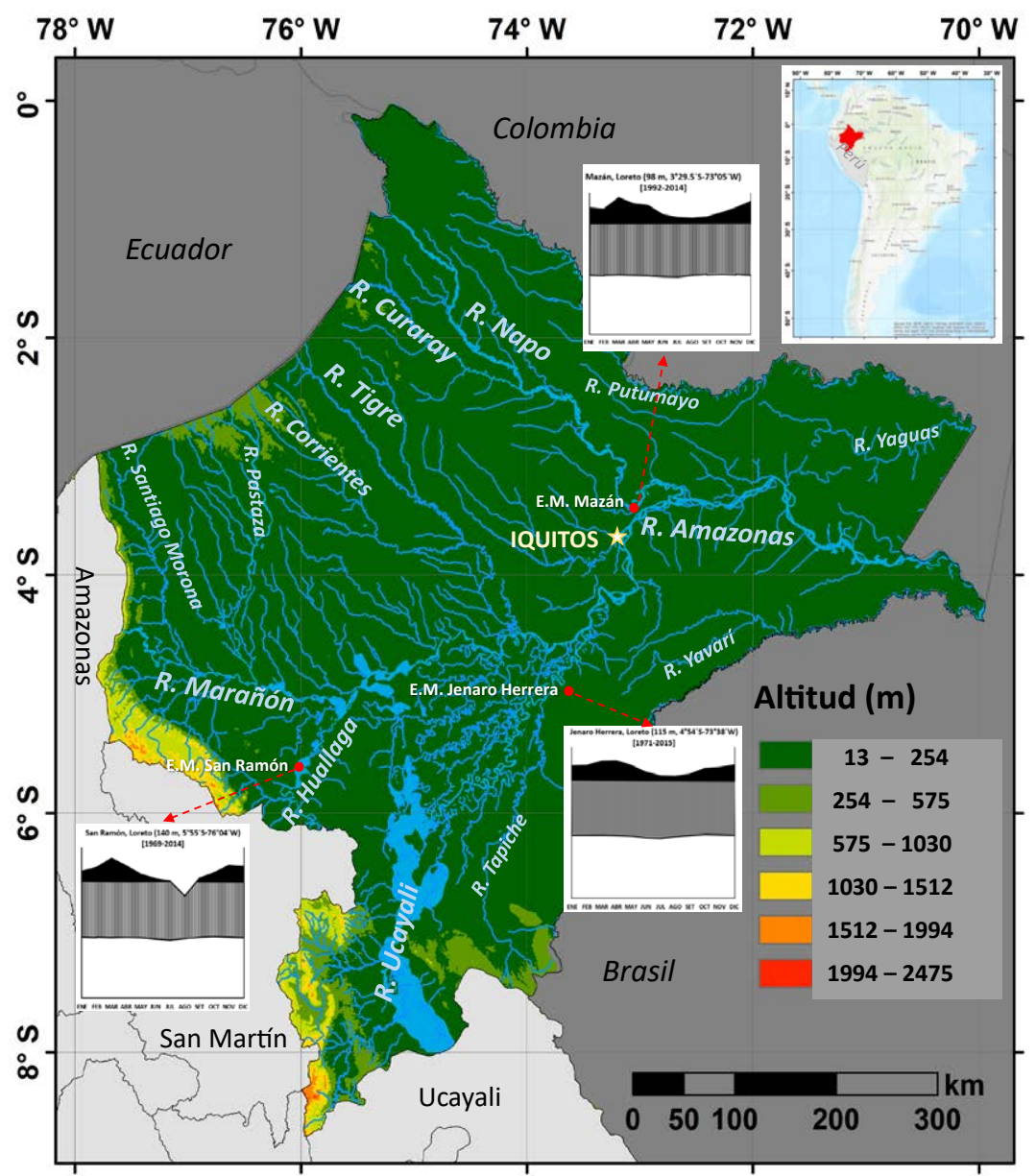

Figura 1. Departamento de Loreto, variación de la altitud, principales ríos (R.), ubicación de las tres estaciones meteorológicas cuyos diagramas ombrotérmicos son mostrados (E.M.) y ubicación de la capital Iquitos.

Loreto pertenece a la región denominada "parte norteña de la Amazonia occidental" (Ter Steege et al. 2013). Es una región con una alta diversidad de plantas y heterogeneidad de bosques, incluyendo bosques de colina baja (que cubre la mayor parte de la superficie de la región), bosques inundables de palmeras, bosques de terraza baja y de terraza alta, herbazales hidrofíticos, bosques de montaña, bosques de montaña basimontanos, bosques de colina alta, bosques de llanura meándrica, vegetación esclerófila de arena blanca, vegetación de isla, bosques de terraza inundables por aguas negras y áreas deforestadas (MINAM 2015). Loreto también incluye cinco provincias biogeográficas, las provincias de Napo, Imerí, Ucayali, Madeira y Rondônia, las dos primeras pertenecientes al dominio Bra- siliano Boreal y las tres últimas al dominio Brasiliano del Sur (Morrone 2014).

Elaboración y validación de la lista de especies. Para la elaboración de la lista de especies se realizó una base de datos primaria a partir de la revisión de los especímenes correspondientes a Loreto de la Colección Ornitológica del Museo de Historia Natural de la Universidad Nacional Mayor de San Marcos. Luego se añadieron especies con evidencia de su presencia en Loreto (espécimen, foto o audio) en otras colecciones científicas que cuentan con información digitalizada en las plataformas del Smithsonian National Museum of Natural History (NMNH 2020), del American Museum of Natural History (AMNH 2020), la Global Biodiversity 
Information Facility (GBIF 2020), Integrated Digitized Biocollections (iDigBio 2020) y VertNet (VertNet 2020). Se incluyeron también datos de los inventarios de colecciones científicas publicados (Sharpe 1874, Brabourne \& Chubb 1917, Hastert 1918, Zimmer 1924, Zimmer 1937, van den Hoek Ostende et al. 1997, LeCroy \& Sloss 2000, Roselaar 2003, Lecroy 2005, Mlíkovský 2009, Lecroy 2012). Se obtuvo información procedente de 30 colecciones científicas, de las cuales 21 están ubicadas en Norteamérica, seis en Europa y tres en Sudamérica. Las colecciones científicas revisadas y sus respectivos acrónimos están detalladas en el Anexo 1.

A esta información se sumaron los registros publicados. Para la búsqueda bibliográfica se utilizaron los buscadores Google, Google Scholar y Scopus; utilizando diferentes combinaciones de las palabras clave: Loreto, birds, Amazonia, Aves, río Tigre, río Nanay, río Napo, río Amazonas, río Ucayali, río Marañón, río Huallaga, río Pastaza, río Corrientes, río Putumayo, río Yavarí, avifauna, Allpahuayo Mishana, Matsés, Pacaya-Samiria, Pucacuro, Santiago-Comaina, Sierra del Divisor, Huimeki, Airo Pai, Güeppí-Sekime, Yaguas, Iquitos, Yurimaguas, Nauta y Requena. Entre las publicaciones obtenidas en esta búsqueda, destacan las recopiladas en Biodiversity Heritage Library (BHL 2020) que permitieron registrar numerosos especímenes de valor histórico (anteriores al siglo XX) (Sclater 1857b, Sclater \& Salvin 1867, Sharpe 1874, Bartlett 1882, Taczanowski 1884a, 1884b, 1886).

Se revisaron libros sobre la avifauna de áreas dentro de Loreto (Schulenberg et al. 2007, Pitman et al. 2013, Naranjo et al. 2016, Angulo-Pérez et al. 2018, PérezPeña et al. 2019). También los resultados de los Rapid Biological and Social Inventories de The Field Museum realizados en Loreto (Schulenberg et al. 2001, Lane et al. 2003, Schulenberg et al. 2006, Stotz \& Pequeño 2006, Stotz \& Díaz-Alván 2007, Stotz \& Díaz-Alván 2010, Stotz \& Ruelas Inzunza 2013, Ruelas Inzunza et al. 2014, O'Shea et al. 2015, Stotz et al. 2016). Asimismo, se revisaron listas de libre acceso sobre aves del departamento (Wiley et al. 2014, Foss et al. 2021). Además, se revisaron los artículos sobre la avifauna, de las áreas protegidas y de nuevos registros de especies de Loreto (Salazar et al. 2003, Armacost Junior 2006, Shahuano Tello et al. 2008, Lüthi 2011, O’Neill et al. 2011, Álvarez Alonso et al. 2012, Bicerra Canayo 2013, García Bravo \& Barrio 2014, Díaz-Alván et al. 2017, Schmitt et al. 2017, Socolar et al. 2018, Ugarte \& Lavalle Valdivia 2018, Acosta Diaz et al. 2019, Moncrieff et al. 2019, Fong Rengifo et al. 2020, Garcia Solsol et al. 2020, Ruiz-Ramos et al. 2020, Vásquez-Arévalo et al. 2020).

Para incluir cada especie en la lista final, se verificó que la localidad de los especímenes de museos fuera correcta y no un error en las coordenadas; y se realizó una corroboración de observaciones actuales de estas especies en Loreto, utilizando información no publicada de los autores (2008 - 2016) y de ornitólogos reconocidos disponibles en la plataforma eBird (eBird 2020) y NatureServe (NatureServe 2020).

La lista presenta una columna de Validación en la cual se señala si la inclusión se basa en la presencia de un espécimen en una colección científica o "voucher" (V), en la mención de la especie en una publicación (P) o en un registro ya sea por expediciones de los autores o disponible en plataformas libres (R).

Se agrega un asterisco a la $\mathrm{V}\left(\mathrm{V}^{*}\right)$ cuando la especie cuenta con uno o más especímenes de valor histórico en alguna colección ornitológica. Se define en el presente trabajo a un espécimen de valor histórico como aquel correspondiente a expediciones realizadas antes del siglo $X X$. Por otro lado, se agregó una t a la V (Vt) en la especie que presenta un espécimen que ha sido colectado en Loreto, pero cuya distribución no corresponde al departamento y su presencia podría deberse a una introducción al área por el tráfico ilegal de vida silvestre.

Finalmente, son denominadas Esperadas (E) aquellas especies con registros con alto grado de certeza en localidades muy cercanas a Loreto y en ambiente similares a los presentes dentro del departamento.

La nomenclatura y clasificación taxonómica sigue la lista publicada del Comité de Clasificación de Sudamérica (Remsen et al. 2020) en su versión más reciente a la fecha de elaboración de la publicación (23 de diciembre de 2020).

Categorías de amenaza, especies migratorias y endemismo. - Se determinaron las categorías de amenaza actualizadas de las especies según la Unión Internacional para la Conservación de la Naturaleza (IUCN 2020) y la legislación del Perú (Decreto Supremo $N^{\circ}$ 004-2014-MINAGRI). Asimismo, se revisó qué especies están incluidas en los apéndices de la Convención sobre el Comercio Internacional de Especies Amenazadas de Fauna y Flora Silvestres (CITES 2020) y la Convención sobre la Conservación de las Especies Migratorias de Animales Silvestres (CMS 2020). Finalmente, el estatus de las especies como endémicas para Perú se obtuvo de la Lista de las Aves del Perú (Plenge 2021).

\section{Resultados}

Composición taxonómica. - Se registraron 1040 especies de aves para Loreto (Tabla 1). Estas especies están incluidas en 27 órdenes y 74 familias, con el orden Passeriformes incluyendo más de la mitad de las especies (608 spp., 58.5\%), seguido de lejos por los Apodiformes (75 spp., 7.2\%). Las familias con mayor número de especies fueron los Tyrannidae (13.3\%), Thamnophilidade (8.9\%), Thraupidae (8.6\%), Furnariidae (7.0\%) y Trochilidae (5.9\%), seguidas de otras 14 familias con más de 14 especies y 55 con 14 o menos especies, 20 de las cuales solo estuvieron representadas por una especie (Fig. 2). De este total, 72 especies son migratorias, 19 migrantes australes y 53 boreales.

El número de especies de aves reportado en el presente artículo es menor que el señalado por Pitman (Pitman et al. 2013) y por las listas de Avibase (Avibase 2020). Esto es consecuencia del uso de criterios más estrictos para la inclusión de las especies en la presente lista. La inclusión se basa principalmente en la existencia de vou- 
chers (838 spp., 80.6\%), registros en publicaciones (182 spp., $17.5 \%$ ) y con pocas excepciones se incluyen avistamientos de ornitólogos de reconocida experiencia (16 spp., 1.5\%). Cabe resaltar que en el trabajo de Pitman et al. (2013), un número alto de especies fueron consideradas "esperadas" (152) principalmente por proyección de los mapas del libro Birds of Peru de Schulenberg et al. (2007), sin embargo, muchas de estas especies son de distribución montana o de la Amazonia sur, y no tienen registros en las cercanías a Loreto, por lo que no han sido consideradas en el presente trabajo. En nuestra lista hemos logrado obtener evidencia para 1001 especies e incluimos solo 39 en la categoría de esperadas. Incluso la consideración para incluirlas en esta categoría requirió evidencia confiable de su presencia en localidades cercanas a Loreto y de hábitats similares.

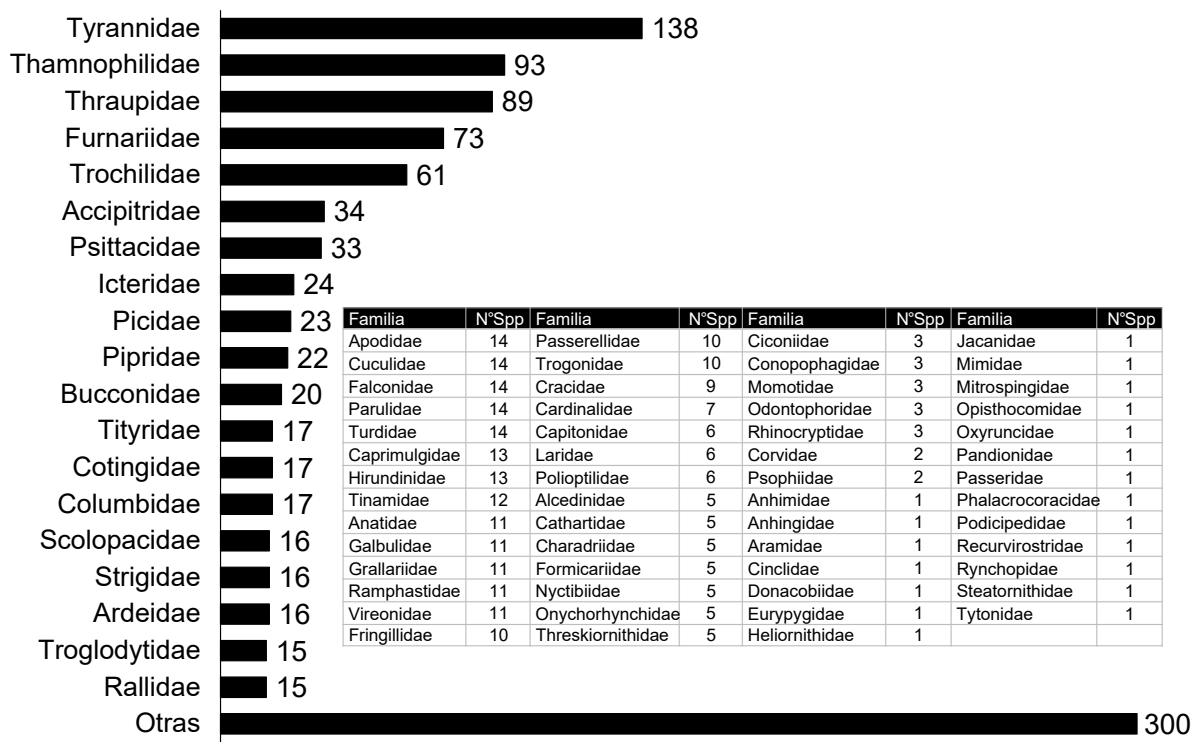

Figura 2. Número de especies por familia de aves registradas para Loreto, Perú. La tabla muestra las familias con menos de 15 especies (la categoría "Otras" del gráfico).

La avifauna de Loreto representa aproximadamente el 55.32\% de las especies de aves registradas para Perú (Plenge 2020), a pesar de solo incluir el 28.7\% de su superficie. El estado de Brasil más cercano y que tiene más de cuatro veces su superficie (Amazonas, casi un millón 600 mil km²) presenta alrededor de 1213 especies de aves (Avibase 2020), es decir unas 173 especies más que lo registrado para Loreto, mientras que el estado de Matto Grosso do Sul (de cerca del tamaño de Loreto, más de $358 \mathrm{mil} \mathrm{km}^{2}$ ) presenta 881 especies (Avibase 2020), es decir 159 especies menos. La comparación con países de otras latitudes con superficies similares a Loreto, como Alemania (más de $357 \mathrm{mil} \mathrm{km}^{2}$ ) con alrededor de 544 especies o Japón (casi 378 mil km²) con 719 especies, muestra la alta riqueza de especies de aves concentrada en este departamento del nororiente peruano. Considerando los Passeriformes en Loreto se registraron 608 especies, el $52.2 \%$ del total (1164 spp.) estimado para la Amazonia (Fluck et al. 2020).

Un grupo de especies consideradas "esperadas" tienen registros en zonas limítrofes con Loreto en Ecuador, Brasil y Colombia, con distribución altitudinal y características de hábitat similares a las de esta región. Aglaeactis cupripennis fue colectada en 1939 en el río Pichincha (Ecuador) y el espécimen de piel se encuentra en el $\mathrm{Na}$ tural History Museum of Los Angeles County. Pardirallus nigricans presenta registros de audio y avistamientos en el río Pastaza (Ecuador) con una distribución altitudinal (de 400 a 1050 m) coincidente con la de Loreto. Empidonax traillii, también es considerada esperada en la Lista de Aves de Loreto Bajo (Wiley et al. 2014), presentando observaciones en Leticia, Lagartococha y laguna de Bermeo (Colombia) y en el río Napo (Ecuador) en zonas limítrofes a Loreto. Myrmotherula sunensis tiene registros de audio en el río Pastaza y en Lagartococha (Ecuador), así como un avistamiento en el Parque Nacional La Paya en Colombia. Myiozetetes cayanensis cuenta con observaciones en localidades que limitan con Loreto en Brasil en el río Yavarí (Brasil) y en Leticia (Colombia).

Asimismo, Patagioenas fasciata, presente en bosques montanos de la vertiente oriental de los Andes, tiene un registro en Lagartococha (Colombia). Pygochelidon melanoleuca tiene registros cerca de Loreto dentro de Brasil y en el Parque Nacional Natural Amacayacu en Colombia, y es considerada como probable en la lista de Aves de Loreto Bajo (Wiley et al. 2014). Cantorchilus griseus es una especie vagrante, con registros en el río Yavarí (Brasil). Chaetura meridionalis, reportada cerca de la frontera con Brasil, posiblemente puede ocurrir en Perú según la Lista de las Aves del Perú (Plenge 2021). Chaetura spinicaudus tiene registros en la frontera con Brasil y es considerada como un registro nuevo para Perú en la Lista de Aves de Loreto bajo (Wiley et al. 2014). Chrysuronia versicolor tiene varios registros en localidades de Colombia y Brasil fronterizas con Loreto y es considerada probable en la Lista de Aves de Loreto bajo (Wiley et al. 2014). Myr- 
motherula iheringi se encuentra en el río Yavarí (Brasil) en dos localidades inmediatas a Loreto. Columbina passerina tiene registros en Leticia y en el río Yavarí (Brasil) en la frontera con Loreto. Vireo altiloquus es una especie vagrante, registrada como probable en la lista de Aves de Loreto bajo (Wiley et al. 2014) y que tiene colecta en la Isla Ronda (Colombia) en la frontera con Loreto, el ejemplar se encuentra en el Museum of Comparative Zoology, Harvard University. Lochmias nematura ha sido colectado en el río Pastaza (Ecuador) en la frontera con Loreto, con el espécimen depositado en la colección del Museum of Comparative Zoology, Harvard University; así como múltiples observaciones en el departamento de San Martín.

Otras especies consideradas en el presente estudio como "esperadas" han sido registradas en los departamentos de Amazonas o San Martín en localidades cercanas a la frontera con Loreto. Adicionalmente, presentan una distribución altitudinal y características del hábitat que coinciden con las presentes en Loreto. Amblycercus holosericeus es una especie montana con registros cerca de Moyobamba y Tarapoto. Campephilus haematogaster tiene registros en Amazonas, su rango de distribución altitudinal coincide con áreas de Loreto y tiene un voucher de Louisiana State University Museum of Natural Science cuyos detalles de localidad no han podido ser confirmados. Casiornis rufus es una especie migrante, con registros en San Martín. Chlorospingus canigularis tiene una distribución fragmentada en Perú, y está registrada en Amazonas, cerca de la frontera con Loreto. Colibri coruscans tiene registro en San Martín cerca de Balsapuerto (Loreto) y con distribución altitudinal desde los $400 \mathrm{~m}$. Conopias cinchoneti tiene registros en San Martín cerca de la frontera de Loreto y con distribución altitudinal similar. Coryphospingus cucullatus tiene registros cerca de Loreto en Amazonas y en Ecuador. Eriocnemis aline tiene registro en San Martín, en el Bosque de Protección Altomayo, así como un espécimen del Museum of Comparative Zoology, Harvard University cuya localidad está por confirmar.

Eubucco tucinkae tiene registros en Ucayali cerca de la frontera con Loreto y según los mapas de aves del libro Birds of Peru su área de distribución probable incluye Loreto (Schulenberg et al. 2007). Pharomachrus auriceps tiene registros en San Martín cerca de la frontera con Loreto. Lophornis stictolophus ha sido reportado para Amazonas en ambientes similares a Loreto. Setophaga fusca es una especie migratoria, registrada cerca de Loreto tanto en el Área de conservación regional Cordillera Escalera en San Martín, como en localidades ecuatorianas vecinas, incluyendo un ejemplar de Andoas, río Pastaza en el Moore Laboratory of Zoology. Pyrrhura rupicola tiene un registro en el Parque Nacional Sierra del Divisor en Ucayali cerca al límite con Loreto y es considerada como probable en la Lista de Aves de Loreto Bajo (Wiley et al. 2014). Myiophobus cryptoxanthus y Urochroa leucura han sido observadas cerca de la frontera de San Martín con Loreto, en la Reserva Ecológica Arena Blanca (Aguas Verdes). Chlorophonia cyanocephala tiene múltiples observaciones en localidades cercanas a Loreto en Amazonas y San Martín, en particular una grabación de Daniel Lane en Selva Andina cerca de $2 \mathrm{~km}$ del límite con Loreto.
Grallaria carrikeri, Grallaria squamigera y Thamnophilus ruficapillus son consideradas "esperadas" debido a que no tienen registros actuales, pero tienen registros históricos en Loreto detallados en el libro Ornithologie $d u$ Pérou (Taczanowski 1884a, 1884b, 1886). Grallaria squamigera también tiene un espécimen con localidad por confirmar en el Museum of Comparative Zoology, Harvard University. Otras especies incluidas tienen registros en colecciones científicas cuya localidad es incierta, como $\mathrm{He}$ mitriccus flammulatus, Muscisaxicola albilora, Ochthoeca rufipectoralis y Phyllomyias nigrocapillus con especímenes en el Museum of Comparative Zoology, Harvard University; e Iridophanes pulcherrimus que tiene un espécimen en el Louisiana State University Museum of Natural Science.

A pesar de la larga historia de colectas de aves en Loreto, solo algunas localidades tienen alto número de especímenes recolectados (al norte de la ciudad de Iquitos en el río Amazonas y en el Parque Nacional Cordillera Azul en el límite con San Martín), mientras que en la mayoría de las localidades el número de especímenes ha sido bajo (Fig. 3). Esta distribución de los datos de los especímenes de aves en Loreto en colecciones científicas muestra la necesidad de priorizar información documentada de la composición de especies en algunas zonas en particular (Fig. 3). Son áreas de prioridad las zonas limítrofes con Ecuador (norte de los distritos de Napo, Tigre, Monora), Colombia (distritos de Putumayo y Ramón Castilla) y Brasil (distritos de Yaquerana y Alto Tapiche), además de las áreas con mayores altitudes en los límites con las regiones de Amazonas (oeste de los distritos de Morona y Manseriche) y San Martín (al sur de los distritos de Barranca, Balsapuerto y Contamana).

\section{Especies en categorías de amenaza y endémicas}

La avifauna de Loreto incluye 29 especies que se encuentran en alguna categoría de amenaza según la IUCN (2020), 25 en categoría Vulnerable y cuatro en categoría En Peligro (Tabla 1). Las cuatro especies En Peligro son el paujil Crax globulosa, el picaflor Heliangelus regalis, el loro Pionites leucogaster y el hormiguerito Herpsilochmus parkeri. Adicionalmente, 43 especies de aves de Loreto se encuentran en la categoría de Casi Amenazada de la IUCN (2020), lo que indica una posible futura amenaza sobre sus poblaciones. En el ámbito nacional 23 especies están incluidas en categorías de amenaza (DS 004-2014-MINAGRI), 20 en Vulnerable, una En Peligro y dos En Peligro Crítico (Tabla 1). Las dos especies en Peligro Crítico son el paujil Crax globulosa y la reinita Polioptila clementsi, mientras que la especie en categoría En Peligro es el hormiguerito Herpsilochmus parkeri. Adicionalmente, 27 especies están consideradas como Casi Amenazadas. De este importante grupo de especies que requieren urgente investigación y acciones de conservación, destaca en especial Polioptila clementsi (Iquitos Gnatcatcher o Reinita de Iquitos), una especie endémica de los bosques de arena blanca de la reserva nacional Allpahuayo-Mishana que presenta una alta especialización a bosques con árboles altos donde se alimenta en las zonas altas del dosel y con una baja abundancia (Whitney \& Alvarez Alonso 2005). 


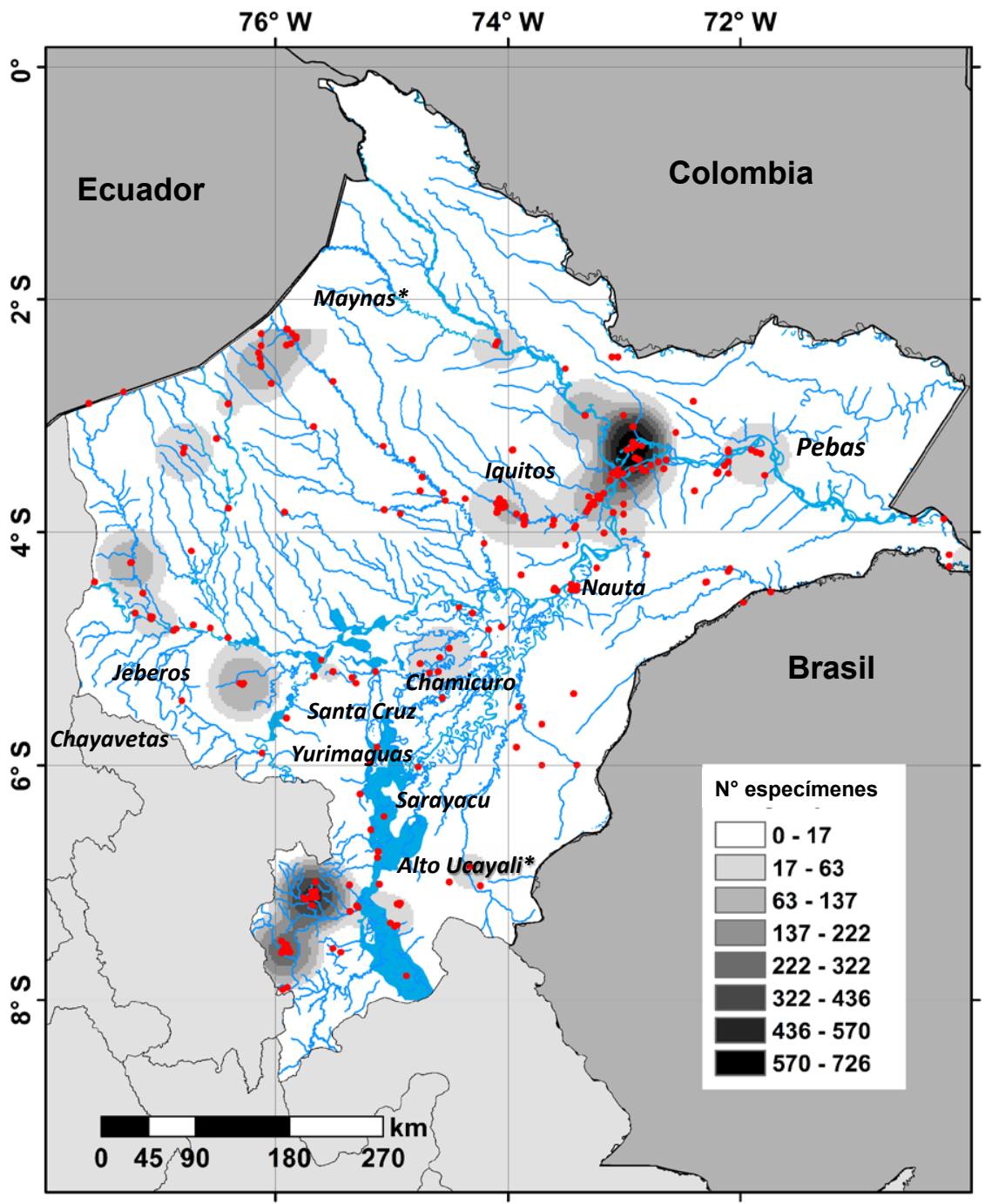

Figura 3. Localidades con colectas de aves en Loreto (círculos rojos); los colores del fondo muestran el número de especímenes colectados en diferentes áreas de Loreto (densidad Kernel de colecta).

Las aves de Loreto incluyen a 171 especies presentes en los apéndices de la convención CITES que regula y protege a especies silvestres amenazadas por el comercio internacional (Tabla 1), seis de éstas en el Apéndice I: Jabiru mycteria, Harpia harpyja, Falco peregrinus, Primolius couloni, Ara militaris y Ara macao. Otras 159 especies están incluidas en el Apéndice II y seis en el Apéndice III (Tabla 1). Por otro lado, 18 especies están incluidas en los apéndices de la CMS que protege a las especies migratorias (Tabla 1), dos de las cuales se encuentran en el Apéndice I: Calidris subruficollis y Setophaga cerulea. Ambas especies son migratorias del hemisferio norte, pero mientras que $C$. subruficollis es de hábitat acuático o de orilla, $S$. cerulea es de bosque. Esta última especie es una de las especies de parúlidos con mayor declinación de sus poblaciones reproductivas en Norteamérica y un paseriforme pequeño con uno de los viajes migratorios más largos (Welton et al. 2012, Delancey et al. 2020). C. subruficollis, por otro lado, es una especie de playero que se reproduce en las costas árticas de Norteamérica y Rusia y migra en el invierno boreal hasta Sudamérica (Lanctot et al. 2016) y ha sufrido una gran disminución de su población en el último siglo (Lounsberry et al. 2014).
Dentro de las especies de aves de Loreto, 13 son endémicas del Perú, dos picaflores Phaethornis koepckeae (Koepcke's Hermit), Heliodoxa branickii (Rufous-webbed Brilliant); un capito Capito wallacei (Scarlet-banded Barbet); tres hormigueritos Herpsilochmus parkeri (Ashthroated Antwren), Percnostola arenarum (Allpahuayo Antbird), Pithys castaneus (White-masked Antbird); un tapaculo Scytalopus femoralis (Rufous-vented Tapaculo); dos saltarines Lepidothrix coeruleocapilla (Ceruleancapped Manakin), Machaeropterus eckelberryi (Painted Manakin); un tororoi Grallaria carrikeri (Pale-billed Antpitta); un mosquerito Zimmerius villarejoi (Mishana Tyrannulet); una reinita Polioptila clementsi (Iquitos Gnatcatcher), y una tangara Ramphocelus melanogaster (Black-bellied Tanager).

Percnostola arenarum, Pithys castaneus y Polioptila clementsi son endémicas de Loreto. Las dos primeras tienen varias localidades de registro (principalmente, a orillas del Marañón, del Morona, del Nanay y en la Reserva Nacional Allpahuayo-Mishana), pero P. clementsi solo tiene registros en Allpahuayo-Mishana (endémica local). 


\section{Conclusiones}

Este trabajo registra 1040 especies de aves para Loreto, un número muy alto de especies considerando el número total de especies reportadas para Perú (representa el $55.3 \%$ del total), lo reportado en otras zonas de similares superficies en el Neotrópico (como el Matto Grosso do Sul en Brasil con 881 especies) y en otras regiones del mundo (como Alemania o Japón, 544 y 719 especies respectivamente). Considerando los Passeriformes, Loreto tiene el $52.2 \%$ del total de especies reportadas para la Amazonia en su totalidad (Fluck et al. 2020). La inclusión del área de Loreto en cinco provincias biogeográficas distintas (Morrone 2014) es una posible explicación de esta gran diversidad de aves, incluyendo algunas de relativamente amplia distribución que debieran ser estudiadas con herramientas moleculares en búsqueda de especies crípticas (Milá et al. 2012), existiendo en la actualidad escasa representación de secuencias de ADN de las aves de Loreto (Rojas et al. 2018).

La lista resultante de este trabajo tiene una alta confiabilidad debido al respaldo de cerca del $81 \%$ de las especies en especímenes de colecciones científicas y también porque apenas el $1.5 \%$ de las especies fueron incluidas solo por reportes de avistamientos realizados por ornitólogos. Si bien los registros de avistamientos realizados por aficionados (ciencia ciudadana) son una importante herramienta para estudios en ecología y a escalas mayores (Sullivan et al. 2014), es necesario tener mucho cuidado en el uso de esta información para listados de regiones de pequeña escala espacial, pues presentan generalmente muchos errores, que causan asunciones y sesgos peligrosos (Johnston et al. 2021). En especial son importantes, en escalas espaciales menores, los errores de "falsos positivos", es decir identificaciones erradas que reportan una especie en una localidad donde no se encuentra, los que incluso siendo pocos pueden generar amplios sesgos (Johnston et al. 2021, Miller et al. 2011).

La distribución de las colectas y registros de aves en Loreto, muestran que es necesario explorar áreas cercanas a los límites con Colombia y Brasil, principalmente, así como las zonas de mayores altitudes en las cordilleras que comparten con Amazonas y San Martín. Los estudios realizados dentro del programa de inventarios biológicos rápidos del Field Museum of Natural History, han demostrado no sólo la gran diversidad de especies de varios grupos taxonómicos, sino que han sido claves para la protección de extensas áreas de bosques, mostrando la importancia de Loreto en la conservación de la biodiversidad amazónica (Pitman et al. 2021). Nuestro análisis identifica que un importante porcentaje de las aves en alguna categoría de amenaza o endémicas tienen distribución restringida a áreas montanas o bosques de arena blanca, ambientes que tienen poca protección del Estado y presentan fuertes amenazas. Son necesarios estudios detallados de la distribución de las especies de aves, en general, y en particular de las especies amenazadas y endémicas, para asegurar una conservación efectiva de esta especialmente importante avifauna regional.

\section{Literatura citada}

Acosta Diaz A, Ayapi-Da-Silva J, Ocampo-Rodríguez M, Gálvez Carrillo H. 2019. Riqueza y características morfométricas de aves de sotobosque en Varillal Alto Seco de la Reserva Nacional Allpahuayo Mishana, Perú. Revista de Investigaciones Veterinarias del Perú 30(2):709720. https://doi.org/10.15381/rivep.v30i2.16074.

Alvarez Alonso J. 2010. Biodiversidad, conservación y turismo. In: Memoria Institucional 2010. Instituto de Investigaciones de la Amazonia Peruana. p. 60-62.

Álvarez Alonso J, Díaz Alván J, Shany N. 2012. Avifauna de la Reserva Nacional Allpahuayo Mishana, Loreto, Perú. Cotinga 34:61-84.

Angulo-Pérez NC, Armas-Silva JA, Gallardo D, Díaz J. 2018. Aves. In: Pérez-Peña PE, Ramos-Rodríguez MC, Díaz J, Zárate $\mathrm{R}$, Mejía $\mathrm{K}$, editors. Biodiversidad en las cuencas del Napo y Curaray, Perú. Instituto de Investigaciones de la Amazonia peruana. Imprenta Luanos EIRL. p. 99-126.

AMNH. 2020. American Museum of Natural History Vertebrate Zoology Collection Database. http://sci-web-001. amnh.org. Acceso 12/2020.

Armacost Junior JW. 2006. Birds of a palm-dominated terra firme forest: The contribution of habitat heterogeneity to regional avian diversity. Cotinga (25):33-37.

Armacost JW, Capparella AP. 2012. Use of mainland habitats by supposed river-island obligate birds along the Amazon River in Peru. Condor 114(1):56-61. https://doi. org/10.1525/cond.2012.100200.

Avibase. 2020. Avibase - The World Bird Database. https://avibase.bsc-eoc.org/.

Bartlett E. 1882. On some Mammals and Birds collected by Mr. J. Hauxwell in Eastern Peru. Proceedings of the Scientific Meetings of the Zoological Society of London. 373-374.

BHL. 2020. Biodiversity Heritage Library. https://www.biodiversitylibrary.org/.

Bicerra Canayo A. 2013. Aves Acuaticas y ribereñas en la zona baja y media del rio Samira, Loreto-Perú. Universidad Nacional de la Amazonia Peruana.

Brabourne, Lord, Chubb C. 1917. A List of the Birds of South America. London: R.H. Porter.

Burney CW, Brumfield RT. 2009. Ecology Predicts Levels of Genetic Differentiation in Neotropical Birds. American Naturalist 174(3):358-368. https://doi. org/10.1086/603613.

CITES. 2020. Checklist of CITES Species. http://checklist.cites. org. Acceso 12/2020.

CMS. 2020. Convention on the Conservation of Migratory Species of Wild Animals Species List. http://www.cms. int/en/species. Acceso 12/2020.

Decreto Supremo N 004-2014-MINAGRI. Decreto Supremo que aprueba la actualización de la lista de clasificación y categorización de las especies amenazadas de fauna silvestre. 08 de abril de 2014. El Peruano, Normas legales:520497-520504.

Delancey CD, Islam K, Kramer GR, MacDonald GJ, Sharp AR, Connare BM. 2020. Geolocators reveal migration routes, stopover sites, and nonbreeding dispersion in a population of Cerulean Warblers. Animal Migration 7(1):19-26. https://doi.org/10.1515/ami-20200003. 
Díaz-Alván J, Socolar JB, Álvarez Alonso J. 2017. The avifauna of the río Tigre basin, northern Perú. Ornitología Neotropical 28:11-21.

eBird. 2020. eBird: An online database of bird distribution and abundance. http://www.ebird.org. Acceso 12/2020.

Fluck IE, Cáceres N, Hendges CD, Brum M do N, Dambros CS. 2020. Climate and geographic distance are more influential than rivers on the beta diversity of passerine birds in Amazonia. Ecography (Cop). 43(6):860-868. https://doi.org/10.1111/ecog.04753.

Fong Rengifo EE, Mandujano Collantes JR, Velásquez Ruiz EM, Saravia Llaja KG. 2020. Primer registro documentado del Zorzal de Dorso Rojizo , Catharus fuscescens (Aves: Turdidae) para Loreto, Perú. Revista Peruana de Biología 27(4):529-532. http://dx.doi.org/10.15381/ rpb.v27i4.17726.

Foss C, Shaney N, Bicerra A. 2021. Bird List: Status based on expeditions from Tahuayo Lodge and Amaonia Research Center Peru Lodge. https://perujungle.com/ bird-list/.

García Bravo A, Barrio J. 2014. New distribution records of the Buff-fronted Owl Aegolius harrisii Cassin, 1849 (Aves: Strigidae) in Peru. Check List. 10(1):156-159. https:// doi.org/10.15560/10.1.156.

Garcia Solsol LA, Tuesta Cometivos GA, Ramírez Arévalo FF, Giardenelli A, Díaz Alván J. 2020. Primer registro documentado de la Cigüeña Maguari (Ciconia maguari Gmelin, 1789; Aves: Ciconiidae) en Loreto, Perú. Ciencia Amazónica 8(2):265-272. https://doi.org/http:// dx.doi.org/10.22386/ca.v8i2.302.

GBIF.org. 2020. GBIF Home Page. Available from: https://www. gbif.org [13 diciembre 2020].

Graves GR, Lane DF, O’Neill JP, Valqui T. 2011. A distinctive new subspecies of the Royal Sunangel (Aves: Trochiliformes; Heliangelus regalis) from the Cordillera Azul northern Peru. Zootaxa. 3002:52-58. https://doi. org/10.11646/zootaxa.3002.1.5.

Harvey MG, Aleixo A, Ribas CC, Brumfield RT. 2017. Habitat Association Predicts Genetic Diversity and Population Divergence in Amazonian Birds. American Naturalist 190(5):631-648. https://doi.org/10.1086/693856.

Harvey MG, Bravo GA, Claramunt S, Cuervo AM, Derryberry GE, Battilana J, Seeholzer GF, Shearer McKay J, O'Meara BC, Faircloth BC, et al. 2020. The evolution of a tropical biodiversity hotspot. Science (80- ). 370(6522):13431348. https://doi.org/10.1126/science.aaz6970.

Hastert E. 1918. Types of birds in the Tring Museum. Novit Zool. XXV:4-63.

iDigBio. 2020. Integrated Digitized Biocollections. http://www. idigbio.org. Acceso 12/2020.

INEI. 2017. Loreto; Compendio Estadístico 2017. Lima: Instituto Nacional de Estadística e Informática.

IUCN. 2020. The IUCN red list of threatened species, 2020 http://www.iucnredlist.org. Acceso 12/2020.

Johnston A, Hochachka WM, Strimas-Mackey ME, Ruiz Gutierrez V, Robinson OJ, Miller ET, Auer T, Kelling ST, Fink D. 2021. Analytical guidelines to increase the value of community science data: An example using eBird data to estimate species distributions. Diversity and Distribution (27):1265-1277. https://doi.org/10.1111/ ddi.13271.

Lanctot RB, Yezerinac S, Aldabe J, de Almeida JB, Castresana G, Brown S, Rocca P, Saalfeld ST, Fox JW. 2016. Lightlevel geolocation reveals migration patterns of the buff-breasted sandpiper. Wader Study. 123(1):29-43. https://doi.org/10.18194/ws.00032.

Lane DF, Kratter AW, Neill JP. 2017. A new species of manakin (Aves: Pipridae; Machaeropterus) from Peru with a taxonomic reassessment of the Striped Manakin (M. regulus) complex. Zootaxa. 4320(2):379-390. https:// doi.org/10.11646/zootaxa.4320.2.11.

Lane DF, Pequeño T, Flores Villar J. 2003. Aves. In: Perú: Yavarí. Rapid Biological Inventories Report 11. Chicago, Illinois.

Lane DF, Valqui T, Alvarez A J, Armenta J, Eckhardt K. 2006. The Rediscovery and Natural History of the Whitemasked Antbird (Pithys castaneus). Wilson Journal of Ornithology 118(1):13-22. https://doi.org/10.2173/ nb.whmant2.01.

Lecroy M. 2005. Type specimens of birds in the American museum of natural history part 6. Passeriformes: Prunellidae, Turdidae, Orthonychidae, Timaliidae, Paradoxornithidae, Picathartidae and Polioptilidae. Bulletin of the American Museum of Natural History 292:132. https://doi.org/10.1206/724.1.

Lecroy M. 2012. Type Specimens of Birds in the American Museum of Natural History Part 10. Passeriformes: Emberizidae: Emberizinae, Catamblyrhynchinae, Cardinalinae, Thraupinae, and Tersininae. Bulletin of the American Museum of Natural History 368:125.

LeCroy M, Sloss R. 2000. Type specimens of birds in the American Museum of Natural History. Part 3. Passeriformes: Eurylaimidae, Dendrocolaptidae, Furnariidae, Formicariidae, Conopophagidae, and Rhinocryptidae. Bulletin of the American Museum of Natural History (257):88. http://hdl.handle.net/2246/1607

Lounsberry ZT, Almeida JB, Lanctot RB, Liebezeit JR, Sandercock BK, Strum KM, Zack S, Wisely SM. 2014. Museum collections reveal that Buff-breasted Sandpipers (Calidris subruficollis) maintained mtDNA variability despite large population declines during the past 135 years. Conservation Genetics 15(5):1197-1208. https://doi.org/10.1007/s10592-014-0611-2.

Lüthi H. 2011. Birdwatching in Peru: 1963-2006. Revista Peruana de Biología 18(1):27-90. https://doi. org/10.15381/rpb.v18i1.170

Martínez AE, Robinson SK. 2016. Using foraging ecology to elucidate the role of species interactions in two contrasting mixed-species flock systems in northeastern Peru. Wilson Journal of Ornithology 128(2):378-390. https://doi.org/10.1676/wils-128-02-378-390.1.

Martínez AE, Zenil RT. 2012. Foraging guild influences dependence on heterospecific alarm calls in Amazonian bird flocks. Behavioral Ecology 23(3):544-550. https:// doi.org/10.1093/beheco/arr222.

Milá B, Tavares ES, Muñoz Saldaña A, Karubian J, Smith TB, Baker AJ. 2012. A Trans-Amazonian Screening of mtDNA Reveals Deep Intraspecific Divergence in Forest Birds and Suggests a Vast Underestimation of Species Diversity. PLoS ONE 7(7): e40541. https://doi. org/10.1371/journal.pone.0040541.

Miller DA, Nichols JD, McClintock BT, Campbell Grant EH, Bailey LL, Weir LA. 2011. Improving occupancy estimation when two types of observational error occur: non-detection and species misidentification. Ecology 92(7):1422-1428. https://doi.org/10.1890/101396.1 .

MINAM. 2015. Mapa de cobertura vegetal del Perú. Lima: Ministerio del Ambiente. 
Mlíkovský J. 2009. Types of birds in the collections of the Museum and Institute of Zoology, Polish Academy of Sciences, Warszawa, Poland. Part 4: Varia, adden$\mathrm{da}$ and conclusions. Journal of the National Museum (Prague), Natural History Series 178(5):17-180.

Moncrieff AE, Johnson O, Lane DF, Álvarez Alonso J, Balta K, Eckhardt K, Armenta J, Valqui T, Hernández F, Soto Huaira M, et al. 2019. Avifaunal surveys along the lower Huallaga River, Region of Loreto, Peru: New distributional records, collection of topotypes, and taxonomic implications. Wilson Journal of Ornithology 131(3):486-501. https://doi.org/10.1676/18-108.

Morrone JJ. 2014. Biogeographical regionalisation of the Neotropical region. Auckland: Magnolia Press-Zootaxa. https://doi.org/10.11646/zootaxa.3782.1.1.

Naranjo L, Salinas L, Quinteros K, Chiu A, Chota S, Talexio G, Tangoy H, Chimbo D, Macanilla D, Gómez L. 2016. Caracterización ornitológica del complejo lagunar Lagartococha. In: Usma J, Ortega C, Valenzuela S, Deza J, Rivas J, editors. Diversidad biológica y cultural del corredor trinacional de áreas protegidas La Paya - $\mathrm{Cu}$ yabeno - GUeppí Sekime. WWF Colombia.

NatureServe. 2020. NatureServe Explorer. https://www.natureserve.org. Acceso 12/2020.

NMNH. 2020. Smithsonian National Museum of Natural History Collection Records. http://collections.nmnh.si.edu. Acceso 12/2020.

O'Neill JP, Lane DF, Kratter AW, Capparella AP, Fox Joo C. 2000. A striking new species of barbet (Capitoninae: Capito) from the eastern Andes of Peru. Auk 117(3):569-577. https://doi.org/10.2307/4089583.

O'Neill JP, Lane DF, Naka LN. 2011. A cryptic new species of Thrush (Turdidae: Turdus) from Western Amazonia. Condor 113(4):869-880. https://doi.org/10.1525/ cond.2011.100244.

O’Shea BJ, Stotz DF, Saboya Del Castillo P, Ruelas Inzunza E. 2015. Aves. In: Perú: Tapiche-Blanco. Rapid Biological and Social Inventories Report 27. Chicago, Illinois: The Field Museum.

Pérez-Peña PE, Ramos-Rodríguez MC, Díaz-Alván J, ZárateGómez R, Mejía K. 2019. Biodiversidad en la Cuenca Alta del Putumayo, Perú. Iquitos, Perú: Instituto de Investigaciones de la Amazonia Peruana. https://repositorio.iiap.gob.pe/bitstream/20.500.12921/403/1/ Pérez_Libro_2019.pdf

Pitman N, Gagliardi Urrutia G, Jenkins C. 2013. La Biodiversidad de Loreto, Perú: El conocimiento actual de la diversidad de plantas y vertebrados terrestres. Center for International Environmental Law (CIEL).

Pitman NCA, Vriesendorp CF, Reyes DA, Moskovits DK, Kotlinski N, Smith RC, Thompson ME, Wali A, Benavides Matarazzo M, del Campo A, et al. 2021. Applied science facilities the large-scale expansion of protected areas in an Amazonian hot spot. Science Advances 7(31): eabe2998. https://doi.org/10.1126/sciadvabe2998.

Plenge MA. 2021. List of the birds of Peru / Lista de las aves del Perú. Unión de Ornitólogos del Perú. https://sites.google.com/site/boletinunop/checklist. Acceso 12/2021.

Pulido V, Salinas L, del Pino J, Arana C. 2021. Revisión del conocimiento actual y conservación de la lechuza de los arenales Athene cunicularia (Molina, 1782) en el Perú. Rev Peru Biol. 28(1): e19242. https://doi. org/10.15381/rpb.v28i1.19242.

Remsen JV, Areta JI, Bonaccorso E, Claramunt S, Jaramillo A, Lane DF, Pacheco JF, Robbins MB, Stiles FG, Zimmer
KJ. 2020. A classification of the bird species of South America. American Ornithological Society. http:// www.museum.lsu.edu/ Remsen/SACCCountryLists. htm. Acceso 12/2020.

Remsen, J V., Hyde MA, Chapman A. 1993. The Diets of Neotropical Trogons, Motmots, Barbets and Toucans. Condor 95(1):178-192. https://doi.org/10.2307/1369399.

Remsen J V., Parker III TA. 1983. Contribution of RiverCreated Habitats to Bird Species Richness in Amazonia. Biotropica 15(3):223-231. https://doi. org $/ 10.2307 / 2387833$.

Rojas RR, Castro JC, García-Dávila C. 2018. Situación actual de la base de datos genéticos de vertebrados de la región Loreto, Perú. Folia Amazónica 27(2):151-162. https:// doi.org/10.24841/fa.v27i2.442.

Roselaar CS. 2003. An inventory of major European bird collections. Bull Ornithol Club. 123:253-337.

Rosenberg GH. 1990. Habitat Specialization and Foraging Behavior by Birds of Amazonian River Islands in Northeastern Peru. Condor. 92:427-443. https://doi. org/10.2307/1368240.

Ruelas Inzunza E, Zeppilli Tizón R, Stos. 2014. Aves/Birds. In: Perú: Cerros de Kampankis. Rapid Biological and Social Inventories Report 24.

Ruiz-Ramos SA, Angulo-Perez NC, Del Castillo PS, Gaviria Celis JL, Díaz Alván J. 2020. Registros documentados del Corocoro Escarlata (Eudocimus ruber Linnaeus, 1758; Aves: Threskiornithidae) en Loreto, Perú. Ciencia Amazónica 8(1):123-130. https://doi.org/10.22386/ ca.v8i1.285.

Salazar E, Mattos J, Díaz J, Ferreyra F, Piana R, Balta K. 2003. Composición de especies de aves en hábitats de la Zona Reservada Allpahuayo - Mishana y Colinas de la formación Nauta, Loreto, Perú. Folia Amazónica 14(1):125-132. http://www.iiap.org.pe/Upload/Publicacion/PUBL664.pdf.

Schmitt F, Sané R, Thibault M, Vásquez G. 2017. New locality for White-masked Antbird Pithys castaneus and other avian range extensions for dpto. Loreto, Peru. Cotinga. 39:2-11.

Schulenberg TS, Albujar C, Rojas JI. 2006. Aves. In: Peru: Sierra del Divisor. Rapid Biological Inventories Report 17. Chicago, Illinois: The Field Museum.

Schulenberg TS, Stotz DF, Lane DF, O'Neill JP, Parker III TA. 2007. Birds of Peru. Princeton University Press, Princeton, New Jersey, USA. 664 pp.

Schulenberg TS, Lane DF, O'Neill JP, Stotz DF. 2008. Field identification of juvenile Empidonomus tyrants. Neotropical Bird 3:31-36.

Schulenberg TS, O’Neill JP, Lane DF, Valqui T, Albújar C. 2001. Aves. In: Perú: Biabo Cordillera Azul. Rapid Biological Inventories Report 2. Chicago, Illinois.

Schulenberg TS, Stotz DF, Lane DF, O’Neill JP, Parker III TA. 2007. Birds of Peru. Revised an. Princeton, New Jersey: Princeton University Press. http://library1.nida. ac.th/termpaper6/sd/2554/19755.pdf.

Sclater PL. 1857b. On a collection of birds transmitted by Mr. H. W. Bates from the Upper Amazon. Proceedings of the Zoological Society of London. 25: 261-268. https:// doi.org/10.1111/j.1096-3642.1857.tb01238.x.

Sclater PL, \& Salvin O. 1867. List of birds collected at Pebas, upper Amazons, by Mr. John Hauxwell, with notes and descriptions of new species. Proceedings of the Zoological Society of London 1867:977-981. 
Shahuano Tello N, Stojan-Dolar M, Heymann EW. 2008. A sight and video record of the oilbird, Steatornis caripensis, in Peruvian lowland Amazonia. Journal of Ornithology149:267-269. https://doi.org/10.1007/s10336007-0266-6.

Sharpe RB. 1874. Catalogue of the birds in the British Museum. London: British Museum (Natural History) Department of Zoology, Printed by order of the Trustees.

Socolar JB, Díaz-Alván J, Saboya Del Castillo P, Pomara LY, O'Shea BJ, Cubas Poclin S, Stotz D, Schmitt F, Graham D, Carnes BH, et al. 2018. Noteworthy bird records from northeastern Peru reveal connectivity and isolation in the western Amazonian avifauna. Wilson Journal of Ornithology 130(1):94-111. doi:10.1676/16-082.1.

Sullivan BL, Aycrigg JL, Barry JH, Bonney RE, Bruns N, Cooper CB, Damoulas T, Dhondt AA, Dietterich T, Farnsworth A, et al. 2014. The eBird enterprise: An integrated approach to development and application of citizen science. Biological conservation 169(2014):31-40. https://doi.org/10.1016/j.biocon.2013.11.003

Taczanowski L. 1884a. Ornithologie du Pérou. Vol. 1. Rennes: Typographie Oberthur.

Taczanowski L. 1884b. Ornithologie du Pérou. Vol. 2. Rennes: Typographie Oberthur.

Taczanowski L. 1886. Ornithologie du Pérou. Vol. 3. Rennes: Typographie Oberthur.

Ter Steege H, Pitman NCA, Sabatier D, Baraloto C, Salomão RP, Guevara JE, Phillips OL, Castilho C V., Magnusson WE, Molino JF, et al. 2013. Hyperdominance in the Amazonian tree flora. Science (80- ). 342(6156). https://doi. org/10.1126/science.1243092.

Stotz DF, Díaz-Alván J. 2007. Aves. Perú Nanay-Mazán-Arabela Rapid Biological Inventories Report 18.

Stotz DF, Díaz-Alván J. 2010. Aves. In: Perú: Maijuna. Rapid Biological and Social Inventories Report 22. Chicago, Illinois.

Stotz DF, Pequeño T. 2006. Aves. In: Perú: Matsés. Rapid Biological Inventories Report 16. Chicago, Illinois.

Stotz DF, Ruelas Inzunza E. 2013. Aves. In: Perú: Ere-CampuyaAlgodón. Rapid Biological and Social Inventories Report 25. Chicago.

Stotz DF, Saboya Del Castillo P, Laverde-R 0. 2016. Aves. In: Perú: Medio Putumayo-Algodón. Rapid Biological and Social Inventories Report 28. Chicago, Illinois.

Torres-Vásquez MI, Ungvari Martin J, Salo M. 2018. Muda Y Reproducción En Aves De Sotobosque De La Reserva Nacional Allpahuayo Mishana, Loreto, Perú. Ornitología Neotropical 29:229-239.

Ugarte M, Lavalle Valdivia M. 2018. Primer registro documentado del Gorrión de Collar Rufo Zonotrichia capensis (Aves: Emberizidae) en la Amazonia Peruana. Revista Peruana de Biología 25(2):175-178. https://doi. org/10.15381/rpb.v25i2.14077.

Ungvari Martin J. 2017. Connecticut Warbler (Oporornis agilis) in a Peruvian White Sand Forest Habitat. Boletín UNOP. 12(1):6-8.

Ungvari Martin J, Heckscher CM, Hobson KA. 2016. Interannual site fidelity and breeding origins of Gray-cheeked Thrushes in white sand forests of the Peruvian Amazon. Journal of Field Ornithology 87(1):55-64. https://doi.org/10.1111/jofo.12133.

van den Hoek Ostende L, Dekker R, Keijl G. 1997. Type-specimens of birds in the National Museum of Natural His- tory, Leiden Part 1. Non-Passerines. NNM Tech Bull. 1(30.xii):1-248.

Vásquez-Arévalo FA, Zárate-Gómez R, Socolar JB, Díaz-Alván J, Pérez-Peña PE. 2020. First record of the gray-legged tinamou, Crypturellus duidae, and other poor-soil specialist birds from peatlands in the Putumayo River basin, Loreto, Peru. Acta Amazonica 50(2):155-158. https://doi.org/10.1590/1809-4392201901531.

VertNet. 2020. VertNet. http://www.vertnet.org. Acceso $12 / 2020$.

Welton MJ, Anderson DL, Colorado GJ, Hamel PB, Calderón D. 2012. Spring migration stopover by Cerulean Warblers in northern Middle America. Ornitología Neotropical 23:285-302.

Whitney BM, Alvarez Alonso J. 2005. A new species of gnatcatcher from white-sand forests of northern Amazonian Peru with revision of the Polioptila guianensis complex. The Wilson Bulletin 117(2):113-127. https:// doi.org/10.1676/04-064.

Whitney BM, Cohn-Haft M. 2013. Fifteen new species of Amazonian birds. Handbook of the Birds of the World Spec Vol.(January 2013):225-239.

Wiley RH, Álvarez Alonso J, Díaz Alván J. 2014. Aves de Loreto Bajo (60-250 msnm). :1-10. http://rhwiley.bio.unc. edu/pdfs/AvesLoretoBajo202011.pdf.

Zimmer JT. 1924. Studies on Peruvian Birds. No. XL. Notes on the Genus Veniliornis. American Museum Novitates 1159:1-4. http://hdl.handle.net/2246/3222.

Zimmer JT. 1937. Studies on Peruvian Birds $\mathrm{N}^{\circ} 27$. Notes on the genera Muscivora, Tyrannus, Empidonomus, and Sirystes, with further notes on Knipolegus. American Museum Novitates 962:1-28.

\section{Agradecimientos / Acknowledgments:}

Agradecemos a Wildlife Conservation Society - Perú por el soporte principal para el presente estudio. Un especial agradecimiento a Juan Diaz por compartir su apreciación sobre algunos de los registros más controversiales de aves de Loreto. Gracias a Daniel Cossios por la invitación para este número especial. Agradecernos a Mario Pezua, Mónica Paredes, Daniel Muñoz y Juan Francisco Esteves por la ayuda en la recopilación de información de las bases de datos de colecciones científicas.

\section{Conflicto de intereses / Competing interests:}

CA es Editor en la RevPeruBiol, no intervino en el proceso editorial. Los autores no incurren en conflictos de intereses.

\section{Rol de los autores / Authors Roles:}

Debe decir: "LS y CA: conceptualización. LS y AA: validación y curación de los datos. LS, AA y CA: investigación, análisis formal, escritura, redacción y visualización del manuscrito.

\section{Fuentes de financiamiento / Funding:}

Wildlife Conservation Society - Perú, VRIP-UNMSM (Proyecto 161001161), FONDECYT (Proyecto 75357/Contrato 039-2021-FONDECYT).

\section{Aspectos éticos / legales; Ethics / legals:}

Los autores declaran no haber incurrido en ningún aspecto antiético. La información de campo utilizada se obtuvo bajo los permisos de investigación RD 294-2015-SERFOR- DGGSPFFS y RD 025-2008-INRENA-IFFS-DCB, Ministerio de Agricultura y Riego, Dirección General Forestal y de Fauna Silvestre. 
SALINAS ET AL.

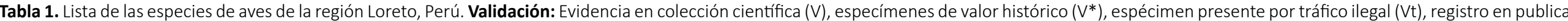

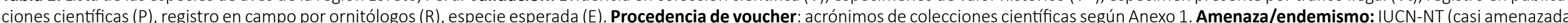

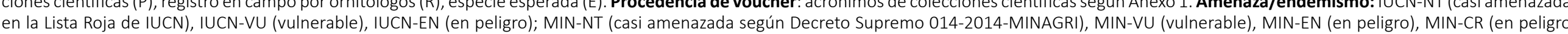



\begin{tabular}{|c|c|c|c|c|}
\hline Nombre científico & Nombre en inglés & Validación & Procedencia de Voucher & Amenaza/endemismo \\
\hline \multicolumn{5}{|l|}{ Orden Tinamiformes: Familia Tinamidae } \\
\hline Tinamus tao Temminck, 1815 & Gray Tinamou & VPR & FMNH, LSUMZ, MUSM & IUCN-VU \\
\hline Tinamus major (Gmelin, JF, 1789) & Great Tinamou & VPR & CLO, FMNH, KU, LSUMZ, MCZ, MUSM & IUCN-NT \\
\hline Tinamus guttatus von Pelzeln, 1863 & White-throated Tinamou & $\mathrm{V} * \mathrm{PR}$ & CLO, FMNH, LSUMZ, MUSM, UF-FLMNH & IUCN-NT \\
\hline Crypturellus cinereus (Gmelin, JF, 1789) & Cinereous Tinamou & $\mathrm{V} * \mathrm{PR}$ & CLO, FMNH, LSUMZ, MUSM, RMNH & \\
\hline Crypturellus soui (Hermann, 1783) & Little Tinamou & VPR & CLO, LSUMZ, MUSM & \\
\hline Crypturellus obsoletus (Temminck, 1815) & Brown Tinamou & $\mathrm{VP}$ & CLO & \\
\hline Crypturellus undulatus (Temminck, 1815) & Undulated Tinamou & VPR & CLO, LSUMZ, MCZ, MUSM, UF-FLMNH & \\
\hline Crypturellus strigulosus (Temminck, 1815) & Brazilian Tinamou & $\mathrm{P}$ & & \\
\hline Crypturellus duidae Zimmer, JT, 1938 & Gray-legged Tinamou & $\mathrm{P}$ & & IUCN-NT, MIN-VU \\
\hline Crypturellus variegatus (Gmelin, JF, 1789) & Variegated Tinamou & VPR & FMNH, LSUMZ, MCZ, MUSM, WFVZ & \\
\hline Crypturellus bartletti (Sclater \& Salvin, 1873) & Bartlett's Tinamou & $\mathrm{V}$ PRR & FMNH, LSUMZ, MUSM, UF-FLMNH, USNM & \\
\hline Crypturellus casiquiare (Chapman, 1929) & Barred Tinamou & $\mathrm{P}$ & & MIN-VU \\
\hline \multicolumn{5}{|l|}{ Orden Anseriformes: Familia Anhimidae } \\
\hline Anhima cornuta (Linnaeus, 1766) & Horned Screamer & $\mathrm{V} * \mathrm{PR}$ & AMNH, CLO, FMNH, LSUMZ, MCZ, MUSM, UF-FLMNH & \\
\hline \multicolumn{5}{|l|}{ Orden Anseriformes: Familia Anatidae } \\
\hline Dendrocygna bicolor (Vieillot, 1816) & Fulvous Whistling-Duck & VP & MUSM & CITES-III, CMS-II \\
\hline Dendrocygna viduata (Linnaeus, 1766) & White-faced Whistling-Duck & $\mathrm{P}$ & & CMS-II \\
\hline Dendrocygna autumnalis (Linnaeus, 1758) & Black-bellied Whistling-Duck & VPR & MUSM & CITES-III \\
\hline Oressochen jubatus (von Spix, 1825) & Orinoco Goose & PR & & IUCN-NT, MIN-VU \\
\hline Cairina moschata (Linnaeus, 1758) & Muscovy Duck & $\mathrm{V} * \mathrm{PR}$ & LSUMZ & \\
\hline Sarkidiornis sylvicola von Ihering, $\mathrm{H} \&$ von Ihering, $\mathrm{R}, 1907$ & American Comb-Duck & $\mathrm{R}$ & & \\
\hline Amazonetta brasiliensis (Gmelin, JF, 1789) & Brazilian Teal & $\mathrm{P}$ & & \\
\hline Spatula discors (Linnaeus, 1766) & Blue-winged Teal & PR & & \\
\hline Anas bahamensis Linnaeus, 1758 & White-cheeked Pintail & $R$ & & \\
\hline Anas flavirostris Vieillot, 1816 & Yellow-billed Teal & $R$ & & \\
\hline Nomonyx dominicus (Linnaeus, 1766) & Masked Duck & PR & $\therefore$ & \\
\hline \multicolumn{5}{|l|}{ Orden Galliformes: Familia Cracidae } \\
\hline Chamaepetes goudotii (Lesson, 1828) & Sickle-winged Guan & VR & LSUMZ & \\
\hline Penelope jacquacu von Spix, 1825 & Spix's Guan & $\mathrm{V}$ PR & CLO, FMNH, KU, LSUMZ, MUSM & \\
\hline Pipile cumanensis (von Jacquin, 1784) & Blue-throated Piping-Guan & VPR & FMNH, LSUMZ, MCZ, MUSM, MVZ & MIN-NT \\
\hline Aburria aburri (Lesson, 1828) & Wattled Guan & VP & CLO, LSUMZ, MUSM & IUCN-NT, MIN-VU \\
\hline Ortalis guttata (von Spix, 1825) & Speckled Chachalaca & V*PR & CLO, FMNH, LSUMZ, MUSM, MVZ, UF-FLMNH & \\
\hline Nothocrax urumutum (von Spix, 1825) & Nocturnal Curassow & $\mathrm{V*PR}$ & CLO, KU, LSUMZ, UF-FLMNH & \\
\hline Crax globulosa von Spix, 1825 & Wattled Curassow & $\mathrm{V*P}$ & UMMZ & IUCN-EN, MIN-CR, CITES-III \\
\hline Mitu salvini Reinhardt, 1879 & Salvin's Curassow & VPR & CLO & MIN-VU \\
\hline
\end{tabular}


LAS AVES DEL DEPARTAMENTO DE LORETO

\begin{tabular}{|c|c|c|c|c|}
\hline Nombre científico & Nombre en inglés & Validación & Procedencia de Voucher & Amenaza/endemismo \\
\hline Mitu tuberosum (von Spix, 1825) & Razor-billed Curassow & $\mathrm{V}$ PR & LSUMZ, MUSM & MIN-NT \\
\hline \multicolumn{5}{|l|}{ Orden Galliformes: Familia Odontophoridae } \\
\hline Odontophorus gujanensis (Gmelin, JF, 1789) & Marbled Wood-Quail & VPR & CLO, FMNH, LSUMZ, MUSM, WFVZ, YPM & IUCN-NT \\
\hline Odontophorus speciosus von Tschudi, 1843 & Rufous-breasted Wood-Quail & VPR & LSUMZ, MUSM & IUCN-NT \\
\hline Odontophorus stellatus (Gould, 1843) & Starred Wood-Quail & $\mathrm{V} * \mathrm{P}$ & FMNH, LSUMZ, MUSM & \\
\hline \multicolumn{5}{|l|}{ Orden Podicipediformes: Familia Podicipedidae } \\
\hline Tachybaptus dominicus (Linnaeus, 1766) & Least Grebe & $\mathrm{P}$ & & \\
\hline \multicolumn{5}{|l|}{ Orden Columbiformes: Familia Columbidae } \\
\hline Columba livia Gmelin, JF, 1789 & Rock Pigeon & $P R$ & & \\
\hline Patagioenas speciosa (Gmelin, JF, 1789) & Scaled Pigeon & $\mathrm{V} * \mathrm{PR}$ & MUSM & \\
\hline Patagioenas fasciata (Say, 1822) & Band-tailed Pigeon & $\mathrm{E}$ & & \\
\hline Patagioenas cayennensis (Bonnaterre, 1792) & Pale-vented Pigeon & $\mathrm{V} * \mathrm{PR}$ & CLO, FMNH & \\
\hline Patagioenas plumbea (Vieillot, 1818) & Plumbeous Pigeon & VPR & CLO, FMNH, LSUMZ, MCZ, MUSM, UF-FLMNH & \\
\hline Patagioenas subvinacea (Lawrence, 1868) & Ruddy Pigeon & VPR & CLO, FMNH, LSUMZ, MCZ, MUSM & IUCN-VU \\
\hline Geotrygon saphirina Bonaparte, 1855 & Sapphire Quail-Dove & $\mathrm{VP}$ & FMNH, LSUMZ, MNHN, MUSM, YPM & \\
\hline Geotrygon montana (Linnaeus, 1758) & Ruddy Quail-Dove & V*PR & CLO, FMNH, KU, LSUMZ, MUSM, UMMZ & \\
\hline Leptotila verreauxi Bonaparte, 1855 & White-tipped Dove & VPR & CLO, MUSM & \\
\hline Leptotila rufaxilla (Richard \& Bernard, 1792) & Gray-fronted Dove & $\mathrm{V*PR}$ & CLO, FMNH, KU, LSUMZ, MCZ, MUSM & \\
\hline Zentrygon frenata (von Tschudi, 1843) & White-throated Quail-Dove & VPR & LSUMZ, MUSM & \\
\hline Zenaida auriculata (Des Murs, 1847) & Eared Dove & $\mathrm{P}$ & & \\
\hline Claravis pretiosa (Ferrari-Pérez, 1886) & Blue Ground Dove & $\mathrm{V} * \mathrm{PR}$ & LSUMZ & \\
\hline Columbina passerina (Linnaeus, 1758) & Common Ground Dove & $\mathrm{PE}$ & & \\
\hline Columbina minuta (Linnaeus, 1766) & Plain-breasted Ground Dove & $\mathrm{VP}$ & LSUMZ, MUSM & \\
\hline Columbina talpacoti (Temminck, 1810) & Ruddy Ground Dove & VPR & CLO, CM, FMNH, LSUMZ, MUSM, YPM & \\
\hline Columbina picui (Temminck, 1813) & Picui Ground Dove & $R$ & & \\
\hline \multicolumn{5}{|l|}{ Orden Cuculiformes: Familia Cuculidae } \\
\hline Crotophaga major Gmelin, JF, 1788 & Greater Ani & $\mathrm{V}$ PRR & CLO, CM, FMNH, LSUMZ, MCZ, MUSM, Unicamp-FNJV & \\
\hline Crotophaga ani Linnaeus, 1758 & Smooth-billed Ani & $\mathrm{V} * \mathrm{PR}$ & CLO, CM, FMNH, KU, LSUMZ, MCZ, MUSM, NHMO & \\
\hline Tapera naevia (Linnaeus, 1766) & Striped Cuckoo & $\mathrm{VP}$ & CLO & \\
\hline Dromococcyx phasianellus (von Spix, 1824) & Pheasant Cuckoo & VPR & CLO & \\
\hline Dromococcyx pavoninus von Pelzeln, 1870 & Pavonine Cuckoo & VP & MUSM, UF-FLMNH & \\
\hline Neomorphus geoffroyi (Temminck, 1820) & Rufous-vented Ground-Cuckoo & $\mathrm{VP}$ & LSUMZ & IUCN-VU \\
\hline Neomorphus pucheranii (Deville, 1851) & Red-billed Ground-Cuckoo & $\mathrm{V} * \mathrm{P}$ & CLO, FMNH, LSUMZ, YPM & \\
\hline Coccycua minuta (Vieillot, 1817) & Little Cuckoo & V*PR & CLO, FMNH, LSUMZ & \\
\hline Coccycua cinerea (Vieillot, 1817) & Ash-colored Cuckoo & $P$ & & \\
\hline Piaya cayana (Linnaeus, 1766) & Squirrel Cuckoo & $\mathrm{V} * \mathrm{PR}$ & CLO, CM, FMNH, LSUMZ, MNHN, MUSM, NHMO, UF-FLMNH, USNM & \\
\hline Piaya melanogaster (Vieillot, 1817) & Black-bellied Cuckoo & V*PR & CLO, FMNH, LSUMZ, MUSM, UMMZ & \\
\hline Coccyzus melacoryphus Vieillot, 1817 & Dark-billed Cuckoo & V*PR & CLO, FMNH, LSUMZ, UMMZ & \\
\hline Coccyzus americanus (Linnaeus, 1758) & Yellow-billed Cuckoo & $P R$ & & \\
\hline Coccyzus erythropthalmus (Wilson, A, 1811) & Black-billed Cuckoo & $P R$ & & \\
\hline
\end{tabular}


SALINAS ET AL.

\begin{tabular}{|c|c|c|c|c|}
\hline Nombre científico & Nombre en inglés & Validación & Procedencia de Voucher & Amenaza/endemismo \\
\hline \multicolumn{5}{|l|}{ Orden Steatornithiformes: Familia Steatornithidae } \\
\hline Steatornis caripensis von Humboldt, 1817 & Oilbird & VP & FMNH, LSUMZ, MUSM & \\
\hline \multicolumn{5}{|l|}{ Orden Nyctibiiformes: Familia Nyctibiidae } \\
\hline Nyctibius grandis (Gmelin, JF, 1789) & Great Potoo & VPR & CLO, KU, LSUMZ, MUSM, UF-FLMNH & \\
\hline Nyctibius aethereus (zu Wied-Neuwied, 1820) & Long-tailed Potoo & V*PR & CLO, LSUMZ, MUSM & \\
\hline Nyctibius griseus (Gmelin, JF, 1789) & Common Potoo & V*PR & CLO, FMNH, LSUMZ, MUSM, UF-FLMNH, UMMZ & \\
\hline Nyctibius leucopterus (zu Wied-Neuwied, 1821) & White-winged Potoo & $P$ & & MIN-VU \\
\hline Nyctibius bracteatus Gould, 1846 & Rufous Potoo & V*PR & LSUMZ & \\
\hline \multicolumn{5}{|l|}{ Orden Caprimulgiformes: Familia Caprimulgidae } \\
\hline Chordeiles nacunda (Vieillot, 1817) & Nacunda Nighthawk & V*P & DMNH & \\
\hline Chordeiles rupestris (von Spix, 1825) & Sand-colored Nighthawk & $\mathrm{V} * \mathrm{PR}$ & CLO, DMNH, FMNH, LSUMZ, MCZ, MUSM, UMMZ, USNM & \\
\hline Chordeiles minor (Forster, JR, 1771) & Common Nighthawk & PR & & \\
\hline Lurocalis semitorquatus (Gmelin, JF, 1789) & Short-tailed Nighthawk & VPR & CLO, LSUMZ & \\
\hline Nyctiprogne leucopyga (von Spix, 1825) & Band-tailed Nighthawk & $\mathrm{VP}$ & LSUMZ, MUSM & \\
\hline Nyctipolus nigrescens (Cabanis, 1849) & Blackish Nightjar & VPR & FMNH, LSUMZ, MUSM & \\
\hline Nyctidromus albicollis (Gmelin, JF, 1789) & Common Pauraque & $V * P R$ & CLO, FMNH, KU, LSUMZ, MCZ, MUSM, NHMO, UF-FLMNH, WFVZ & \\
\hline Setopagis parvula (Gould, 1837) & Little Nightjar & V*PR & UF-FLMNH & \\
\hline Hydropsalis maculicaudus (Lawrence, 1862) & Spot-tailed Nightjar & $P$ & & \\
\hline Hydropsalis climacocerca (von Tschudi, 1844) & Ladder-tailed Nightjar & V*PR & CLO, LSUMZ, MCZ, MUSM & \\
\hline Hydropsalis torquata (Gmelin, JF, 1789) & Scissor-tailed Nightjar & P & & \\
\hline Nyctiphrynus ocellatus (von Tschudi, 1844) & Ocellated Poorwill & VPR & LSUMZ, MUSM & \\
\hline Antrostomus sericocaudatus Cassin, 1849 & Silky-tailed Nightjar & VP & LSUMZ & \\
\hline \multicolumn{5}{|l|}{ Orden Apodiformes: Familia Apodidae } \\
\hline Cypseloides cryptus Zimmer, JT, 1945 & White-chinned Swift & $\mathrm{P}$ & & \\
\hline Cypseloides lemosi Eisenmann \& Lehmann, 1962 & White-chested Swift & $P$ & & \\
\hline Streptoprocne rutila (Vieillot, 1817) & Chestnut-collared Swift & $\mathrm{P}$ & & \\
\hline Streptoprocne zonaris (Shaw, 1796) & White-collared Swift & VPR & CLO & \\
\hline Chaetura spinicaudus (Temminck, 1839) & Band-rumped Swift & $P E$ & & \\
\hline Chaetura cinereiventris Sclater, PL, 1862 & Gray-rumped Swift & $V * P R$ & CLO, MUSM & \\
\hline Chaetura egregia Todd, 1916 & Pale-rumped Swift & VPR & CLO, LSUMZ, MUSM & \\
\hline Chaetura pelagica (Linnaeus, 1758) & Chimney Swift & $P$ & & IUCN-VU \\
\hline Chaetura chapmani Hellmayr, 1907 & Chapman's Swift & VPR & MUSM & \\
\hline Chaetura meridionalis Hellmayr, 1907 & Sick's Swift & PE & & \\
\hline Chaetura brachyura (Jardine, 1846) & Short-tailed Swift & $V * P R$ & CLO, LSUMZ, MUSM & \\
\hline Aeronautes montivagus (d'Orbigny \& Lafresnaye, 1837) & White-tipped Swift & VP & CLO, LSUMZ, MUSM & \\
\hline Tachornis squamata (Cassin, 1853) & Fork-tailed Palm-Swift & $V * P R$ & LSUMZ, MUSM & \\
\hline Panyptila cayennensis (Gmelin, JF, 1789) & Lesser Swallow-tailed Swift & $P R$ & & \\
\hline \multicolumn{5}{|l|}{ Orden Apodiformes: Familia Trochilidae } \\
\hline Topaza pyra (Gould, 1846) & Fiery Topaz & VPR & LSUMZ, MUSM & CITES-II \\
\hline Florisuga mellivora (Linnaeus, 1758) & White-necked Jacobin & V*PR & FMNH, LSUMZ, MUSM, UMMZ & CITES-II \\
\hline
\end{tabular}


LAS AVES DEL DEPARTAMENTO DE LORETO

\begin{tabular}{|c|c|c|c|c|}
\hline Nombre científico & Nombre en inglés & Validación & Procedencia de Voucher & Amenaza/endemismo \\
\hline Eutoxeres aquila (Bourcier, 1847) & White-tipped Sicklebill & $\mathrm{VP}$ & CLO, LSUMZ, MUSM, UMMZ & CITES-II \\
\hline Eutoxeres condamini (Bourcier, 1851) & Buff-tailed Sicklebill & $V * P R$ & KU, LSUMZ, MUSM & CITES-II \\
\hline Glaucis hirsutus (Gmelin, JF, 1788) & Rufous-breasted Hermit & $\mathrm{V*PR}$ & CM, FMNH, KU, LSUMZ, MUSM, TCWC, UMMZ, USNM & CITES-II \\
\hline Threnetes leucurus (Linnaeus, 1766) & Pale-tailed Barbthroat & $\mathrm{V} * \mathrm{PR}$ & CM, FMNH, KU, LSUMZ, MUSM & CITES-II \\
\hline Phaethornis atrimentalis Lawrence, 1858 & Black-throated Hermit & VPR & CLO, FMNH, LSUMZ, MUSM, WFVZ & CITES-II \\
\hline Phaethornis griseogularis Gould, 1851 & Gray-chinned Hermit & VPR & LSUMZ, MUSM & CITES-II \\
\hline Phaethornis ruber (Linnaeus, 1758) & Reddish Hermit & $\mathrm{V} * \mathrm{PR}$ & FMNH, LSUMZ, MUSM, WFVZ & CITES-II \\
\hline Phaethornis hispidus (Gould, 1846) & White-bearded Hermit & $V * P R$ & CLO, CM, FMNH, LSUMZ, MUSM, UMMZ & CITES-II \\
\hline Phaethornis guy (Lesson, 1833) & Green Hermit & $\mathrm{VP}$ & LSUMZ, MUSM, UF-FLMNH, UWBM & CITES-II \\
\hline Phaethornis koepckeae Weske \& Terborgh, 1977 & Koepcke's Hermit & $P R$ & & IUUCN-NT, MIIN-NTT, CITTES-III, \\
\hline Phaethornis philippii (Bourcier, 1847) & Needle-billed Hermit & VPR & FMNH, LSUMZ, MUSM & CITES-II \\
\hline Phaethornis bourcieri (Lesson, 1832) & Straight-billed Hermit & $V * P R$ & KU, FMNH, LSUMZ, MUSM, TCWC, UF-FLMNH & CITES-II \\
\hline Phaethornis malaris (Nordmann, 1835) & Great-billed Hermit & VPR & 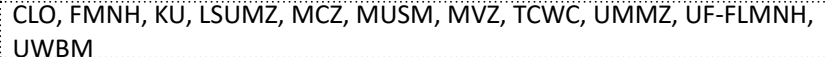 & CITES-II \\
\hline Doryfera ludovicae (Bourcier \& Mulsant, 1847) & Green-fronted Lancebill & $P R$ & & CITES-II \\
\hline Doryfera johannae (Bourcier, 1847) & Blue-fronted Lancebill & $\mathrm{VP}$ & LSUMZ, MUSM, UWBM & CITES-II \\
\hline Colibri delphinae (Lesson, 1839) & Brown Violetear & $\mathrm{P}$ & & CITES-II \\
\hline Colibri cyanotus (Bourcier, 1843) & Lesser Violetear & R & & CITES-II \\
\hline Colibri coruscans (Gould, 1846) & Sparkling Violetear & $\mathrm{PE}$ & & CITES-II \\
\hline Heliothryx auritus (Gmelin, JF, 1788) & Black-eared Fairy & VPR & LSUMZ & CITES-II \\
\hline Polytmus theresiae (Da Silva Maia, 1843) & Green-tailed Goldenthroat & VPR & LSUMZ, MUSM & CITES-II \\
\hline Avocettula recurvirostris (Swainson, 1822) & Fiery-tailed Awlbill & PR & & CITES-II \\
\hline Anthracothorax nigricollis (Vieillot, 1817) & Black-throated Mango & VPR & CM, LSUMZ, MUSM, UMMZ, USNM & CITES-II \\
\hline Heliangelus regalis Fitzpatrick, Willard \& Terborgh, 1979 & Royal Sunangel & $\mathrm{VP}$ & LSUMZ & IUCN-EN, MIN-VU, CITES-II \\
\hline Discosura popelairii (Du Bus, 1846) & Wire-crested Thorntail & VPR & CLO & IUCN-NT, CITES-II \\
\hline Discosura langsdorffi (Temminck, 1821) & Black-bellied Thorntail & $\mathrm{V} * \mathrm{PR}$ & FMNH, LSUMZ, UMMZ, USNM & CITES-II \\
\hline Lophornis delattrei (Lesson, 1839) & Rufous-crested Coquette & $P$ & & CITES-II \\
\hline Lophornis stictolophus Salvin \& Elliot, 1873 & Spangled Coquette & PE & & CITES-II \\
\hline Lophornis verreauxii Bourcier, 1853 & Butterfly Coquette & $V * P R$ & FMNH, MUSM, USNM & CITES-II \\
\hline Phlogophilus hemileucurus Gould, 1860 & Ecuadorian Piedtail & $\mathrm{VP}$ & ROM & IUCN-VU, MIN-NT, CITES-II \\
\hline Adelomyia melanogenys (Fraser, 1840) & Speckled Hummingbird & VPR & MUSM & CITES-II \\
\hline Aglaiocercus kingii (Lesson, 1832) & Long-tailed Sylph & $P R$ & & CITES-II \\
\hline Eriocnemis aline (Bourcier, 1843) & Emerald-bellied Puffleg & VPE & $\mathrm{MCZ}$ & CITES-II \\
\hline Aglaeactis cupripennis (Bourcier, 1843) & Shining Sunbeam & $\mathrm{PE}$ & & CITES-II \\
\hline Coeligena coeligena (Lesson, 1833) & Bronzy Inca & $\mathrm{VP}$ & LSUMZ & CITES-II \\
\hline Ocreatus underwoodii (Lesson, 1832) & Booted Racket-tail & VP & LSUMZ & CITES-II \\
\hline Urochroa leucura Lawrence, 1864 & Green-backed Hillstar & $\mathrm{E}$ & & CITES-II \\
\hline Heliodoxa gularis (Gould, 1860) & Pink-throated Brilliant & $V * P$ & LSUMZ & IUCN-VU, CITES-II \\
\hline Heliodoxa branickii (Taczanowski, 1874) & Rufous-webbed Brilliant & $\mathrm{VP}$ & LSUMZ & CITES-II, end \\
\hline Heliodoxa schreibersii (Bourcier, 1847) & Black-throated Brilliant & $\mathrm{V} * \mathrm{PR}$ & LSUMZ, USNM & CITES-II \\
\hline Heliodoxa aurescens (Gould, 1846) & Gould's Jewelfront & $V * P R$ & FMNH, KU, LSUMZ, MUSM, TCWC, UMMZ & CITES-II \\
\hline
\end{tabular}


SALINAS ET AL.

\begin{tabular}{|c|c|c|c|c|}
\hline Nombre científico & Nombre en inglés & Validación & Procedencia de Voucher & Amenaza/endemismo \\
\hline Heliodoxa leadbeateri (Bourcier, 1843) & Violet-fronted Brilliant & $P$ & & CITES-II \\
\hline Heliomaster longirostris (Audebert \& Vieillot, 1801) & Long-billed Starthroat & $V * P R$ & FMNH, MNHN & CITES-II \\
\hline Chaetocercus mulsant (Bourcier, 1843) & White-bellied Woodstar & $\mathrm{P}$ & & CITES-II \\
\hline Calliphlox amethystina (Boddaert, 1783) & Amethyst Woodstar & $P$ & & CITES-II \\
\hline Chlorostilbon mellisugus (Linnaeus, 1758) & Blue-tailed Emerald & VPR & CLO, FMNH, LSUMZ, USNM, WFVZ & CITES-II \\
\hline Klais guimeti (Bourcier, 1843) & Violet-headed Hummingbird & $\mathrm{VP}$ & LSUMZ, MUSM & CITES-II \\
\hline Campylopterus largipennis (Boddaert, 1783) & Gray-breasted Sabrewing & V*PR & LSUMZ, FMNH, MUSM, UMMZ, USNM & CITES-II \\
\hline Campylopterus villaviscensio (Bourcier, 1851) & Napo Sabrewing & $\mathrm{VP}$ & MCZ, UWBM & IUCN-NT, MIN-NT, CITES-II \\
\hline Thalurania furcata (Gmelin, JF, 1788) & Fork-tailed Woodnymph & V*PR & $\begin{array}{l}\text { CLO, FMNH, KU, LSUMZ, MIZ, MUSM, MZBW, UF-FLMNH, UMMZ, Uni- } \\
\text { camp-FNJV, UWBM }\end{array}$ & CITES-II \\
\hline Taphrospilus hypostictus (Gould, 1862) & Many-spotted Hummingbird & $P$ & & CITES-II \\
\hline Talaphorus chlorocercus Gould, 1866 & Olive-spotted Hummingbird & V*PR & CLO, CM, FMNH, LSUMZ, MCZ, UF-FLMNH, UMMZ, USNM & CITES-II \\
\hline Uranomitra franciae (Bourcier \& Mulsant, 1846) & Andean Emerald & VR & LSUMZ & CITES-II \\
\hline Chrysuronia versicolor (Vieillot, 1818) & Versicolored Emerald & $\mathrm{PE}$ & & CITES-II \\
\hline Chrysuronia oenone (Lesson, 1832) & Golden-tailed Sapphire & $V^{*} P R$ & BMNH, LSUMZ, FMNH, MCZ, MUSM, UMMZ, USNM & CITES-II \\
\hline Chionomesa fimbriata (Gmelin, JF, 1788) & Glittering-throated Emerald & $V * P R$ & BMNH, CLO, CM, FMNH, LSUMZ, MCZ, MLZ, MUSM, UMMZ, USNM, YPM & CITES-II \\
\hline Chionomesa lactea (Lesson, 1832) & Sapphire-spangled Emerald & VPR & CLO, FMNH, LSUMZ, MUSM & CITES-II \\
\hline Hylocharis sapphirina (Gmelin, JF, 1788) & Rufous-throated Sapphire & VPR & LSUMZ & CITES-II \\
\hline Chlorestes cyanus (Vieillot, 1818) & White-chinned Sapphire & $V * P R$ & FMNH, LSUMZ, MUSM, UMMZ & CITES-II \\
\hline Chlorestes notata (Reich, 1793) & Blue-chinned Sapphire & $V * P R$ & LSUMZ, FMNH, MCZ, MLZ, MUSM, UMMZ & CITES-II \\
\hline \multicolumn{5}{|l|}{ Orden Opisthocomiformes: Familia Opisthocomidae } \\
\hline Opisthocomus hoazin (Statius Muller, 1776) & Hoatzin & V*PR & $\mathrm{AMNH}, \mathrm{CLO}, \mathrm{FMNH}, \mathrm{KU}, \mathrm{MCZ}$ & \\
\hline \multicolumn{5}{|l|}{ Orden Gruiformes: Familia Aramidae } \\
\hline Aramus guarauna (Linnaeus, 1766) & Limpkin & $\mathrm{P}$ & & \\
\hline \multicolumn{5}{|l|}{ Orden Gruiformes: Familia Psophiidae } \\
\hline Psophia crepitans Linnaeus, 1758 & Gray-winged Trumpeter & $\mathrm{V} * \mathrm{PR}$ & CLO, KU, LSUMZ, MCZ, UF-FLMNH, UMMZ & IUCN-NT \\
\hline Psophia leucoptera von Spix, 1825 & Pale-winged Trumpeter & V*PR & CLO, FMNH, LSUMZ, MUSM & IUCN-NT \\
\hline \multicolumn{5}{|l|}{ Orden Gruiformes: Familia Rallidae } \\
\hline Porphyrio martinica (Linnaeus, 1766) & Purple Gallinule & VPR & CM, LSUMZ, MUSM & \\
\hline Porphyrio flavirostris (Gmelin, JF, 1789) & Azure Gallinule & $\mathrm{P}$ & & \\
\hline Anurolimnas castaneiceps (Sclater \& Salvin, 1869) & Chestnut-headed Crake & VPR & CLO, LSUMZ, MUSM, UF-FLMNH & \\
\hline Anurolimnas viridis (Statius Muller, 1776) & Russet-crowned Crake & VPR & LSUMZ, MUSM & \\
\hline Anurolimnas fasciatus Sclater \& Salvin, 1868 & Black-banded Crake & $\mathrm{VP}$ & CLO, FMNH, MUSM & \\
\hline Laterallus melanophaius (Vieillot, 1819) & Rufous-sided Crake & VPR & MUSM & \\
\hline Laterallus exilis (Temminck, 1831) & Gray-breasted Crake & VPR & CM, LSUMZ, MUSM, UF-FLMNH & \\
\hline Mustelirallus albicollis (Vieillot, 1819) & Ash-throated Crake & VPR & LSUMZ & \\
\hline Mustelirallus erythrops (Sclater, PL, 1867) & Paint-billed Crake & VPR & MUSM & \\
\hline Pardirallus nigricans (Vieillot, 1819) & Blackish Rail & $\mathrm{PE}$ & & \\
\hline Amaurolimnas concolor (Gosse, 1847) & Uniform Crake & $\mathrm{VP}$ & FMNH & \\
\hline Aramides cajaneus (Statius Muller, 1776) & Gray-cowled Wood-Rail & $V * P R$ & AMNH, CLO, FMNH, KU, LSUMZ, MUSM, RMNH & \\
\hline Aramides calopterus Sclater \& Salvin, 1878 & Red-winged Wood-Rail & $\mathrm{VP}$ & MUSM & \\
\hline
\end{tabular}


LAS AVES DEL DEPARTAMENTO DE LORETO

\begin{tabular}{|c|c|c|c|c|}
\hline Nombre científico & Nombre en inglés & Validación & Procedencia de Voucher & Amenaza/endemismo \\
\hline Porzana carolina (Linnaeus, 1758) & Sora & $\mathrm{VP}$ & CLO & \\
\hline Gallinula galeata (Lichtenstein, MHC, 1818) & Common Gallinule & $\mathrm{P}$ & & \\
\hline \multicolumn{5}{|l|}{ Orden Gruiformes: Familia Heliornithidae } \\
\hline Heliornis fulica (Boddaert, 1783) & Sungrebe & $V * P R$ & CLO, FMNH, LSUMZ, MCZ, MUSM, YPM & \\
\hline \multicolumn{5}{|l|}{ Orden Charadriiformes: Familia Charadriidae } \\
\hline Pluvialis dominica (Statius Muller, 1776) & American Golden-Plover & VP & CLO & \\
\hline Vanellus cayanus (Latham, 1790) & Pied Lapwing & $V * P R$ & LSUMZ, MUSM & \\
\hline Vanellus chilensis (Molina, 1782) & Southern Lapwing & PR & & \\
\hline Charadrius vociferus Linnaeus, 1758 & Killdeer & $P R$ & & \\
\hline Charadrius collaris Vieillot, 1818 & Collared Plover & VPR & FMNH, LSUMZ, MUSM, USNM & \\
\hline \multicolumn{5}{|l|}{ Orden Charadriiformes: Familia Recurvirostridae } \\
\hline Himantopus mexicanus (Statius Muller, 1776) & Black-necked Stilt & $\mathrm{V*P}$ & MUSM & \\
\hline \multicolumn{5}{|l|}{ Orden Charadriiformes: Familia Scolopacidae } \\
\hline Bartramia longicauda (Bechstein, 1812) & Upland Sandpiper & $\mathrm{P}$ & & \\
\hline Arenaria interpres (Linnaeus, 1758) & Ruddy Turnstone & $P$ & & CMS-II \\
\hline Calidris himantopus (Bonaparte, 1826) & Stilt Sandpiper & VPR & CLO & \\
\hline Calidris alba (Pallas, 1764) & Sanderling & $P$ & & CMS-II \\
\hline Calidris bairdii (Coues, 1861) & Baird's Sandpiper & $P$ & & \\
\hline Calidris minutilla (Vieillot, 1819) & Least Sandpiper & $P$ & & \\
\hline Calidris fuscicollis (Vieillot, 1819) & White-rumped Sandpiper & $\mathrm{P}$ & & \\
\hline Calidris subruficollis (Vieillot, 1819) & Buff-breasted Sandpiper & VPR & LSUMZ, MUSM & IUCN-NT, CMS-I \\
\hline Calidris melanotos (Vieillot, 1819) & Pectoral Sandpiper & VPR & FMNH, KU, LSUMZ, MUSM & \\
\hline Calidris mauri (Cabanis, 1857) & Western Sandpiper & $P R$ & & \\
\hline Gallinago paraguaiae (Vieillot, 1816) & South American Snipe & $P R$ & & \\
\hline Phalaropus tricolor Vieillot, 1819 & Wilson's Phalarope & $P$ & & \\
\hline Actitis macularius (Linnaeus, 1766) & Spotted Sandpiper & $V * P R$ & LSUMZ & \\
\hline Tringa solitaria Wilson, A, 1813 & Solitary Sandpiper & V*PR & FMNH, LSUMZ, MUSM & \\
\hline Tringa melanoleuca (Gmelin, JF, 1789) & Greater Yellowlegs & PR & & \\
\hline Tring a flavipes (Gmelin, JF, 1789) & Lesser Yellowlegs & $V * P R$ & FMNH, MUSM & \\
\hline \multicolumn{5}{|l|}{ Orden Charadriiformes: Familia Jacanidae } \\
\hline Jacana jacana (Linnaeus, 1766) & Wattled Jacana & $V * P R$ & CLO, FMNH, LSUMZ, MCZ, MUSM, UF-FLMNH, YPM & \\
\hline \multicolumn{5}{|l|}{ Orden Charadriiformes: Familia Rynchopidae } \\
\hline Rynchops niger Linnaeus, 1758 & Black Skimmer & VPR & FMNH, LSUMZ, MUSM & \\
\hline \multicolumn{5}{|l|}{ Orden Charadriiformes: Familia Laridae } \\
\hline Leucophaeus atricilla (Linnaeus, 1758) & Laughing Gull & VPR & MUSM & \\
\hline Leucophaeus pipixcan (Wagler, 1831) & Franklin's Gull & PR & & \\
\hline Sternula superciliaris (Vieillot, 1819) & Yellow-billed Tern & $\mathrm{V} * \mathrm{PR}$ & FMNH, LSUMZ, MCZ, MUSM, MVZ & \\
\hline Phaetusa simplex (Gmelin, JF, 1789) & Large-billed Tern & $V * P R$ & CLO, CM, FMNH, LSUMZ, MCZ, MUSM, UMMZ, USNM & \\
\hline Chlidonias niger (Linnaeus, 1758) & Black Tern & $P R$ & & CMS-II \\
\hline Sterna hirundo Linnaeus, 1758 & Common Tern & $P R$ & & CMS-II \\
\hline
\end{tabular}


SALINAS ET AL.

\begin{tabular}{|c|c|c|c|c|}
\hline Nombre científico & Nombre en inglés & Validación & Procedencia de Voucher & Amenaza/endemismo \\
\hline \multicolumn{5}{|l|}{ Orden Eurypygiformes: Familia Eurypygidae } \\
\hline Eurypyga helias (Pallas, 1781) & Sunbittern & $V * P R$ & CLO, FMNH, LSUMZ, MUSM & \\
\hline \multicolumn{5}{|l|}{ Orden Ciconiiformes: Familia Ciconiidae } \\
\hline Ciconia maguari (Gmelin, JF, 1789) & Maguari Stork & $P R$ & & \\
\hline Jabiru mycteria (Lichtenstein, MHC, 1819) & Jabiru & VPR & LSUMZ & MIN-NT, CITES-I \\
\hline Mycteria americana Linnaeus, 1758 & Wood Stork & $\mathrm{V*PR}$ & MUSM & MIN-NT \\
\hline \multicolumn{5}{|l|}{ Orden Suliformes: Familia Anhingidae } \\
\hline Anhinga anhinga (Linnaeus, 1766) & Anhinga & V*PR & FMNH, MCZ, MUSM & \\
\hline \multicolumn{5}{|l|}{ Orden Suliformes: Familia Phalacrocoracidae } \\
\hline Phalacrocorax brasilianus (Gmelin, JF, 1789) & Neotropic Cormorant & VPR & LSUMZ & \\
\hline \multicolumn{5}{|l|}{ Orden Pelecaniformes: Familia Ardeidae } \\
\hline Tigrisoma lineatum (Boddaert, 1783) & Rufescent Tiger-Heron & $\mathrm{V} * \mathrm{PR}$ & CLO, FMNH, LSUMZ, MCZ, MUSM & \\
\hline Tigrisoma fasciatum (Such, 1825) & Fasciated Tiger-Heron & VP & LSUMZ & \\
\hline Agamia agami (Gmelin, JF, 1789) & Agami Heron & V*PR & FMNH, LSUMZ, MUSM & IUCN-VU \\
\hline Cochlearius cochlearius (Linnaeus, 1766) & Boat-billed Heron & V*PR & FMNH, LSUMZ, MUSM & \\
\hline Zebrilus undulatus (Gmelin, JF, 1789) & Zigzag Heron & VPR & MUSM & IUCN-NT \\
\hline Botaurus pinnatus (Wagler, 1829) & Pinnated Bittern & $P R$ & & \\
\hline Ixobrychus exilis (Gmelin, JF, 1789) & Least Bittern & $\mathrm{VP}$ & $\mathrm{CLO}$ & \\
\hline Nycticorax nycticorax (Linnaeus, 1758) & Black-crowned Night-Heron & $P R$ & & \\
\hline Butorides striata (Linnaeus, 1758) & Striated Heron & VPR & CM, FMNH, LSUMZ, MUSM, MVZ, YPM & \\
\hline Bubulcus ibis (Linnaeus, 1758) & Cattle Egret & VPR & FMNH, MUSM & \\
\hline Ardea cocoi Linnaeus, 1766 & Cocoi Heron & $\mathrm{V} * \mathrm{PR}$ & MUSM & \\
\hline Ardea alba Linnaeus, 1758 & Great Egret & V*PR & FMNH, MUSM & CMS-II \\
\hline Syrigma sibilatrix (Temminck, 1824) & Whistling Heron & $P$ & & \\
\hline Pilherodius pileatus (Boddaert, 1783) & Capped Heron & VPR & LSUMZ, YPM & \\
\hline Egretta thula (Molina, 1782) & Snowy Egret & $\mathrm{V*PR}$ & RMNH & \\
\hline Egretta caerulea (Linnaeus, 1758) & Little Blue Heron & VPR & FMNH & \\
\hline \multicolumn{5}{|l|}{ Orden Pelecaniformes: Familia Threskiornithidae } \\
\hline Eudocimus albus (Linnaeus, 1758) & White lbis & $P$ & & \\
\hline Eudocimus ruber (Linnaeus, 1758) & Scarlet Ibis & $P R$ & & CITES-II \\
\hline Mesembrinibis cayennensis (Gmelin, JF, 1789) & Green Ibis & V*PR & MUSM & \\
\hline Phimosus infuscatus (Lichtenstein, MHC, 1823) & Bare-faced Ibis & $P$ & & \\
\hline Platalea ajaja Linnaeus, 1758 & Roseate Spoonbill & $\mathrm{PR}$ & & MIN-NT \\
\hline \multicolumn{5}{|l|}{ Orden Cathartiformes: Familia Cathartidae } \\
\hline Sarcoramphus papa (Linnaeus, 1758) & King Vulture & V*PR & FMNH & CITES-III, CMS-II \\
\hline Coragyps atratus (Bechstein, 1793) & Black Vulture & VPR & CM, MUSM & CMS-II \\
\hline Cathartes aura (Linnaeus, 1758) & Turkey Vulture & VPR & CM, LSUMZ, MUSM & CMS-II \\
\hline Cathartes burrovianus Cassin, 1845 & Lesser Yellow-headed Vulture & V*PR & MUSM & CMS-II \\
\hline Cathartes melambrotus Wetmore, 1964 & Greater Yellow-headed Vulture & VPR & LSUMZ & CMS-II \\
\hline Orden Accipitriformes: Familia Pandionidae & & & & \\
\hline
\end{tabular}


LAS AVES DEL DEPARTAMENTO DE LORETO

\begin{tabular}{|c|c|c|c|c|}
\hline Nombre científico & Nombre en inglés & Validación & Procedencia de Voucher & Amenaza/endemismo \\
\hline Pandion haliaetus (Linnaeus, 1758) & Osprey & $V^{*} \mathrm{PR}$ & MNHN & CITES-II, CMS-II \\
\hline \multicolumn{5}{|l|}{ Orden Accipitriformes: Familia Accipitridae } \\
\hline Gampsonyx swainsonii Vigors, 1825 & Pearl Kite & VPR & CLO & CITES-II \\
\hline Chondrohierax uncinatus (Temminck, 1822) & Hook-billed Kite & $\mathrm{V} * \mathrm{PR}$ & FMNH & CITES-II \\
\hline Leptodon cayanensis (Latham, 1790) & Gray-headed Kite & $V * P R$ & LSUMZ, MCZ, MUSM & CITES-II \\
\hline Elanoides forficatus (Linnaeus, 1758) & Swallow-tailed Kite & $V * P R$ & FMNH, LSUMZ, MUSM & CITES-II \\
\hline Morphnus guianensis (Daudin, 1800) & Crested Eagle & $V * P R$ & CLO, LSUMZ & IUCN-NT, MIN-VU, CITES-II \\
\hline Harpia harpyja (Linnaeus, 1758) & Harpy Eagle & $V * P R$ & LSUMZ & IUCN-NT, MIN-VU, CITES-I \\
\hline Spizaetus tyrannus (zu Wied-Neuwied, 1820) & Black Hawk-Eagle & $\mathrm{V} * \mathrm{PR}$ & CLO, FMNH & CITES-II \\
\hline Spizaetus melanoleucus (Vieillot, 1816) & Black-and-white Hawk-Eagle & PR & & CITES-II \\
\hline Spizaetus ornatus (Daudin, 1800) & Ornate Hawk-Eagle & VPR & CLO & IUCN-NT, CITES-II \\
\hline Busarellus nigricollis (Latham, 1790) & Black-collared Hawk & $\mathrm{V} * \mathrm{PR}$ & FMNH, LSUMZ, MUSM, MVZ & CITES-II \\
\hline Rostrhamus sociabilis (Vieillot, 1817) & Snail Kite & VPR & LSUMZ, MUSM & CITES-II \\
\hline Helicolestes hamatus (Temminck, 1821) & Slender-billed Kite & VP & $\mathrm{CLO}, \mathrm{FMNH}$ & CITES-II \\
\hline Harpagus bidentatus (Latham, 1790) & Double-toothed Kite & $V * P R$ & CLO, FMNH, LSUMZ, MUSM & CITES-II \\
\hline Ictinia mississippiensis (Wilson, A, 1811) & Mississippi Kite & $P$ & & CITES-II \\
\hline Ictinia plumbea (Gmelin, JF, 1788) & Plumbeous Kite & $V * P R$ & CM, LSUMZ & CITES-II \\
\hline Accipiter poliogaster (Temminck, 1824) & Gray-bellied Hawk & $\mathrm{VP}$ & LSUMZ & IUCN-NT, CITES-II \\
\hline Accipiter superciliosus (Linnaeus, 1766) & Tiny Hawk & VPR & FMNH, LSUMZ, MUSM & CITES-II \\
\hline Accipiter striatus Vieillot, 1808 & Sharp-shinned Hawk & $\mathrm{VP}$ & LSUMZ, MUSM & CITES-II \\
\hline Accipiter bicolor (Vieillot, 1817) & Bicolored Hawk & V*PR & LSUMZ & CITES-II \\
\hline Geranospiza caerulescens (Vieillot, 1817) & Crane Hawk & VPR & CLO, FMNH, LSUMZ, MUSM, MVZ & CITES-II \\
\hline Buteogallus schistaceus (Sundevall, 1850) & Slate-colored Hawk & $\mathrm{V} * \mathrm{PR}$ & CLO, FMNH, LSUMZ & CITES-II \\
\hline Buteogallus urubitinga (Gmelin, JF, 1788) & Great Black Hawk & V*PR & LSUMZ, FMNH, MUSM & CITES-II \\
\hline Morphnarchus princeps (Sclater, PL, 1865) & Barred Hawk & $P R$ & & CITES-II \\
\hline Rupornis magnirostris (Gmelin, JF, 1788) & Roadside Hawk & $V * P R$ & CLO, CM, FMNH, LSUMZ, MUSM & CITES-II \\
\hline Pseudastur albicollis (Latham, 1790) & White Hawk & VPR & LSUMZ & CITES-II \\
\hline Leucopternis semiplumbeus Lawrence, 1861 & Semiplumbeous Hawk & $P R$ & & CITES-II \\
\hline Leucopternis melanops (Latham, 1790) & Black-faced Hawk & VPR & CLO, LSUMZ, MUSM & CITES-II \\
\hline Leucopternis kuhli Bonaparte, 1850 & White-browed Hawk & $V * P R$ & FMNH, LSUMZ & CITES-II \\
\hline Buteo nitidus (Latham, 1790) & Gray-lined Hawk & VP & LSUMZ & CITES-II \\
\hline Buteo platypterus (Vieillot, 1823) & Broad-winged Hawk & $\mathrm{V*P}$ & CM, FMNH & CITES-II \\
\hline Buteo albigula Philippi Sr, RA, 1899 & White-throated Hawk & $R$ & & CITES-II \\
\hline Buteo brachyurus Vieillot, 1816 & Short-tailed Hawk & $P R$ & & CITES-II \\
\hline Buteo swainsoni Bonaparte, 1838 & Swainson's Hawk & $P R$ & & CITES-II \\
\hline Buteo albonotatus Kaup, 1847 & Zone-tailed Hawk & PR & & CITES-II \\
\hline \multicolumn{5}{|l|}{ Orden Strigiformes: Familia Tytonidae } \\
\hline Tyto alba (Scopoli, 1769) & Barn Owl & $P$ & & CITES-II \\
\hline \multicolumn{5}{|l|}{ Orden Strigiformes: Familia Strigidae } \\
\hline Megascops choliba (Vieillot, 1817) & Tropical Screech-Owl & $V * P R$ & CLO, CM, FMNH, LSUMZ, MUSM, UF-FLMNH & CITES-II \\
\hline
\end{tabular}


SALINAS ET AL.

\begin{tabular}{|c|c|c|c|c|}
\hline Nombre científico & Nombre en inglés & Validación & Procedencia de Voucher & Amenaza/endemismo \\
\hline Megascops ingens (Salvin, 1897) & Rufescent Screech-Owl & $\mathrm{VP}$ & LSUMZ, MUSM & CITES-II \\
\hline Megascops roraimae (Salvin, 1897) & Foothill Screech-Owl & $R$ & & CITES-II \\
\hline Megascops watsonii (Cassin, 1849) & Tawny-bellied Screech-Owl & V*PR & CLO, FMNH, KU, LSUMZ, MUSM, UMMZ, YPM & CITES-II \\
\hline Lophostrix cristata (Daudin, 1800) & Crested Owl & $V * P R$ & CLO, LSUMZ, MUSM & CITES-II \\
\hline Pulsatrix perspicillata (Latham, 1790) & Spectacled Owl & VPR & CLO, LSUMZ, MUSM & CITES-II \\
\hline Pulsatrix melanota (von Tschudi, 1844) & Band-bellied Owl & $\mathrm{P}$ & & CITES-II \\
\hline Bubo virginianus (Gmelin, JF, 1788) & Great Horned Owl & $P R$ & & CITES-II \\
\hline Ciccaba virgata (Cassin, 1849) & Mottled Owl & V*PR & CLO, LSUMZ, MUSM & CITES-II \\
\hline Ciccaba huhula (Daudin, 1800) & Black-banded Owl & VPR & CLO, MUSM, UF-FLMNH & CITES-II \\
\hline Glaucidium parkeri Robbins \& Howell, 1995 & Subtropical Pygmy-Owl & VP & CLO, LSUMZ & CITES-II \\
\hline Glaucidium hardyi Vielliard, 1989 & Amazonian Pygmy-Owl & VPR & CLO, LSUMZ & CITES-II \\
\hline Glaucidium brasilianum (Gmelin, JF, 1788) & Ferruginous Pygmy-Owl & VPR & CLO, FMNH, LSUMZ, MUSM & CITES-II \\
\hline Athene cunicularia (Molina, 1782) & Burrowing Owl & $P$ & & CITES-II \\
\hline Aegolius harrisii (Cassin, 1849) & Buff-fronted Owl & $\mathrm{VP}$ & LSUMZ & CITES-II \\
\hline Asio clamator (Vieillot, 1808) & Striped Owl & VPR & LSUMZ, MUSM & CITES-II \\
\hline \multicolumn{5}{|l|}{ Orden Trogoniformes: Familia Trogonidae } \\
\hline Pharomachrus pavoninus (von Spix, 1824) & Pavonine Quetzal & $\mathrm{V} * \mathrm{PR}$ & CLO, FMNH, LSUMZ, MUSM, MVZ, UF-FLMNH & \\
\hline Pharomachrus auriceps (Gould, 1842) & Golden-headed Quetzal & $\mathrm{PE}$ & & \\
\hline Pharomachrus antisianus (d'Orbigny, 1837) & Crested Quetzal & $\mathrm{VP}$ & LSUMZ, MUSM & \\
\hline Trogon melanurus Swainson, 1838 & Black-tailed Trogon & V*PR & CLO, CM, FMNH, KU, LSUMZ, MUSM, UF-FLMNH & \\
\hline Trogon viridis Linnaeus, 1766 & Green-backed Trogon & V*PR & CLO, FMNH, KU, LSUMZ, MNHN, MUSM, MVZ & \\
\hline Trogon ramonianus Deville \& Des Murs, 1849 & Amazonian Trogon & V*PR & CLO, CM, KU, LSUMZ, MUSM, UF-FLMNH & \\
\hline Trogon curucui Linnaeus, 1766 & Blue-crowned Trogon & VPR & CLO, LSUMZ, MUSM & \\
\hline Trogon rufus Gmelin, JF, 1788 & Black-throated Trogon & V*PR & CLO, FMNH, LSUMZ, MUSM & \\
\hline Trogon collaris Vieillot, 1817 & Collared Trogon & $V * P R$ & CLO, CM, FMNH, LSUMZ, MUSM, UF-FLMNH, YPM & \\
\hline Trogon personatus Gould, 1842 & Masked Trogon & $R$ & & \\
\hline \multicolumn{5}{|l|}{ Orden Coraciiformes: Familia Momotidae } \\
\hline Electron platyrhynchum (Leadbeater, 1829) & Broad-billed Motmot & VPR & CLO, FMNH, KU, LSUMZ, MUSM, YPM & \\
\hline Baryphthengus martii (von Spix, 1824) & Rufous Motmot & $\mathrm{V} * \mathrm{PR}$ & CLO, FMNH, KU, LSUMZ, MUSM, UF-FLMNH, YPM & \\
\hline Momotus momota (Linnaeus, 1766) & Amazonian Motmot & $\mathrm{V} * \mathrm{PR}$ & CLO, FMNH, KU, LSUMZ, MUSM, UF-FLMNH & \\
\hline \multicolumn{5}{|l|}{ Orden Coraciiformes: Familia Alcedinidae } \\
\hline Megaceryle torquata (Linnaeus, 1766) & Ringed Kingfisher & $\mathrm{V*PR}$ & CLO, CM, CUMV, FMNH, LSUMZ, MUSM, MVZ, UMMZ & \\
\hline Chloroceryle amazona (Latham, 1790) & Amazon Kingfisher & V*PR & CLO, FMNH, LSUMZ, MUSM, MVZ, USNM & \\
\hline Chloroceryle aenea (Pallas, 1764) & American Pygmy Kingfisher & V*PR & CLO, CM, FMNH, LSUMZ, MUSM & \\
\hline Chloroceryle americana (Gmelin, JF, 1788) & Green Kingfisher & $\mathrm{V}$ PR & CLO, FMNH, KU, LSUMZ, MCZ, MUSM, UF-FLMNH, UMMZ & \\
\hline Chloroceryle inda (Linnaeus, 1766) & Green-and-rufous Kingfisher & V*PR & CLO, CM, FMNH, LSUMZ, MCZ, MUSM, UF-FLMNH, UMMZ & \\
\hline \multicolumn{5}{|l|}{ Orden Galbuliformes: Familia Galbulidae } \\
\hline Galbalcyrhynchus leucotis Des Murs, 1845 & White-eared Jacamar & $\mathrm{V*PR}$ & CLO, CM, FMNH, LSUMZ, MUSM, ROM, UF-FLMNH, YPM & \\
\hline Galbalcyrhynchus purusianus Goeldi, 1904 & Purus Jacamar & $\mathrm{VP}$ & LSUMZ, MUSM & \\
\hline Brachygalba albogularis (von Spix, 1824) & White-throated Jacamar & VPR & LSUMZ, MUSM & \\
\hline
\end{tabular}


LAS AVES DEL DEPARTAMENTO DE LORETO

\begin{tabular}{|c|c|c|c|c|}
\hline Nombre científico & Nombre en inglés & Validación & Procedencia de Voucher & Amenaza/endemismo \\
\hline Brachygalba lugubris (Swainson, 1838) & Brown Jacamar & $\mathrm{V} * \mathrm{PR}$ & MUSM & \\
\hline Galbula albirostris Latham, 1790 & Yellow-billed Jacamar & $\mathrm{V} * \mathrm{PR}$ & CLO, FMNH, KU, LSUMZ, MUSM, UMMZ & \\
\hline Galbula cyanicollis Cassin, 1851 & Blue-cheeked Jacamar & VPR & FMNH, LSUMZ, MUSM & \\
\hline Galbula tombacea von Spix, 1824 & White-chinned Jacamar & $\mathrm{V} * \mathrm{PR}$ & CLO, LSUMZ, MUSM, UMMZ & \\
\hline Galbula cyanescens Deville, 1849 & Bluish-fronted Jacamar & VPR & FMNH, LSUMZ, MUSM & \\
\hline Galbula chalcothorax Sclater, PL, 1855 & Purplish Jacamar & $\mathrm{V*PR}$ & LSUMZ & \\
\hline Galbula dea (Linnaeus, 1758) & Paradise Jacamar & $\mathrm{V} * \mathrm{PR}$ & LSUMZ, MUSM & \\
\hline Jacamerops aureus (Statius Muller, 1776) & Great Jacamar & $\mathrm{V} * \mathrm{PR}$ & CLO, FMNH, KU, LSUMZ, MCZ, MUSM & \\
\hline \multicolumn{5}{|l|}{ Orden Galbuliformes: Familia Bucconidae } \\
\hline Notharchus hyperrhynchus (Sclater, PL, 1856) & White-necked Puffbird & $\mathrm{V*PR}$ & CLO, LSUMZ, MUSM & \\
\hline Notharchus ordii (Cassin, 1851) & Brown-banded Puffbird & $P R$ & & \\
\hline Notharchus tectus (Boddaert, 1783) & Pied Puffbird & $\mathrm{V*PR}$ & CLO, LSUMZ, MUSM & \\
\hline Bucco macrodactylus (von Spix, 1824) & Chestnut-capped Puffbird & $\mathrm{V} * \mathrm{PR}$ & CLO, FMNH, LSUMZ, MUSM, UMMZ & \\
\hline Bucco tamatia Gmelin, JF, 1788 & Spotted Puffbird & $\mathrm{V} * \mathrm{PR}$ & CLO, LSUMZ, MUSM, UF-FLMNH & \\
\hline Bucco capensis Linnaeus, 1766 & Collared Puffbird & VPR & CLO, FMNH, KU, LSUMZ, MCZ, MUSM & \\
\hline Nystalus obamai Whitney et al., 2013 & Western Striolated-Puffbird & VPR & CLO, LSUMZ, MUSM & \\
\hline Malacoptila fusca (Gmelin, JF, 1788) & White-chested Puffbird & $\mathrm{V} * \mathrm{PR}$ & CLO, KU, LSUMZ, MUSM & \\
\hline Malacoptila semicincta Todd, 1925 & Semicollared Puffbird & VPR & MUSM & \\
\hline Malacoptila rufa (von Spix, 1824) & Rufous-necked Puffbird & $\mathrm{V} * \mathrm{PR}$ & FMNH, LSUMZ, MCZ, MUSM & \\
\hline Malacoptila fulvogularis Sclater, PL, 1854 & Black-streaked Puffbird & $P R$ & & \\
\hline Micromonacha lanceolata (Deville, 1849) & Lanceolated Monklet & VPR & LSUMZ, MCZ & \\
\hline Nonnula rubecula (von Spix, 1824) & Rusty-breasted Nunlet & VP & CLO, LSUMZ, MUSM & \\
\hline Nonnula sclateri Hellmayr, 1907 & Fulvous-chinned Nunlet & $\mathrm{VP}$ & LSUMZ, MUSM & \\
\hline Nonnula brunnea Sclater, PL, 1881 & Brown Nunlet & $\mathrm{V} * \mathrm{PR}$ & KU, LSUMZ, MUSM & \\
\hline Nonnula ruficapilla (von Tschudi, 1844) & Rufous-capped Nunlet & $\mathrm{V} * \mathrm{PR}$ & FMNH, LSUMZ, MUSM & \\
\hline Monasa nigrifrons (von Spix, 1824) & Black-fronted Nunbird & $\mathrm{V} * \mathrm{PR}$ & CLO, CM, FMNH, LSUMZ, MUSM, UF-FLMNH, YPM & \\
\hline Monasa morphoeus (Hahn \& Küster, 1823) & White-fronted Nunbird & $\mathrm{V} * \mathrm{PR}$ & CLO, FMNH, LSUMZ, MUSM & \\
\hline Monasa flavirostris Strickland, 1850 & Yellow-billed Nunbird & $\mathrm{V} * \mathrm{PR}$ & CLO, CM, KU, LSUMZ, MUSM & \\
\hline Chelidoptera tenebrosa (Pallas, 1782) & Swallow-winged Puffbird & $\mathrm{V} * \mathrm{PR}$ & CM, FMNH, KU, LSUMZ, MCZ, MUSM, YPM & \\
\hline \multicolumn{5}{|l|}{ Orden Piciformes: Familia Capitonidae } \\
\hline Capito aurovirens (Cuvier, 1829) & Scarlet-crowned Barbet & $\mathrm{V} * \mathrm{PR}$ & CLO, CM, FMNH, LSUMZ, MUSM, UF-FLMNH & \\
\hline Capito wallacei O'Neill, Lane, Kratter, Capparella \& Fox, 2000 & Scarlet-banded Barbet & $\mathrm{VP}$ & CLO, LSUMZ, MUSM, USNM & IUCN-VU, MIN-VU, end \\
\hline Capito auratus (Dumont, 1816) & Gilded Barbet & $\mathrm{V*PR}$ & CLO, CM, FMNH, LSUMZ, MCZ, MUSM, ROM, UF-FLMNH, YPM & \\
\hline Eubucco richardsoni (Gray, GR, 1846) & Lemon-throated Barbet & $\mathrm{VP}$ & CM, FMNH, LSUMZ, MUSM, UF-FLMNH & \\
\hline Eubucco tucinkae (von Seilern, 1913) & Scarlet-hooded Barbet & $P E$ & & \\
\hline Eubucco versicolor (Statius Muller, 1776) & Versicolored Barbet & $\mathrm{V*p}$ & CLO, LSUMZ, MUSM & \\
\hline \multicolumn{5}{|l|}{ Orden Piciformes: Familia Ramphastidae } \\
\hline Ramphastos ambiguus Swainson, 1823 & Yellow-throated Toucan & $\mathrm{P}$ & & IUCN-NT, MIN-NT \\
\hline Ramphastos tucanus Linnaeus, 1758 & White-throated Toucan & $\mathrm{V} * \mathrm{PR}$ & CLO, FMNH, KU, LSUMZ, MUSM, MVZ, RMNH & IUCN-VU, CITES-II \\
\hline Ramphastos vitellinus Lichtenstein, MHC, 1823 & Channel-billed Toucan & $\mathrm{V} * \mathrm{PR}$ & CLO, FMNH, LSUMZ, MUSM, UF-FLMNH, YPM & IUCN-VU, CITES-II \\
\hline
\end{tabular}


SALINAS ET AL.

\begin{tabular}{|c|c|c|c|c|}
\hline Nombre científico & Nombre en inglés & Validación & Procedencia de Voucher & Amenaza/endemismo \\
\hline Aulacorhynchus prasinus (Gould, 1833) & Emerald Toucanet & $R$ & & \\
\hline Aulacorhynchus derbianus Gould, 1835 & Chestnut-tipped Toucanet & $\mathrm{VP}$ & CLO, LSUMZ, MUSM, UF-FLMNH & \\
\hline Selenidera reinwardtii (Wagler, 1827) & Golden-collared Toucanet & $\mathrm{V}$ PR & CLO, CM, FMNH, KU, LSUMZ, MNHN, MUSM, YPM & \\
\hline Pteroglossus inscriptus Swainson, 1822 & Lettered Aracari & $V * P R$ & CLO, FMNH, LSUMZ, MNHN, MUSM, MVZ, YPM & \\
\hline Pteroglossus castanotis Gould, 1834 & Chestnut-eared Aracari & $\mathrm{V}$ PR & CLO, CM, FMNH, LSUMZ, MCZ, MUSM, MVZ, RMNH, YPM & CITES-III \\
\hline Pteroglossus pluricinctus Gould, 1835 & Many-banded Aracari & $\mathrm{V} * \mathrm{PR}$ & CLO, LSUMZ, MNHN, MUSM, USNM & \\
\hline Pteroglossus azara (Vieillot, 1819) & Ivory-billed Aracari & V*PR & CLO, FMNH, LSUMZ, MNHN, MUSM, USNM & \\
\hline Pteroglossus beauharnaesii Wagler, 1832 & Curl-crested Aracari & $V * P R$ & FMNH, LSUMZ, MUSM, ROM & MIN-NT \\
\hline \multicolumn{5}{|l|}{ Orden Piciformes: Familia Picidae } \\
\hline Picumnus aurifrons von Pelzeln, 1870 & Bar-breasted Piculet & VPR & LSUMZ, MUSM & \\
\hline Picumnus lafresnayi Malherbe, 1862 & Lafresnaye's Piculet & VPR & LSUMZ & \\
\hline Picumnus rufiventris (Bonaparte, 1838) & Rufous-breasted Piculet & $V * P R$ & FMNH, LSUMZ, MCZ, MUSM & \\
\hline Picumnus castelnau Malherbe, 1862 & Plain-breasted Piculet & $V * P R$ & CLO, FMNH, LSUMZ, MUSM & \\
\hline Melanerpes cruentatus (Boddaert, 1783) & Yellow-tufted Woodpecker & $V * P R$ & CM, FMNH, LSUMZ, MCZ, MUSM, MVZ, UMMZ & \\
\hline Dryobates fumigatus (d'Orbigny, 1840) & Smoky-brown Woodpecker & $\mathrm{VP}$ & LSUMZ, MUSM & \\
\hline Dryobates passerinus (Linnaeus, 1766) & Little Woodpecker & VPR & FMNH, LSUMZ, MUSM & \\
\hline Dryobates dignus (Sclater \& Salvin, 1877) & Yellow-vented Woodpecker & $R$ & & \\
\hline Dryobates affinis (Swainson, 1821) & Red-stained Woodpecker & $\mathrm{V}$ PR & CLO, FMNH, LSUMZ, MUSM & \\
\hline Campephilus haematogaster (von Tschudi, 1844) & Crimson-bellied Woodpecker & VPE & LSUMZ & \\
\hline Campephilus rubricollis (Boddaert, 1783) & Red-necked Woodpecker & V*PR & CLO, FMNH, KU, LSUMZ, MUSM, UF-FLMNH & \\
\hline Campephilus melanoleucos (Gmelin, JF, 1788) & Crimson-crested Woodpecker & $\mathrm{V}$ PR & CLO, FMNH, LSUMZ, MUSM, YPM & \\
\hline Dryocopus lineatus (Linnaeus, 1766) & Lineated Woodpecker & VPR & CLO, FMNH, LSUMZ, MUSM & \\
\hline Celeus torquatus (Boddaert, 1783) & Ringed Woodpecker & VPR & CLO, LSUMZ, MUSM, UF-FLMNH & IUCN-NT \\
\hline Celeus grammicus (Natterer \& Malherbe, 1845) & Scale-breasted Woodpecker & $\mathrm{V} * \mathrm{PR}$ & CLO, CM, LSUMZ, MUSM & \\
\hline Celeus flavus (Statius Muller, 1776) & Cream-colored Woodpecker & $\mathrm{V} * \mathrm{PR}$ & CLO, FMNH, KU, LSUMZ, MCZ, MUSM, UMMZ, YPM & \\
\hline Celeus spectabilis Sclater \& Salvin, 1880 & Rufous-headed Woodpecker & VPR & FMNH, LSUMZ, MUSM & \\
\hline Celeus elegans (Statius Muller, 1776) & Chestnut Woodpecker & $\mathrm{V} * \mathrm{PR}$ & CLO, CM, FMNH, KU, LSUMZ, MUSM, YPM & \\
\hline Piculus leucolaemus (Natterer \& Malherbe, 1845) & White-throated Woodpecker & $\mathrm{VP}$ & LSUMZ, MUSM & \\
\hline Piculus flavigula (Boddaert, 1783) & Yellow-throated Woodpecker & V*PR & CLO, FMNH, LSUMZ, MNHN, MUSM & \\
\hline Piculus chrysochloros (Vieillot, 1818) & Golden-green Woodpecker & VPR & FMNH, LSUMZ, MUSM & \\
\hline Colaptes rubiginosus (Swainson, 1820) & Golden-olive Woodpecker & $\mathrm{VP}$ & LSUMZ, MUSM & \\
\hline Colaptes punctigula (Boddaert, 1783) & Spot-breasted Woodpecker & VPR & CLO, CM, FMNH, KU, LSUMZ, MCZ, MUSM & \\
\hline \multicolumn{5}{|l|}{ Orden Falconiformes: Familia Falconidae } \\
\hline Herpetotheres cachinnans (Linnaeus, 1758) & Laughing Falcon & VPR & CLO, FMNH, LSUMZ, MUSM, UF-FLMNH & CITES-II \\
\hline Micrastur ruficollis (Vieillot, 1817) & Barred Forest-Falcon & VPR & CLO, LSUMZ, MUSM & CITES-II \\
\hline Micrastur gilvicollis (Vieillot, 1817) & Lined Forest-Falcon & $\mathrm{V} * \mathrm{PR}$ & CLO, FMNH, LSUMZ, MUSM, YPM & CITES-II \\
\hline Micrastur mirandollei (Schlegel, 1862) & Slaty-backed Forest-Falcon & V*PR & CLO, LSUMZ, MUSM & CITES-II \\
\hline Micrastur semitorquatus (Vieillot, 1817) & Collared Forest-Falcon & $V * P R$ & MUSM & CITES-II \\
\hline Micrastur buckleyi Swann, 1919 & Buckley's Forest-Falcon & $P R$ & & CITES-II \\
\hline Caracara plancus (Miller, JF, 1777) & Crested Caracara & VPR & MUSM & CITES-II \\
\hline
\end{tabular}


LAS AVES DEL DEPARTAMENTO DE LORETO

\begin{tabular}{|c|c|c|c|c|}
\hline Nombre científico & Nombre en inglés & Validación & Procedencia de Voucher & Amenaza/endemismo \\
\hline lbycter americanus (Boddaert, 1783) & Red-throated Caracara & $V^{*} P R$ & CLO, FMNH, LSUMZ, MUSM & CITES-II \\
\hline Daptrius ater Vieillot, 1816 & Black Caracara & $V * P R$ & FMNH, LSUMZ, MUSM & CITES-II \\
\hline Milvago chimachima (Vieillot, 1816) & Yellow-headed Caracara & $V * P R$ & CLO, CM, FMNH, LSUMZ, MCZ, MUSM & CITES-II \\
\hline Falco columbarius Linnaeus, 1758 & Merlin & P & & CITES-II, CMS-II \\
\hline Falco rufigularis Daudin, 1800 & Bat Falcon & VPR & CLO, LSUMZ, MCZ, MUSM & CITES-II \\
\hline Falco deiroleucus Temminck, 1825 & Orange-breasted Falcon & PR & & IUCN-NT, MIN-NT, CITES-II \\
\hline Falco peregrinus Tunstall, 1771 & Peregrine Falcon & $P R$ & & MIN-NT, CITES-I, CMS-II \\
\hline \multicolumn{5}{|l|}{ Orden Psittaciformes: Familia Psittacidae } \\
\hline Touit huetii (Temminck, 1830) & Scarlet-shouldered Parrotlet & $\mathrm{V} * \mathrm{PR}$ & LSUMZ, MUSM & IUCN-VU, CITES-II \\
\hline Touit purpuratus (Gmelin, JF, 1788) & Sapphire-rumped Parrotlet & $\mathrm{VP}$ & $\mathrm{CLO}$ & CITES-II \\
\hline Touit stictopterus (Sclater, PL, 1862) & Spot-winged Parrotlet & $\mathrm{VP}$ & $\mathrm{CLO}$ & IUCN-VU, MIN-VU, CITES-II \\
\hline Nannopsittaca dachilleae O'Neill, Munn \& Franke, 1991 & Amazonian Parrotlet & $p$ & & IUCN-NT, MIN-NT, CITES-II \\
\hline Brotogeris sanctithomae (Statius Muller, 1776) & Tui Parakeet & $V^{*} P R$ & CLO, LSUMZ, MCZ, MNHN, MUSM & CITES-II \\
\hline Brotogeris versicolurus (Statius Muller, 1776) & Canary-winged Parakeet & $V * P R$ & CLO, FMNH, LSUMZ, MCZ, MUSM, UF-FLMNH, YPM & CITES-II \\
\hline Brotogeris cyanoptera (von Pelzeln, 1870) & Cobalt-winged Parakeet & $\mathrm{V*PR}$ & CLO, FMNH, LSUMZ, MCZ, MNHN, MUSM, YPM & CITES-II \\
\hline Pyrilia barrabandi (Kuhl, 1820) & Orange-cheeked Parrot & $\mathrm{V*PR}$ & $\mathrm{CM}, \mathrm{FMNH}, \mathrm{LACM}, \mathrm{LSUMZ}, \mathrm{MCZ}, \mathrm{MNHN}, \mathrm{MUSM}, \mathrm{UMMZ}$, YPM & IUCN-NT, CITES-II \\
\hline Pionus menstruus (Linnaeus, 1766) & Blue-headed Parrot & $\mathrm{V} * \mathrm{PR}$ & CLO, FMNH, LSUMZ, MUSM, MVZ, UF-FLMNH, UMMZ & CITES-II \\
\hline Graydidascalus brachyurus (Temminck \& Kuhl, 1820) & Short-tailed Parrot & VPR & CLO, FMNH, LSUMZ, MCZ, MUSM, UF-FLMNH, YPM & CITES-II \\
\hline Amazona festiva (Linnaeus, 1758) & Festive Parrot & $V^{*} P R$ & MCZ, MUSM, UMMZ & IUCN-NT, MIN-NT, CITES-II \\
\hline Amazona ochrocephala (Gmelin, JF, 1788) & Yellow-crowned Parrot & $\mathrm{V} * \mathrm{PR}$ & CLO, LSUMZ, MUSM & CITES-II \\
\hline Amazona farinosa (Boddaert, 1783) & Mealy Parrot & V*PR & CLO, FMNH, LSUMZ, MNHN, MUSM, UF-FLMNH & IUCN-NT, CITES-II \\
\hline Amazona amazonica (Linnaeus, 1766) & Orange-winged Parrot & $\mathrm{V} * \mathrm{PR}$ & CLO, FMNH, MUSM & CITES-II \\
\hline Amazona mercenarius (von Tschudi, 1844) & Scaly-naped Parrot & R & & CITES-II \\
\hline Forpus modestus (Cabanis, 1849) & Dusky-billed Parrotlet & $\mathrm{V} * \mathrm{PR}$ & FMNH, LSUMZ, MNHN, MUSM & CITES-II \\
\hline Forpus xanthopterygius (von Spix, 1824) & Blue-winged Parrotlet & $V^{*} P R$ & CLO, CM, FMNH, LSUMZ, MCZ, MIZ, MUSM & CITES-II \\
\hline Pionites melanocephalus (Linnaeus, 1758) & Black-headed Parrot & $\mathrm{V} * \mathrm{PR}$ & CLO, FMNH, LSUMZ, MCZ, MIZ, MNHN, MUSM, UF-FLMNH, USNM, YPM & CITES-II \\
\hline Pionites leucogaster (Kuhl, 1820) & White-bellied Parrot & VPR & FMNH, LSUMZ, MCZ, MUSM, UF-FLMNH, YPM & IUCN-EN, CITES-II \\
\hline Deroptyus accipitrinus (Linnaeus, 1758) & Red-fan Parrot & VP & MUSM & MIN-NT, CITES-II \\
\hline Pyrrhura lucianii (Deville, 1851) & Bonaparte's Parakeet & $\mathrm{V} * \mathrm{PR}$ & RMNH & CITES-II \\
\hline Pyrrhura roseifrons (Gray, GR, 1859) & Rose-fronted Parakeet & VPR & CLO, FMNH, LSUMZ, MUSM & CITES-II \\
\hline Pyrrhura melanura (von Spix, 1824) & Maroon-tailed Parakeet & $V^{*} P R$ & CLO, FMNH, LSUMZ, MCZ, MUSM, UF-FLMNH, YPM & CITES-II \\
\hline Pyrrhura rupicola (von Tschudi, 1844) & Black-capped (Rock) & $\mathrm{PE}$ & & CITES-II \\
\hline Aratinga weddellii (Deville, 1851) & Dusky-headed Parakeet & $\mathrm{V*PR}$ & $A M N H, B M N H, C L O, F M N H, L S U M Z, M C Z$, MNHN, MUSM, UF-FLMNH & CITES-II \\
\hline Orthopsittaca manilatus (Boddaert, 1783) & Red-bellied Macaw & VPR & CLO, MCZ, MUSM & CITES-II \\
\hline Primolius couloni (Sclater, PL, 1876) & Blue-headed Macaw & VPR & MUSM & IUCN-VU, MIN-VU, CITES-I \\
\hline Ara ararauna (Linnaeus, 1758) & Blue-and-yellow Macaw & $\mathrm{V*PR}$ & CLO, MUSM, UF-FLMNH & CITES-II \\
\hline Ara severus (Linnaeus, 1758) & Chestnut-fronted Macaw & $V^{*} P R$ & CLO, MCZ, MNHN, MUSM & CITES-II \\
\hline Ara militaris (Linnaeus, 1766) & Military Macaw & PR & & IUCN-VU, MIN-VU, CITES-I \\
\hline Ara macao (Linnaeus, 1758) & Scarlet Macaw & V*PR & CLO, FMNH, LSUMZ, MUSM & MIN-NT, CITES-I \\
\hline Ara chloropterus Gray, GR, 1859 & Red-and-green Macaw & VPR & CLO, FMNH, MUSM & MIN-NT, CITES-II \\
\hline
\end{tabular}


SALINAS ET AL.

\begin{tabular}{|c|c|c|c|c|}
\hline Nombre científico & Nombre en inglés & Validación & Procedencia de Voucher & Amenaza/endemismo \\
\hline Psittacara leucophthalmus (Statius Muller, 1776) & White-eyed Parakeet & V*PR & CLO, FMNH, LSUMZ, MNHN, MUSM & CITES-II \\
\hline \multicolumn{5}{|l|}{ Orden Passeriformes: Familia Thamnophilidae } \\
\hline Euchrepomis humeralis (Sclater \& Salvin, 1880) & Chestnut-shouldered Antwren & VPR & LSUMZ, MUSM & \\
\hline Euchrepomis spodioptila (Sclater \& Salvin, 1881) & Ash-winged Antwren & VPR & CLO & \\
\hline Cymbilaimus lineatus (Leach, 1814) & Fasciated Antshrike & $\mathrm{V}$ PR & CLO, CM, FMNH, LSUMZ, MUSM, UMMZ & \\
\hline Frederickena unduliger (von Pelzeln, 1868) & Undulated Antshrike & VPR & AMNH, CLO, FMNH, LSUMZ, MUSM & \\
\hline Frederickena fulva Zimmer, JT, 1944 & Fulvous Antshrike & VPR & CLO, LSUMZ & \\
\hline Taraba major (Vieillot, 1816) & Great Antshrike & $\mathrm{V}$ PR & CLO, FMNH, LSUMZ, MCZ, MUSM, MVZ, UF-FLMNH, YPM & \\
\hline Sakesphorus canadensis (Linnaeus, 1766) & Black-crested Antshrike & VPR & FMNH, MUSM & \\
\hline Thamnophilus doliatus (Linnaeus, 1764) & Barred Antshrike & $\mathrm{V} * \mathrm{PR}$ & CLO, FMNH, LSUMZ, MCZ, MUSM, NHMO, UMMZ & \\
\hline Thamnophilus ruficapillus Vieillot, 1816 & Rufous-capped Antshrike & $\mathrm{PE}$ & & \\
\hline Thamnophilus tenuepunctatus Lafresnaye, 1853 & Lined Antshrike & $\mathrm{P}$ & & IUCN-VU \\
\hline Thamnophilus schistaceus d'Orbigny, 1837 & Plain-winged Antshrike & V*PR & CLO, FMNH, KU, LSUMZ, MCZ, MUSM & \\
\hline Thamnophilus murinus Sclater \& Salvin, 1868 & Mouse-colored Antshrike & $\mathrm{V*PR}$ & CLO, FMNH, KU, LSUMZ, MUSM & \\
\hline Thamnophilus praecox Zimmer, JT, 1937 & Cocha Antshrike & $P R$ & & IUCN-NT \\
\hline Thamnophilus cryptoleucus (Menegaux \& Hellmayr, 1906) & Castelnau's Antshrike & VPR & CLO, LSUMZ, MUSM, NHMO, UF-FLMNH & IUCN-NT \\
\hline Thamnophilus caerulescens Vieillot, 1816 & Variable Antshrike & VPR & LSUMZ, MCZ, MUSM & \\
\hline Thamnophilus unicolor (Sclater, PL, 1859) & Uniform Antshrike & $\mathrm{PR}$ & & \\
\hline Thamnophilus aethiops Sclater, PL, 1858 & White-shouldered Antshrike & VPR & CLO, LSUMZ, MUSM & \\
\hline Thamnophilus amazonicus Sclater, PL, 1858 & Amazonian Antshrike & V*PR & CLO, FMNH, LSUMZ, MIZ, MUSM, RMNH & \\
\hline Thamnophilus divisorius Whitney, Oren \& Brumfield, 2004 & Acre Antshrike & $\mathrm{P}$ & & \\
\hline Megastictus margaritatus (Sclater, PL, 1855) & Pearly Antshrike & $\mathrm{V} * \mathrm{PR}$ & FMNH, LSUMZ, MUSM, RMNH, UF-FLMNH & \\
\hline Neoctantes niger (von Pelzeln, 1859) & Black Bushbird & VPR & CLO, FMNH, KU, LSUMZ, MUSM & \\
\hline Thamnistes anabatinus Sclater \& Salvin, 1860 & Russet Antshrike & $\mathrm{P}$ & & \\
\hline Dysithamnus mentalis (Temminck, 1823) & Plain Antvireo & V*P & CLO, LSUMZ, MUSM, UF-FLMNH & \\
\hline Thamnomanes ardesiacus (Sclater \& Salvin, 1868) & Dusky-throated Antshrike & $\mathrm{V*PR}$ & $\mathrm{CLO}, \mathrm{CM}, \mathrm{FMNH}, \mathrm{KU}, \mathrm{LSUMZ}, \mathrm{MUSM}$ & \\
\hline Thamnomanes saturninus (von Pelzeln, 1868) & Saturnine Antshrike & VPR & CLO, FMNH, LSUMZ, MUSM & \\
\hline Thamnomanes caesius (Temminck, 1820) & Cinereous Antshrike & $\mathrm{V} * \mathrm{PR}$ & CLO, CM, FMNH, KU, LSUMZ, MCZ, MUSM, UF-FLMNH, WFVZ & \\
\hline Thamnomanes schistogynus Hellmayr, 1911 & Bluish-slate Antshrike & $\mathrm{VP}$ & CLO, FMNH, LSUMZ, MUSM & \\
\hline Isleria hauxwelli (Sclater, $\mathrm{PL}, 1857$ ) & Plain-throated Antwren & $\mathrm{V*PR}$ & CLO, FMNH, KU, LSUMZ, MCZ, MUSM, ROM, UF-FLMNH & \\
\hline Pygiptila stellaris (von Spix, 1825) & Spot-winged Antshrike & $V^{*} \mathrm{PR}$ & CLO, FMNH, KU, LSUMZ, MCZ, MUSM, ROM & \\
\hline Epinecrophylla leucophthalma (von Pelzeln, 1868) & White-eyed Stipplethroat & VPR & LSUMZ, MUSM & \\
\hline Epinecrophylla haematonota (Sclater, PL, 1857) & Rufous-backed Stipplethroat & V*PR & CLO, FMNH, KU, LSUMZ, MCZ, MUSM & \\
\hline Epinecrophylla spodionota (Sclater \& Salvin, 1880) & Foothill Stipplethroat & $P$ & & \\
\hline Epinecrophylla ornata (Sclater, PL, 1853) & Ornate Stipplethroat & VPR & MCZ & \\
\hline Epinecrophylla erythrura (Sclater, PL, 1890) & Rufous-tailed Stipplethroat & VPR & CLO, FMNH, LSUMZ, MCZ, MUSM & \\
\hline Myrmotherula brachyura (Hermann, 1783) & Pygmy Antwren & $\mathrm{V*PR}$ & CLO, FMNH, LSUMZ, MUSM, UF-FLMNH, UMMZ & \\
\hline Myrmotherula ignota Griscom, 1929 & Moustached Antwren & VPR & AMNH, CLO, FMNH, LSUMZ, MUSM, UF-FLMNH & \\
\hline Myrmotherula sclateri Snethlage, E, 1912 & Sclater's Antwren & $\mathrm{P}$ & & \\
\hline Myrmotherula multostriata Sclater, PL, 1858 & Amazonian Streaked-Antwren & $V * P R$ & CLO, FMNH, LSUMZ, MUSM, UMMZ & \\
\hline
\end{tabular}


LAS AVES DEL DEPARTAMENTO DE LORETO

\begin{tabular}{|c|c|c|c|c|}
\hline Nombre científico & Nombre en inglés & Validación & Procedencia de Voucher & Amenaza/endemismo \\
\hline Myrmotherula cherriei von Berlepsch \& Hartert, 1902 & Cherrie's Antwren & $V P$ & MUSM & \\
\hline Myrmotherula longicauda von Berlepsch \& Stolzmann, 1894 & Stripe-chested Antwren & $\mathrm{P}$ & & \\
\hline Myrmotherula axillaris (Vieillot, 1817) & White-flanked Antwren & $\mathrm{V*PR}$ & CLO, FMNH, KU, LSUMZ, MCZ, MPEG, MUSM, RMNH & \\
\hline Myrmotherula schisticolor (Lawrence, 1865) & Slaty Antwren & VPR & LSUMZ, MUSM & \\
\hline Myrmotherula sunensis Chapman, 1925 & Rio Suno Antwren & $E$ & & \\
\hline Myrmotherula longipennis von Pelzeln, 1868 & Long-winged Antwren & VPR & $\mathrm{CM}, \mathrm{FMNH}, \mathrm{KU}, \mathrm{LSUMZ}, \mathrm{MCZ}$, MUSM & \\
\hline Myrmotherula iheringi Snethlage, E, 1914 & Ihering's Antwren & $\mathrm{PE}$ & & \\
\hline Myrmotherula menetriesii (d'Orbigny, 1837) & Gray Antwren & $\mathrm{V} * \mathrm{PR}$ & CLO, CM, FMNH, LSUMZ, MCZ, MUSM, UMMZ & \\
\hline Myrmotherula assimilis von Pelzeln, 1868 & Leaden Antwren & VPR & CLO, LSUMZ, MUSM, UF-FLMNH & \\
\hline Dichrozona cincta (von Pelzeln, 1868) & Banded Antbird & VPR & CLO, LSUMZ, MUSM & \\
\hline Herpsilochmus parkeri Davis \& O'Neill, 1986 & Ash-throated Antwren & $P R$ & & IUCN-EN, MIN-EN, end \\
\hline Herpsilochmus dugandi Meyer de Schauensee, 1945 & Dugand's Antwren & VPR & CLO, LSUMZ, MUSM, UF-FLMNH & \\
\hline Herpsilochmus gentryi Whitney \& Alvarez-Alonso, 1998 & Ancient Antwren & VPR & LSUMZ, MUSM & MIN-NT \\
\hline Herpsilochmus axillaris (von Tschudi, 1844) & Yellow-breasted Antwren & $P R$ & & IUCN-VU \\
\hline Herpsilochmus rufimarginatus (Temminck, 1822) & Rufous-winged Antwren & $V * P$ & CLO, MUSM & \\
\hline Microrhopias quixensis (Cornalia, 1849) & Dot-winged Antwren & $\mathrm{V} * \mathrm{PR}$ & AMNH, LSUMZ, MUSM & \\
\hline Hypocnemis peruviana Taczanowski, 1884 & Peruvian Warbling-Antbird & $\mathrm{V} * \mathrm{PR}$ & CLO, FMNH, KU, LSUMZ, MCZ, MIZ, MUSM, UF-FLMNH, UMMZ, USNM & \\
\hline Hypocnemis subflava Cabanis, 1873 & Yellow-breasted Warbling-Antbird & VPR & MUSM & \\
\hline Hypocnemis hypoxantha Sclater, PL, 1869 & Yellow-browed Antbird & VPR & CLO, FMNH, KU, LSUMZ, MUSM & \\
\hline Cercomacroides serva (Sclater, PL, 1858) & Black Antbird & $\mathrm{V} * \mathrm{PR}$ & LSUMZ, MUSM & \\
\hline Cercomacroides nigrescens (Cabanis \& Heine, 1860) & Blackish Antbird & $\mathrm{V} * \mathrm{PR}$ & FMNH, LSUMZ, MNHN, MUSM, UMMZ & \\
\hline Cercomacroides fuscicauda Zimmer, JT, 1931 & Riparian Antbird & $P R$ & & \\
\hline Cercomacra cinerascens (Sclater, PL, 1857) & Gray Antbird & $\mathrm{V} * \mathrm{PR}$ & BMNH, CLO, LSUMZ, MCZ, MNHN, MUSM, UF-FLMNH & \\
\hline Pyriglena maura (Ménétries, 1835) & Western Fire-eye & $\mathrm{V} * \mathrm{PR}$ & UMMZ & \\
\hline Myrmoborus leucophrys (von Tschudi, 1844) & White-browed Antbird & VP & LSUMZ & \\
\hline Myrmoborus lugubris (Cabanis, 1847) & Ash-breasted Antbird & $\mathrm{V} * \mathrm{PR}$ & CLO, LSUMZ, MUSM, UF-FLMNH & IUCN-VU \\
\hline Myrmoborus myotherinus (von Spix, 1825) & Black-faced Antbird & $\mathrm{V*PR}$ & AMNH, CLO, CM, FMNH, KU, LSUMZ, MUSM, UF-FLMNH, USNM, WFVZ & \\
\hline Myrmoborus melanurus (Sclater \& Salvin, 1866) & Black-tailed Antbird & VPR & LSUMZ, MUSM & IUCN-VU, MIN-NT \\
\hline Myrmoborus lophotes (Hellmayr \& von Seilern, 1914) & White-lined Antbird & $P R$ & & \\
\hline Hypocnemoides melanopogon (Sclater, PL, 1857) & Black-chinned Antbird & $\mathrm{VP}$ & AMNH, CLO, LSUMZ, MUSM, UF-FLMNH & \\
\hline Hypocnemoides maculicauda (von Pelzeln, 1868) & Band-tailed Antbird & $\mathrm{VP}$ & CLO, FMNH, LSUMZ, MUSM & \\
\hline Myrmochanes hemileucus (Sclater \& Salvin, 1866) & Black-and-white Antbird & $\mathrm{VP}$ & CLO, FMNH, LSUMZ, MUSM, NHMO, UF-FLMNH & \\
\hline Sclateria naevia (Gmelin, JF, 1788) & Silvered Antbird & $\mathrm{V} * \mathrm{PR}$ & CLO, FMNH, LSUMZ, MUSM, UF-FLMNH & \\
\hline Percnostola rufifrons (Gmelin, JF, 1789) & Black-headed Antbird & $\mathrm{VP}$ & LSUMZ & \\
\hline Percnostola arenarum Isler, Alvarez, Isler \& Whitney, 2002 & Allpahuayo Antbird & VPR & LSUMZ, MUSM & IUCN-VU, MIN-VU, end \\
\hline Myrmelastes schistaceus (Sclater, PL, 1858) & Slate-colored Antbird & VPR & CLO, LSUMZ, MUSM & \\
\hline Myrmelastes hyperythrus (Sclater, PL, 1855) & Plumbeous Antbird & $\mathrm{V} * \mathrm{PR}$ & CLO, FMNH, LSUMZ, MUSM, UF-FLMNH & \\
\hline Myrmelastes leucostigma (von Pelzeln, 1868) & Spot-winged Antbird & VPR & CLO, LSUMZ, MUSM, UF-FLMNH & \\
\hline Sciaphylax hemimelaena (Sclater, PL, 1857) & Chestnut-tailed Antbird & VPR & CLO, FMNH, LSUMZ, MUSM & \\
\hline Sciaphylax castanea (Zimmer, JT, 1932) & Zimmer's Antbird & VPR & LSUMZ, MUSM & \\
\hline
\end{tabular}


SALINAS ET AL.

\begin{tabular}{|c|c|c|c|c|}
\hline Nombre científico & Nombre en inglés & Validación & Procedencia de Voucher & Amenaza/endemismo \\
\hline Akletos melanoceps (von Spix, 1825) & White-shouldered Antbird & VPR & CLO, CM, FMNH, KU, LSUMZ, MUSM & \\
\hline Hafferia fortis (Sclater \& Salvin, 1868) & Sooty Antbird & V*PR & CLO, KU, LSUMZ, MUSM, UF-FLMNH & \\
\hline Myrmophylax atrothorax (Boddaert, 1783) & Black-throated Antbird & $V * P R$ & AMNH, CLO, FMNH, LSUMZ, MIZ, MUSM, UF-FLMNH & \\
\hline Myrmornis torquata (Boddaert, 1783) & Wing-banded Antbird & VPR & LSUMZ & \\
\hline Pithys albifrons (Linnaeus, 1766) & White-plumed Antbird & V*PR & CLO, KU, LSUMZ, MUSM & \\
\hline Pithys castaneus Berlioz, 1938 & White-masked Antbird & VPR & LSUMZ, MUSM & IUCN-NT, MIN-VU, end \\
\hline Gymnopithys leucaspis (Sclater, PL, 1855) & White-cheeked Antbird & V*PR & AMNH, CLO, KU, LSUMZ, MUSM & \\
\hline Oneillornis salvini (von Berlepsch, 1901) & White-throated Antbird & VPR & FMNH, LSUMZ, MUSM & \\
\hline Oneillornis lunulatus (Sclater \& Salvin, 1873) & Lunulated Antbird & VPR & CLO, LSUMZ, MUSM & \\
\hline Rhegmatorhina melanosticta (Sclater \& Salvin, 1880) & Hairy-crested Antbird & VPR & CLO, FMNH, KU, LSUMZ, MUSM & \\
\hline Hylophylax naevius (Gmelin, JF, 1789) & Spot-backed Antbird & V*PR & CLO, KU, LSUMZ, MUSM, RMNH, UF-FLMNH & \\
\hline Hylophylax punctulatus (Des Murs, 1856) & Dot-backed Antbird & VPR & CLO, FMNH, LSUMZ, MUSM & \\
\hline Willisornis poecilinotus (Cabanis, 1847) & Common Scale-backed Antbird & $V * P R$ & CLO, FMNH, KU, LSUMZ, MUSM, RMNH, UF-FLMNH & \\
\hline Phlegopsis nigromaculata (d'Orbigny \& Lafresnaye, 1837) & Black-spotted Bare-eye & $\mathrm{V} * \mathrm{PR}$ & CLO, CM, FMNH, KU, LSUMZ, MUSM, UF-FLMNH, UMMZ, YPM & \\
\hline Phlegopsis erythroptera (Gould, 1855) & Reddish-winged Bare-eye & VPR & CLO, KU, LSUMZ, MUSM & \\
\hline \multicolumn{5}{|l|}{ Orden Passeriformes: Familia Conopophagidae } \\
\hline Conopophaga aurita (Gmelin, JF, 1789) & Chestnut-belted Gnateater & VP & CLO, FMNH, LSUMZ, MCZ, MUSM & \\
\hline Conopophaga peruviana Des Murs, 1856 & Ash-throated Gnateater & V*PR & CLO, KU, LSUMZ, MUSM & \\
\hline Conopophaga castaneiceps Sclater, PL, 1857 & Chestnut-crowned Gnateater & VP & LSUMZ, MUSM & \\
\hline \multicolumn{5}{|l|}{ Orden Passeriformes: Familia Grallariidae } \\
\hline Grallaria squamigera Lafresnaye, 1842 & Undulated Antpitta & $\mathrm{V} * \mathrm{PE}$ & MCZ & \\
\hline Grallaria varia (Boddaert, 1783) & Variegated Antpitta & VP & CLO, LSUMZ & \\
\hline Grallaria guatimalensis Lafresnaye, 1842 & Scaled Antpitta & VP & CLO, MUSM & \\
\hline Grallaria haplonota Sclater, PL, 1877 & Plain-backed Antpitta & PR & & \\
\hline Grallaria dignissima Sclater \& Salvin, 1880 & Ochre-striped Antpitta & VPR & CLO, YPM & \\
\hline Grallaria eludens Lowery \& O'Neill, 1969 & Elusive Antpitta & VP & MUSM & MIN-NT \\
\hline Grallaria carrikeri Schulenberg \& Williams, 1982 & Pale-billed Antpitta & $\mathrm{PE}$ & & IUCN-NT, MIN-NT, end \\
\hline Hylopezus macularius (Temminck, 1830) & Spotted Antpitta & VP & AMNH & \\
\hline Myrmothera fulviventris (Sclater, PL, 1858) & White-lored Antpitta & VPR & CLO, LSUMZ, MUSM, UF-FLMNH & \\
\hline Myrmothera berlepschi (Hellmayr, 1903) & Amazonian Antpitta & VP & CLO & \\
\hline Myrmothera campanisona (Hermann, 1783) & Thrush-like Antpitta & V*PR & CLO, FMNH, LSUMZ, MIZ, MUSM, UF-FLMNH & \\
\hline \multicolumn{5}{|l|}{ Orden Passeriformes: Familia Rhinocryptidae } \\
\hline Liosceles thoracicus (Sclater, PL, 1865) & Rusty-belted Tapaculo & VPR & CLO, LSUMZ, MUSM, WFVZ & \\
\hline Scytalopus femoralis (von Tschudi, 1844) & Rufous-vented Tapaculo & VR & CLO, MUSM & end \\
\hline Scytalopus atratus Hellmayr, 1922 & White-crowned Tapaculo & VP & CLO, LSUMZ, MUSM & \\
\hline \multicolumn{5}{|l|}{ Orden Passeriformes: Familia Formicariidae } \\
\hline Formicarius colma Boddaert, 1783 & Rufous-capped Antthrush & V*PR & CLO, FMNH, KU, LSUMZ, MCZ, MUSM, UF-FLMNH & \\
\hline Formicarius analis (d'Orbigny \& Lafresnaye, 1837) & Black-faced Antthrush & V*PR & CLO, FMNH, LSUMZ, MCZ, MUSM, UF-FLMNH & \\
\hline Formicarius rufipectus Salvin, 1866 & Rufous-breasted Antthrush & $P$ & & \\
\hline Chamaeza campanisona (Lichtenstein, MHC, 1823) & Short-tailed Antthrush & VPR & LSUMZ, MUSM & \\
\hline
\end{tabular}


LAS AVES DEL DEPARTAMENTO DE LORETO

\begin{tabular}{|c|c|c|c|c|}
\hline Nombre científico & Nombre en inglés & Validación & Procedencia de Voucher & Amenaza/endemismo \\
\hline Chamaeza nobilis Gould, 1855 & Striated Antthrush & VPR & CLO, FMNH, LSUMZ, MUSM & \\
\hline \multicolumn{5}{|l|}{ Orden Passeriformes: Familia Furnariidae } \\
\hline Sclerurus obscurior Sclater, PL, 1857 & Tawny-throated Leaftosser & VPR & CLO, FMNH, KU, LSUMZ, MUSM & \\
\hline Sclerurus rufigularis von Pelzeln, 1868 & Short-billed Leaftosser & VPR & CLO, LSUMZ, MUSM & \\
\hline Sclerurus caudacutus (Vieillot, 1816) & Black-tailed Leaftosser & $\mathrm{V} * \mathrm{PR}$ & CLO, LSUMZ, MUSM & \\
\hline Sclerurus albigularis Sclater \& Salvin, 1869 & Gray-throated Leaftosser & VP & LSUMZ, MUSM & IUCN-NT \\
\hline Certhiasomus stictolaemus (von Pelzeln, 1868) & Spot-throated Woodcreeper & $\mathrm{V} * \mathrm{PR}$ & KU, LSUMZ, MUSM, UF-FLMNH & \\
\hline Sittasomus griseicapillus (Vieillot, 1818) & Olivaceous Woodcreeper & $\mathrm{V} * \mathrm{PR}$ & CM, FMNH, LSUMZ, MUSM, UF-FLMNH & \\
\hline Deconychura longicauda (von Pelzeln, 1868) & Long-tailed Woodcreeper & VPR & CLO, FMNH, KU, LSUMZ, MUSM & \\
\hline Dendrocincla tyrannina (Lafresnaye, 1851) & Tyrannine Woodcreeper & $\mathrm{R}$ & & \\
\hline Dendrocincla merula (Lichtenstein, MHC, 1820) & White-chinned Woodcreeper & $\mathrm{V} * \mathrm{PR}$ & CLO, FMNH, LSUMZ, MUSM, UF-FLMNH & \\
\hline Dendrocincla fuliginosa (Vieillot, 1818) & Plain-brown Woodcreeper & VPR & CLO, CM, FMNH, KU, LSUMZ, MUSM & \\
\hline Glyphorynchus spirurus (Vieillot, 1819) & Wedge-billed Woodcreeper & V*PR & CLO, CM, FMNH, KU, LSUMZ, MUSM, RMNH, ROM, UF-FLMNH, YPM & \\
\hline Dendrexetastes rufigula (Lesson, 1844) & Cinnamon-throated Woodcreeper & VPR & CLO, CM, FMNH, LSUMZ, MUSM & \\
\hline Nasica longirostris (Vieillot, 1818) & Long-billed Woodcreeper & VPR & CLO, CM, FMNH, LSUMZ, MCZ, MUSM & \\
\hline Dendrocolaptes certhia (Boddaert, 1783) & Amazonian Barred-Woodcreeper & $\mathrm{V} * \mathrm{PR}$ & $\mathrm{CLO}, \mathrm{CM}, \mathrm{FMNH}, \mathrm{KU}, \mathrm{LSUMZ}, \mathrm{MCZ}$, MNHN, MUSM, UF-FLMNH & \\
\hline Dendrocolaptes picumnus Lichtenstein, MHC, 1820 & Black-banded Woodcreeper & $\mathrm{V} * \mathrm{PR}$ & LSUMZ, FMNH, MNHN, MUSM & \\
\hline Hylexetastes stresemanni Snethlage, E, 1925 & Bar-bellied Woodcreeper & $\mathrm{VP}$ & MUSM & \\
\hline Xiphocolaptes promeropirhynchus (Lesson, 1840) & Strong-billed Woodcreeper & $\mathrm{VP}$ & CLO, FMNH, LSUMZ, MUSM, ROM, UF-FLMNH & \\
\hline Xiphorhynchus obsoletus (Lichtenstein, MHC, 1820) & Striped Woodcreeper & $\mathrm{VP}$ & CLO, FMNH, LSUMZ, MUSM, UF-FLMNH & \\
\hline Xiphorhynchus ocellatus (von Spix, 1824) & Ocellated Woodcreeper & VPR & AMNH, FMNH, KU, LSUMZ, MCZ, MUSM, UF-FLMNH & \\
\hline Xiphorhynchus elegans (von Pelzeln, 1868) & Elegant Woodcreeper & VPR & AMNH, CLO, CM, FMNH, LSUMZ, MUSM & \\
\hline Xiphorhynchus guttatus (Lichtenstein, MHC, 1820) & Buff-throated Woodcreeper & VPR & CLO, FMNH, LSUMZ, MCZ, MUSM, UF-FLMNH & \\
\hline Xiphorhynchus triangularis (Lafresnaye, 1842) & Olive-backed Woodcreeper & $\mathrm{VP}$ & FMNH & \\
\hline Dendroplex picus (Gmelin, JF, 1788) & Straight-billed Woodcreeper & VPR & CLO, CM, FMNH, LSUMZ, MUSM, YPM & \\
\hline Dendroplex kienerii Des Murs, 1856 & Zimmer's Woodcreeper & VPR & CM, LSUMZ, MUSM & IUCN-NT \\
\hline Campylorhamphus trochilirostris (Lichtenstein, MHC, 1820) & Red-billed Scythebill & VPR & LSUMZ, MUSM & \\
\hline Campylorhamphus procurvoides (Lafresnaye, 1850) & Curve-billed Scythebill & $\mathrm{VP}$ & CLO, LSUMZ & \\
\hline Campylorhamphus pusillus (Sclater, PL, 1860) & Brown-billed Scythebill & $\mathrm{v}$ & LSUMZ & \\
\hline Lepidocolaptes duidae Zimmer, JT, 1934 & Duida Woodcreeper & VPR & CLO, FMNH, LSUMZ, MUSM & \\
\hline $\begin{array}{l}\text { Lepidocolaptes fatimalimae Rodrigues, Aleixo, Whittaker \& Naka, } \\
2013\end{array}$ & Inambari Woodcreeper & VPR & CLO & \\
\hline Xenops tenuirostris von Pelzeln, 1859 & Slender-billed Xenops & VPR & LSUMZ, UMMZ & \\
\hline Xenops minutus (Sparrman, 1788) & Plain Xenops & $\mathrm{V} * \mathrm{PR}$ & FMNH, LSUMZ, MUSM, UF-FLMNH & \\
\hline Xenops rutilans Temminck, 1821 & Streaked Xenops & PR & & \\
\hline Berlepschia rikeri (Ridgway, 1887) & Point-tailed Palmcreeper & VPR & LSUMZ & \\
\hline Microxenops milleri Chapman, 1914 & Rufous-tailed Xenops & VPR & CLO, LSUMZ, MCZ, MUSM & \\
\hline Pseudocolaptes boissonneautii (Lafresnaye, 1840) & Streaked Tuftedcheek & VPR & LSUMZ, MUSM & \\
\hline Furnarius leucopus Swainson, 1838 & Pale-legged Hornero & VPR & CLO, CM, FMNH, LSUMZ, MUSM & \\
\hline Furnarius torridus Sclater \& Salvin, 1866 & Pale-billed Hornero & $\mathrm{V} * \mathrm{p}$ & CLO, CM, FMNH, LSUMZ, MCZ, MUSM, UF-FLMNH & \\
\hline Furnarius minor von Pelzeln, 1858 & Lesser Hornero & $\mathrm{V} * \mathrm{P}$ & CLO, CM, LSUMZ, MUSM, NHMO, UF-FLMNH & \\
\hline
\end{tabular}


SALINAS ET AL.

\begin{tabular}{|c|c|c|c|c|}
\hline Nombre científico & Nombre en inglés & Validación & Procedencia de Voucher & Amenaza/endemismo \\
\hline Lochmias nematura (Lichtenstein, MHC, 1823) & Sharp-tailed Streamcreeper & PE & & \\
\hline Anabazenops dorsalis (Sclater \& Salvin, 1880) & Dusky-cheeked Foliage-gleaner & $\mathrm{P}$ & & \\
\hline Philydor erythrocercum (von Pelzeln, 1859) & Rufous-rumped Foliage-gleaner & VPR & CLO, FMNH, LSUMZ, MUSM & \\
\hline Philydor pyrrhodes (Cabanis, 1849) & Cinnamon-rumped Foliage-gleaner & V*PR & CLO, FMNH, LSUMZ, MUSM & \\
\hline Anabacerthia striaticollis Lafresnaye, 1840 & Montane Foliage-gleaner & $R$ & & \\
\hline Anabacerthia ruficaudata (d'Orbigny \& Lafresnaye, 1838) & Rufous-tailed Foliage-gleaner & VPR & LSUMZ, MCZ, MUSM & \\
\hline Syndactyla rufosuperciliata (Lafresnaye, 1832) & Buff-browed Foliage-gleaner & VPR & LSUMZ, MUSM & \\
\hline Syndactyla subalaris (Sclater, PL, 1859) & Lineated Foliage-gleaner & $\mathrm{VP}$ & LSUMZ, MUSM & \\
\hline Ancistrops strigilatus (von Spix, 1825) & Chestnut-winged Hookbill & V*PR & CLO, KU, LSUMZ, MCZ, MUSM, UF-FLMNH & \\
\hline Dendroma rufa (Vieillot, 1818) & Buff-fronted Foliage-gleaner & VPR & LSUMZ & \\
\hline Dendroma erythroptera (Sclater, PL, 1856) & Chestnut-winged Foliage-gleaner & $\mathrm{V}$ PR & CLO, LSUMZ, MNHN, MUSM & \\
\hline Clibanornis rubiginosus (Sclater, PL, 1857) & Ruddy Foliage-gleaner & VPR & CLO, LSUMZ, MUSM, UF-FLMNH & \\
\hline Thripadectes melanorhynchus (von Tschudi, 1844) & Black-billed Treehunter & $\mathrm{VP}$ & LSUMZ & \\
\hline Automolus rufipileatus (von Pelzeln, 1859) & Chestnut-crowned Foliage-gleaner & VPR & CLO, LSUMZ, MCZ, MUSM, UF-FLMNH & \\
\hline Automolus melanopezus (Sclater, PL, 1858) & Brown-rumped Foliage-gleaner & VPR & MUSM & \\
\hline Automolus ochrolaemus (von Tschudi, 1844) & Buff-throated Foliage-gleaner & VPR & CLO, FMNH, KU, LSUMZ, MUSM & \\
\hline Automolus subulatus (von Spix, 1824) & Striped Woodhaunter & V*PR & CLO, FMNH, KU, LSUMZ, MUSM & \\
\hline Automolus infuscatus (Sclater, PL, 1856) & Olive-backed Foliage-gleaner & $\mathrm{V}$ PR & CM, CLO, FMNH, KU, LSUMZ, MCZ, MUSM, UF-FLMNH & \\
\hline Premnoplex brunnescens (Sclater, PL, 1856) & Spotted Barbtail & $\mathrm{VP}$ & LSUMZ, MUSM & \\
\hline Metopothrix aurantiaca Sclater \& Salvin, 1866 & Orange-fronted Plushcrown & VPR & CLO, LSUMZ, MUSM, UF-FLMNH & \\
\hline Thripophaga fusciceps Sclater, PL, 1889 & Plain Softtail & $P$ & & \\
\hline Cranioleuca vulpina (von Pelzeln, 1856) & Rusty-backed Spinetail & V*P & MUSM, UMMZ & \\
\hline Cranioleuca vulpecula (Sclater \& Salvin, 1866) & Parker's Spinetail & VPR & CLO, LSUMZ, MUSM, NHMO & \\
\hline Cranioleuca curtata (Sclater, PL, 1870) & Ash-browed Spinetail & $R$ & & IUCN-VU \\
\hline Cranioleuca gutturata (d'Orbigny \& Lafresnaye, 1838) & Speckled Spinetail & V*PR & CLO, FMNH, KU, LSUMZ, MUSM, UF-FLMNH & \\
\hline Certhiaxis cinnamomeus (Gmelin, JF, 1788) & Yellow-chinned Spinetail & $\mathrm{PR}$ & & \\
\hline Certhiaxis mustelinus (Sclater, PL, 1874) & Red-and-white Spinetail & V*P & CLO, LSUMZ, MNHN, MUSM, UMMZ & \\
\hline Mazaria propinqua von Pelzeln, 1859 & White-bellied Spinetail & VPR & CLO, LSUMZ, MUSM, NHMO, UF-FLMNH & \\
\hline Synallaxis gujanensis (Gmelin, JF, 1789) & Plain-crowned Spinetail & VPR & CLO, CM, FMNH, LSUMZ, MCZ, MUSM & \\
\hline Synallaxis moesta Sclater, PL, 1856 & Dusky Spinetail & $\mathrm{VP}$ & UMMZ & IUCN-NT \\
\hline Synallaxis albigularis Sclater, PL, 1858 & Dark-breasted Spinetail & VPR & CLO, CM, FMNH, LSUMZ, MCZ, MUSM, UF-FLMNH & \\
\hline Synallaxis albescens Temminck, 1823 & Pale-breasted Spinetail & V*P & MUSM & \\
\hline Synallaxis azarae d'Orbigny, 1835 & Azara's Spinetail & $R$ & & \\
\hline Synallaxis rutilans Temminck, 1823 & Ruddy Spinetail & V*PR & CLO, KU, LSUMZ, MCZ, MUSM, NHMO, RMNH, UF-FLMNH & \\
\hline Synallaxis cherriei Gyldenstolpe, 1930 & Chestnut-throated Spinetail & $\mathrm{P}$ & & IUCN-NT, MIN-NT \\
\hline \multicolumn{5}{|l|}{ Orden Passeriformes: Familia Pipridae } \\
\hline Tyranneutes stolzmanni (Hellmayr, 1906) & Dwarf Tyrant-Manakin & VPR & CLO, LSUMZ, MUSM, UF-FLMNH & \\
\hline Neopelma chrysocephalum (von Pelzeln, 1868) & Saffron-crested Tyrant-Manakin & $\mathrm{VP}$ & CLO, MUSM & MIN-VU \\
\hline Neopelma sulphureiventer (Hellmayr, 1903) & Sulphur-bellied Tyrant-Manakin & $P R$ & & \\
\hline Chloropipo unicolor Taczanowski, 1884 & Jet Manakin & VPR & LSUMZ, MUSM & \\
\hline
\end{tabular}


LAS AVES DEL DEPARTAMENTO DE LORETO

\begin{tabular}{|c|c|c|c|c|}
\hline Nombre científico & Nombre en inglés & Validación & Procedencia de Voucher & Amenaza/endemismo \\
\hline Chiroxiphia pareola (Linnaeus, 1766) & Blue-backed Manakin & VPR & $\mathrm{CLO}, \mathrm{CM}, \mathrm{FMNH}, \mathrm{KU}, \mathrm{LSUMZ}, \mathrm{ROM}, \mathrm{UF}-\mathrm{FLMNH}$ & \\
\hline Masius chrysopterus (Lafresnaye, 1843) & Golden-winged Manakin & $\mathrm{VP}$ & LSUMZ & \\
\hline Xenopipo atronitens Cabanis, 1847 & Black Manakin & VPR & LSUMZ, MUSM & \\
\hline Cryptopipo holochlora (Sclater, PL, 1888) & Green Manakin & VPR & KU, LSUMZ, MUSM & \\
\hline Lepidothrix coronata (von Spix, 1825) & Blue-crowned Manakin & $\mathrm{V*PR}$ & $\begin{array}{l}\text { AMNHH, CCO, CM, FMNH, KU, LSUMZ, MPEG, MUSM, MVZ, ROM, UF- } \\
\text { FLMNH, USNM, YPM }\end{array}$ & \\
\hline Lepidothrix isidorei (Sclater, PL, 1852) & Blue-rumped Manakin & $P R$ & & IUCN-NT \\
\hline Lepidothrix coeruleocapilla (von Tschudi, 1844) & Cerulean-capped Manakin & $\mathrm{VP}$ & MUSM & end \\
\hline Heterocercus aurantiivertex Sclater \& Salvin, 1880 & Orange-crowned Manakin & VPR & LSUMZ, MUSM & \\
\hline Manacus manacus (Linnaeus, 1766) & White-bearded Manakin & $\mathrm{V} * \mathrm{PR}$ & CLO, FMNH, LSUMZ, MUSM, USNM & \\
\hline Pipra filicauda von Spix, 1825 & Wire-tailed Manakin & $\mathrm{V} * \mathrm{PR}$ & FMNH, KU, LSUMZ, MCZ, MUSM, RMNH, UF-FLMNH, UMMZ, YPM & \\
\hline Pipra fasciicauda Hellmayr, 1906 & Band-tailed Manakin & $\mathrm{VP}$ & CLO, LSUMZ, MUSM, UF-FLMNH & \\
\hline Machaeropterus striolatus (Hahn, 1819) & Striolated Manakin & $\mathrm{V} * \mathrm{PR}$ & $\begin{array}{l}\text { AMNH, COO, FMNH, KU, LSUMZ, MCZ, MUSM, ROM, UF-FLMNH, UMMZ, } \\
\text { USNM, YPM }\end{array}$ & \\
\hline Machaeropterus eckelberryi Lane, Kratter \& O'Neill, 2017 & Painted Manakin & $\mathrm{VP}$ & MUSM & end \\
\hline Machaeropterus pyrocephalus (Sclater, PL, 1852) & Fiery-capped Manakin & $\mathrm{PR}$ & & \\
\hline Pseudopipra pipra (Linnaeus, 1758) & White-crowned Manakin & V*PR & $\begin{array}{l}\text { CLO, FMNH, KÜ, LSUMZZ, MCZ, MPEG, MUUSM, ROM, UF-FLMNH, UMMZ, } \\
\text { WFVZ, YPM }\end{array}$ & \\
\hline Ceratopipra erythrocephala (Linnaeus, 1758) & Golden-headed Manakin & $\mathrm{V} * \mathrm{PR}$ & CLO, CM, FMNH, KU, LSUMZ, MUSM, UF-FLMNH, USNM, YPM & \\
\hline Ceratopipra rubrocapilla (Temminck, 1821) & Red-headed Manakin & VPR & FMNH, LSUMZ, MCZ, MUSM, YPM & \\
\hline Ceratopipra chloromeros (von Tschudi, 1844) & Round-tailed Manakin & VPR & LSUMZ, MUSM & \\
\hline \multicolumn{5}{|l|}{ Orden Passeriformes: Familia Cotingidae } \\
\hline Pipreola frontalis (Sclater, PL, 1859) & Scarlet-breasted Fruiteater & $\mathrm{VP}$ & LSUMZ, MUSM & \\
\hline Pipreola chlorolepidota Swainson, 1838 & Fiery-throated Fruiteater & VP & LSUMZ, MUSM & IUCN-NT, MIN-NT \\
\hline Ampelioides tschudii (Gray, GR, 1846) & Scaled Fruiteater & $P$ & & \\
\hline Ampelion rufaxilla (von Tschudi, 1844) & Chestnut-crested Cotinga & $\mathrm{VP}$ & LSUMZ, MUSM & \\
\hline Phoenicircus nigricollis Swainson, 1832 & Black-necked Red-Cotinga & $\mathrm{V} * \mathrm{PR}$ & CAS, CLO, LSUMZ, MNHN, MUSM, UF-FLMNH, UMMZ, USNM & \\
\hline Rupicola peruvianus (Latham, 1790) & Andean Cock-of-the-rock & $\mathrm{VP}$ & DMNH, LSUMZ, UMMZ & CITES-II \\
\hline Snowornis subalaris (Sclater, PL, 1861) & Gray-tailed Piha & $\mathrm{VP}$ & LSUMZ, MUSM & \\
\hline Snowornis cryptolophus (Sclater \& Salvin, 1877) & Olivaceous Piha & VP & CLO, LSUMZ, MUSM & \\
\hline Querula purpurata (Statius Muller, 1776) & Purple-throated Fruitcrow & VPR & CLO, FMNH, KU, LSUMZ, MUSM, UF-FLMNH & \\
\hline Cephalopterus ornatus Geoffroy Saint-Hilaire, E, 1809 & Amazonian Umbrellabird & $\mathrm{V} * \mathrm{PR}$ & FMNH, LSUMZ, RMNH & CITES-III \\
\hline Cotinga maynana (Linnaeus, 1766) & Plum-throated Cotinga & $\mathrm{V}^{*} \mathrm{PR}$ & FMNH, LSUMZ, MUSM, YPM & \\
\hline Cotinga cotinga (Linnaeus, 1766) & Purple-breasted Cotinga & $\mathrm{VP}$ & LSUMZ, MUSM & \\
\hline Cotinga cayana (Linnaeus, 1766) & Spangled Cotinga & VPR & FMNH, LSUMZ, MUSM & \\
\hline Lipaugus vociferans (zu Wied-Neuwied, 1820) & Screaming Piha & VPR & CLO, FMNH, KU, LSUMZ, MCZ, MUSM, UF-FLMNH & \\
\hline Porphyrolaema porphyrolaema (Deville \& Sclater, 1852) & Purple-throated Cotinga & VPR & CLO, FMNH, LSUMZ, MUSM, USNM & \\
\hline Xipholena punicea (Pallas, 1764) & Pompadour Cotinga & $\mathrm{VP}$ & LSUMZ, MUSM & $\mathrm{MIN}-\mathrm{VU}$ \\
\hline Gymnoderus foetidus (Linnaeus, 1758) & Bare-necked Fruitcrow & VPR & FMNH, LSUMZ, MUSM, UMMZ & \\
\hline \multicolumn{5}{|l|}{ Orden Passeriformes: Familia Tityridae } \\
\hline Tityra inquisitor (Lichtenstein, MHC, 1823) & Black-crowned Tityra & VPR & LSUMZ, MUSM & \\
\hline Tityra cayana (Linnaeus, 1766) & Black-tailed Tityra & VPR & CLO, LSUMZ, MUSM, UMMZ & \\
\hline
\end{tabular}


SALINAS ET AL.

\begin{tabular}{|c|c|c|c|c|}
\hline Nombre científico & Nombre en inglés & Validación & Procedencia de Voucher & Amenaza/endemismo \\
\hline Tityra semifasciata (von Spix, 1825) & Masked Tityra & $\mathrm{V}$ PR & LSUMZ, MUSM, YPM & \\
\hline Schiffornis major Des Murs, 1856 & Varzea Schiffornis & VPR & CLO, FMNH, LSUMZ, MUSM, UF-FLMNH & \\
\hline Schiffornis aenea Zimmer, JT, 1936 & Foothill Schiffornis & $\mathrm{P}$ & & \\
\hline Schiffornis turdina (zu Wied, 1831) & Brown-wing Schiffornis & VPR & LSUMZ, MUSM & \\
\hline Laniocera hypopyrra (Vieillot, 1817) & Cinereous Mourner & VPR & CLO, LSUMZ, MUSM, UF-FLMNH & \\
\hline lodopleura isabellae Parzudaki, 1847 & White-browed Purpletuft & VPR & CLO, FMNH, LSUMZ, MUSM, USNM & \\
\hline Laniisoma elegans (Thunberg, 1823) & Elegant Mourner & $P$ & & IUCN-NT \\
\hline Pachyramphus viridis (Vieillot, 1816) & Green-backed Becard & $\mathrm{P}$ & & \\
\hline Pachyramphus versicolor (Hartlaub, 1843) & Barred Becard & $\mathrm{VP}$ & LSUMZ & \\
\hline Pachyramphus rufus (Boddaert, 1783) & Cinereous Becard & VP & CLO, LSUMZ & \\
\hline Pachyramphus castaneus (Jardine \& Selby, 1827) & Chestnut-crowned Becard & VPR & CLO, FMNH, LSUMZ, MUSM & \\
\hline Pachyramphus polychopterus (Vieillot, 1818) & White-winged Becard & VPR & CLO, FMNH, LSUMZ, NHMO, MCZ, MUSM, YPM & \\
\hline Pachyramphus albogriseus Sclater, PL, 1857 & Black-and-white Becard & $P R$ & & \\
\hline Pachyramphus marginatus (Lichtenstein, MHC, 1823) & Black-capped Becard & VPR & CLO, LSUMZ, MCZ, MUSM & \\
\hline Pachyramphus minor (Lesson, 1831) & Pink-throated Becard & VPR & CLO, LSUMZ, MUSM & \\
\hline \multicolumn{5}{|l|}{ Orden Passeriformes: Familia Oxyruncidae } \\
\hline Oxyruncus cristatus Swainson, 1821 & Sharpbill & $\mathrm{VP}$ & LSUMZ, MUSM & \\
\hline \multicolumn{5}{|l|}{ Orden Passeriformes: Familia Onychorhynchidae } \\
\hline Onychorhynchus coronatus (Statius Muller, 1776) & Royal Flycatcher & VPR & AMNH, CLO, FMNH, LSUMZ, MUSM, UF-FLMNH & MIN-VU \\
\hline Terenotriccus erythrurus (Cabanis, 1847) & Ruddy-tailed Flycatcher & $\mathrm{V} * \mathrm{PR}$ & CLO, FMNH, LSUMZ, MCZ, MUSM, UF-FLMNH & \\
\hline Myiobius villosus Sclater, PL, 1860 & Tawny-breasted Flycatcher & $\mathrm{P}$ & & \\
\hline Myiobius barbatus (Gmelin, JF, 1789) & Sulphur-rumped Flycatcher & V*PR & KU, LSUMZ, MUSM, RMNH & \\
\hline Myiobius atricaudus Lawrence, 1863 & Black-tailed Flycatcher & VPR & CM, FMNH, LSUMZ, MCZ, MUSM & \\
\hline \multicolumn{5}{|l|}{ Orden Passeriformes: Familia Tyrannidae } \\
\hline Piprites chloris (Temminck, 1822) & Wing-barred Piprites & VPR & CLO, KU, LSUMZ, MUSM & \\
\hline Neopipo cinnamomea (Lawrence, 1869) & Cinnamon Manakin-Tyrant & VPR & LSUMZ, MUSM & \\
\hline Platyrinchus saturatus Salvin \& Godman, 1882 & Cinnamon-crested Spadebill & $\mathrm{VP}$ & MUSM & \\
\hline Platyrinchus mystaceus Vieillot, 1818 & White-throated Spadebill & $\mathrm{VP}$ & LSUMZ, MUSM & \\
\hline Platyrinchus coronatus Sclater, PL, 1858 & Golden-crowned Spadebill & VPR & CLO, LSUMZ, MUSM & \\
\hline Platyrinchus flavigularis Sclater, PL, 1862 & Yellow-throated Spadebill & $\mathrm{VP}$ & LSUMZ, MUSM & \\
\hline Platyrinchus platyrhynchos (Gmelin, JF, 1788) & White-crested Spadebill & VPR & CLO, LSUMZ, MUSM & \\
\hline Corythopis torquatus von Tschudi, 1844 & Ringed Antpipit & VPR & CLO, KU, LSUMZ, MUSM, UF-FLMNH & \\
\hline Phylloscartes ophthalmicus Taczanowski, 1874 & Marble-faced Bristle-Tyrant & $P R$ & & \\
\hline Phylloscartes orbitalis (Cabanis, 1873) & Spectacled Bristle-Tyrant & VPR & MCZ & \\
\hline Phylloscartes ventralis (Temminck, 1824) & Mottle-cheeked Tyrannulet & $\mathrm{VP}$ & CLO, LSUMZ, MUSM & \\
\hline Mionectes striaticollis (d'Orbigny \& Lafresnaye, 1837) & Streak-necked Flycatcher & $\mathrm{VP}$ & LSUMZ, MUSM & \\
\hline Mionectes olivaceus Lawrence, 1868 & Olive-striped Flycatcher & VP & LSUMZ, MUSM & \\
\hline Mionectes oleagineus (Lichtenstein, MHC, 1823) & Ochre-bellied Flycatcher & $\mathrm{V} * \mathrm{PR}$ & CLO, FMNH, KU, LSUMZ, MIZ, MUSM, UF-FLMNH, USNM & \\
\hline Mionectes macconnelli (Chubb, C, 1919) & McConnell's Flycatcher & $\mathrm{P}$ & & \\
\hline Leptopogon amaurocephalus von Tschudi, 1846 & Sepia-capped Flycatcher & VPR & LSUMZ, MUSM, YPM & \\
\hline
\end{tabular}


LAS AVES DEL DEPARTAMENTO DE LORETO

\begin{tabular}{|c|c|c|c|c|}
\hline Nombre científico & Nombre en inglés & Validación & Procedencia de Voucher & Amenaza/endemismo \\
\hline Leptopogon superciliaris von Tschudi, 1844 & Slaty-capped Flycatcher & VPR & CLO, LSUMZ, MUSM & \\
\hline Cnipodectes subbrunneus (Sclater, PL, 1860) & Brownish Twistwing & VPR & AMNH, CLO, LSUMZ, MUSM & \\
\hline Rhynchocyclus olivaceus (Temminck, 1820) & Olivaceous Flatbill & VPR & CLO, FMNH, LSUMZ, MUSM & \\
\hline Tolmomyias sulphurescens (von Spix, 1825) & Yellow-olive Flycatcher & VPR & FMNH, LSUMZ, MUSM & \\
\hline Tolmomyias traylori Schulenberg \& Parker, 1997 & Orange-eyed Flycatcher & VP & CLO, LSUMZ, MUSM & \\
\hline Tolmomyias assimilis (von Pelzeln, 1868) & Yellow-margined Flycatcher & VPR & CLO, LSUMZ, MCZ, MUSM & \\
\hline Tolmomyias poliocephalus (Taczanowski, 1884) & Gray-crowned Flycatcher & VPR & CLO, FMNH, LSUMZ, MCZ, MUSM & \\
\hline Tolmomyias flaviventris (zu Wied, 1831) & Yellow-breasted Flycatcher & VPR & CLO, CM, FMNH, LSUMZ, MCZ, MUSM, UF-FLMNH & \\
\hline Myiornis albiventris (von Berlepsch \& Stolzmann, 1894) & White-bellied Pygmy-Tyrant & $\mathrm{VP}$ & CLO, LSUMZ, MUSM & \\
\hline Myiornis ecaudatus (d'Orbigny \& Lafresnaye, 1837) & Short-tailed Pygmy-Tyrant & VPR & CLO, FMNH, LSUMZ, MUSM & \\
\hline Lophotriccus pileatus (von Tschudi, 1844) & Scale-crested Pygmy-Tyrant & VP & CLO & \\
\hline Lophotriccus vitiosus (Bangs \& Penard, 1921) & Double-banded Pygmy-Tyrant & VPR & CLO, LSUMZ, MCZ, MUSM, UF-FLMNH, UMMZ & \\
\hline Lophotriccus galeatus (Boddaert, 1783) & Helmeted Pygmy-Tyrant & $\mathrm{P}$ & & \\
\hline Hemitriccus minor (Snethlage, E, 1907) & Snethlage's Tody-Tyrant & $\mathrm{P}$ & & \\
\hline Hemitriccus flammulatus von Berlepsch, 1901 & Flammulated Pygmy-Tyrant & VPE & $\mathrm{MCZ}$ & \\
\hline Hemitriccus zosterops (von Pelzeln, 1868) & White-eyed Tody-Tyrant & VPR & CLO, FMNH, KU, LSUMZ, MUSM & \\
\hline Hemitriccus griseipectus (Snethlage, E, 1907) & White-bellied Tody-Tyrant & $P$ & & \\
\hline Hemitriccus iohannis (Snethlage, E, 1907) & Johannes's Tody-Tyrant & VPR & CLO, LSUMZ & \\
\hline Hemitriccus minimus (Todd, 1925) & Zimmer's Tody-Tyrant & $\mathrm{P}$ & & MIN-NT \\
\hline Hemitriccus rufigularis (Cabanis, 1873) & Buff-throated Tody-Tyrant & $V * \mathrm{P}$ & LSUMZ, MUSM & IUCN-NT, MIN-NT \\
\hline Poecilotriccus capitalis (Sclater, PL, 1857) & Black-and-white Tody-Flycatcher & VPR & CLO, LSUMZ & \\
\hline Poecilotriccus latirostris (von Pelzeln, 1868) & Rusty-fronted Tody-Flycatcher & VPR & CLO, FMNH, LSUMZ, NHMO & \\
\hline Poecilotriccus calopterus (Sclater, PL, 1857) & Golden-winged Tody-Flycatcher & VPR & CLO, LSUMZ & \\
\hline Todirostrum maculatum (Desmarest, 1806) & Spotted Tody-Flycatcher & $V * P R$ & CLO, CM, FMNH, LSUMZ, MCZ, MUSM, UF-FLMNH, USNM & \\
\hline Todirostrum cinereum (Linnaeus, 1766) & Common Tody-Flycatcher & $P$ & & \\
\hline Todirostrum chrysocrotaphum Strickland, 1850 & Yellow-browed Tody-Flycatcher & VPR & CLO, FMNH, LSUMZ, MUSM, USNM & \\
\hline Myiotriccus ornatus (Lafresnaye, 1853) & Ornate Flycatcher & $\mathrm{V} * \mathrm{PR}$ & CLO, MUSM & \\
\hline Hirundinea ferruginea (Gmelin, JF, 1788) & Cliff Flycatcher & VP & LSUMZ, MUSM & \\
\hline Pyrrhomyias cinnamomeus (d'Orbigny \& Lafresnaye, 1837) & Cinnamon Flycatcher & VP & LSUMZ & \\
\hline Zimmerius cinereicapilla (Cabanis, 1873) & Red-billed Tyrannulet & $P$ & & IUCN-VU \\
\hline Zimmerius villarejoi Alvarez \& Whitney, 2001 & Mishana Tyrannulet & $P$ & & IUCN-VU, MIN-VU, end \\
\hline Zimmerius gracilipes (Sclater \& Salvin, 1868) & Slender-footed Tyrannulet & VPR & CLO, FMNH, LSUMZ & \\
\hline Zimmerius viridiflavus (von Tschudi, 1844) & Peruvian Tyrannulet & $\mathrm{VP}$ & CLO & \\
\hline Stigmatura napensis Chapman, 1926 & Lesser Wagtail-Tyrant & VPR & CLO, LSUMZ, MUSM, UF-FLMNH & \\
\hline Ornithion inerme Hartlaub, 1853 & White-lored Tyrannulet & VPR & CLO, LSUMZ, MUSM & \\
\hline Camptostoma obsoletum (Temminck, 1824) & Southern Beardless-Tyrannulet & $\mathrm{V} * \mathrm{PR}$ & CLO, FMNH, LSUMZ, MUSM & \\
\hline Elaenia flavogaster (Thunberg, 1822) & Yellow-bellied Elaenia & VP & MUSM, ROM & \\
\hline Elaenia spectabilis von Pelzeln, 1868 & Large Elaenia & VPR & FMNH, LSUMZ, MUSM, NHMO & \\
\hline Elaenia albiceps (d'Orbigny \& Lafresnaye, 1837) & White-crested Elaenia & $\mathrm{VP}$ & LSUMZ, MCZ, MUSM & \\
\hline Elaenia parvirostris von Pelzeln, 1868 & Small-billed Elaenia & VPR & FMNH, LSUMZ, MUSM & \\
\hline
\end{tabular}


SALINAS ET AL.

\begin{tabular}{|c|c|c|c|c|}
\hline Nombre científico & Nombre en inglés & Validación & Procedencia de Voucher & Amenaza/endemismo \\
\hline Elaenia strepera Cabanis, 1883 & Slaty Elaenia & VPR & FMNH & \\
\hline Elaenia gigas Sclater, $\mathrm{PL}, 1871$ & Mottle-backed Elaenia & $\mathrm{VP}$ & LSUMZ & \\
\hline Elaenia pelzelni von Berlepsch, 1907 & Brownish Elaenia & $\mathrm{VP}$ & CLO, LSUMZ & \\
\hline Elaenia chiriquensis Lawrence, 1865 & Lesser Elaenia & $\mathrm{VP}$ & LSUMZ & \\
\hline Elaenia pallatangae Sclater, $\mathrm{PL}, 1862$ & Sierran Elaenia & $\mathrm{P}$ & & \\
\hline Tyrannulus elatus (Latham, 1790) & Tyrannulus elatus & $\mathrm{V*PR}$ & CLO, FMNH, LSUMZ, MCZ, MUSM & \\
\hline Myiopagis gaimardii (d'Orbigny, 1840) & Forest Elaenia & VPR & FMNH, LSUMZ, MUSM, UF-FLMNH & \\
\hline Myiopagis caniceps (Swainson, 1835) & Gray Elaenia & VPR & CLO, LSUMZ, MUSM & \\
\hline Myiopagis flavivertex (Sclater, PL, 1887) & Yellow-crowned Elaenia & VPR & AMNH, CLO, LSUMZ, MUSM, UF-FLMNH & \\
\hline Capsiempis flaveola (Lichtenstein, MHC, 1823) & Yellow Tyrannulet & VPR & LSUMZ & \\
\hline Phyllomyias burmeisteri Cabanis \& Heine, 1859 & Rough-legged Tyrannulet & $\mathrm{VP}$ & MUSM, LSUMZ & \\
\hline Phyllomyias griseiceps (Sclater \& Salvin, 1871) & Sooty-headed Tyrannulet & $P R$ & & \\
\hline Phyllomyias nigrocapillus (Lafresnaye, 1845) & Black-capped Tyrannulet & VPE & MCZ & \\
\hline Phaeomyias murina (von Spix, 1825) & Mouse-colored Tyrannulet & VPR & CLO, FMNH, LSUMZ, MUSM, NHMO & \\
\hline Mecocerculus minor (Taczanowski, 1879) & Sulphur-bellied Tyrannulet & $P$ & & \\
\hline Serpophaga cinerea (von Tschudi, 1844) & Torrent Tyrannulet & $P R$ & & \\
\hline Serpophaga hypoleuca Sclater \& Salvin, 1866 & River Tyrannulet & VPR & CLO, LSUMZ, MUSM & \\
\hline Attila cinnamomeus (Gmelin, JF, 1789) & Cinnamon Attila & VPR & CLO, FMNH, LSUMZ, MUSM & \\
\hline Attila citriniventris Sclater, $\mathrm{PL}, 1859$ & Citron-bellied Attila & VPR & CLO, KU, LSUMZ, MUSM & \\
\hline Attila bolivianus Lafresnaye, 1848 & Dull-capped Attila & VP & CLO, FMNH, LSUMZ, MUSM & \\
\hline Attila spadiceus (Gmelin, JF, 1789) & Bright-rumped Attila & VPR & CLO, FMNH, KU, LSUMZ, MUSM, UF-FLMNH & \\
\hline Legatus leucophaius (Vieillot, 1818) & Piratic Flycatcher & VPR & FMNH, LSUMZ, MUSM & \\
\hline Ramphotrigon megacephalum (Swainson, 1835) & Large-headed Flatbill & VP & MUSM & \\
\hline Ramphotrigon ruficauda (von Spix, 1825) & Rufous-tailed Flatbill & VPR & CLO, FMNH, LSUMZ, MCZ, MUSM, USNM & \\
\hline Ramphotrigon fuscicauda Chapman, 1925 & Dusky-tailed Flatbill & $\mathrm{VP}$ & MUSM & \\
\hline Pitangus sulphuratus (Linnaeus, 1766) & Great Kiskadee & V*PR & CLO, CM, FMNH, LSUMZ, MIZ, MUSM & \\
\hline Pitangus lictor (Lichtenstein, MHC, 1823) & Lesser Kiskadee & VPR & CLO, FMNH, LSUMZ, MUSM & \\
\hline Tyrannopsis sulphurea (von Spix, 1825) & Sulphury Flycatcher & VPR & FMNH, LSUMZ & \\
\hline Megarynchus pitangua (Linnaeus, 1766) & Boat-billed Flycatcher & VPR & CLO, FMNH, KU, LSUMZ, MCZ, MUSM, UF-FLMNH & \\
\hline Myiodynastes chrysocephalus (von Tschudi, 1844) & Golden-crowned Flycatcher & $P R$ & & \\
\hline Myiodynastes luteiventris Sclater, PL, 1859 & Sulphur-bellied Flycatcher & VP & FMNH & \\
\hline Myiodynastes maculatus (Statius Muller, 1776) & Streaked Flycatcher & V*PR & CLO, FMNH, LSUMZ, MUSM & \\
\hline Myiozetetes cayanensis (Linnaeus, 1766) & Rusty-margined Flycatcher & $\mathrm{PE}$ & & \\
\hline Myiozetetes similis (von Spix, 1825) & Social Flycatcher & VPR & CLO, CM, FMNH, KU, LSUMZ, MCZ, MUSM & \\
\hline Myiozetetes granadensis Lawrence, 1862 & Gray-capped Flycatcher & VPR & CLO, CM, FMNH, KU, LSUMZ, MUSM, NHMO & \\
\hline Myiozetetes luteiventris (Sclater, PL, 1858) & Dusky-chested Flycatcher & VPR & CLO, LSUMZ, MUSM & \\
\hline Conopias parvus (von Pelzeln, 1868) & Yellow-throated Flycatcher & VPR & CLO, LSUMZ, MUSM & \\
\hline Conopias trivirgatus (zu Wied, 1831) & Three-striped Flycatcher & VPR & LSUMZ & \\
\hline Conopias cinchoneti (von Tschudi, 1844) & Lemon-browed Flycatcher & $P E$ & & IUCN-VU \\
\hline Empidonomus varius (Vieillot, 1818) & Variegated Flycatcher & VPR & LSUMZ, MUSM & \\
\hline
\end{tabular}


LAS AVES DEL DEPARTAMENTO DE LORETO

\begin{tabular}{|c|c|c|c|c|}
\hline Nombre científico & Nombre en inglés & Validación & Procedencia de Voucher & Amenaza/endemismo \\
\hline $\begin{array}{l}\text { Empidonomus aurantioatrocristatus (d'Orbigny \& Lafresnaye, } \\
\text { 1837) }\end{array}$ & Crowned Slaty Flycatcher & VPR & FMNH, LSUMZ, MUSM & \\
\hline Tyrannus albogularis Burmeister, 1856 & White-throated Kingbird & VP & LSUMZ & \\
\hline Tyrannus melancholicus Vieillot, 1819 & Tropical Kingbird & $\mathrm{V} * \mathrm{PR}$ & $\mathrm{CLO}, \mathrm{CM}, \mathrm{FMNH}, \mathrm{KU}, \mathrm{LSUMZ}, \mathrm{MUSM}$ & \\
\hline Tyrannus savana Daudin, 1802 & Fork-tailed Flycatcher & $\mathrm{V} * \mathrm{PR}$ & LSUMZ, MUSM & \\
\hline Tyrannus tyrannus (Linnaeus, 1758) & Eastern Kingbird & $\mathrm{VP}$ & FMNH, MUSM & \\
\hline Rhytipterna simplex (Lichtenstein, MHC, 1823) & Grayish Mourner & $\mathrm{V} * \mathrm{PR}$ & CLO, FMNH, LSUMZ, MUSM, UF-FLMNH & \\
\hline Casiornis rufus (Vieillot, 1816) & Rufous Casiornis & PE & & \\
\hline Sirystes albocinereus Sclater \& Salvin, 1880 & White-rumped Sirystes & VPR & CLO, LSUMZ, MUSM, UF-FLMNH & \\
\hline Myiarchus tuberculifer (d'Orbigny \& Lafresnaye, 1837) & Dusky-capped Flycatcher & VPR & CLO, LSUMZ, MUSM & \\
\hline Myiarchus swainsoni Cabanis \& Heine, 1859 & Swainson's Flycatcher & VPR & LSUMZ, MCZ, MUSM & \\
\hline Myiarchus ferox (Gmelin, JF, 1789) & Short-crested Flycatcher & VPR & CLO, CM, FMNH, LSUMZ, MUSM, UF-FLMNH, YPM & \\
\hline Myiarchus cephalotes Taczanowski, 1880 & Pale-edged Flycatcher & VP & LSUMZ & \\
\hline Myiarchus crinitus (Linnaeus, 1758) & Great Crested Flycatcher & $p$ & & \\
\hline Myiarchus tyrannulus (Statius Muller, 1776) & Brown-crested Flycatcher & $\mathrm{V*P}$ & MUSM & \\
\hline Colonia colonus (Vieillot, 1818) & Long-tailed Tyrant & $\mathrm{V*P}$ & MUSM & \\
\hline Myiophobus roraimae (Salvin \& Godman, 1883) & Roraiman Flycatcher & $\mathrm{VP}$ & LSUMZ, MUSM & \\
\hline Myiophobus cryptoxanthus (Sclater, PL, 1861) & Olive-chested Flycatcher & PE & & \\
\hline Myiophobus fasciatus (Statius Muller, 1776) & Bran-colored Flycatcher & $\mathrm{VP}$ & LSUMZ, MCZ & \\
\hline Ochthoeca rufipectoralis (d'Orbigny \& Lafresnaye, 1837) & Rufous-breasted Chat-Tyrant & VPE & $\mathrm{MCZ}$ & \\
\hline Sublegatus obscurior Todd, 1920 & Amazonian Scrub-Flycatcher & VPR & $\mathrm{FMNH}$ & \\
\hline Sublegatus modestus (zu Wied, 1831) & Southern Scrub-Flycatcher & $\mathrm{VP}$ & MUSM & \\
\hline Pyrocephalus rubinus (Boddaert, 1783) & Vermilion Flycatcher & $V * P R$ & FMNH, MUSM, UMMZ & \\
\hline Fluvicola pica (Boddaert, 1783) & Pied Water-Tyrant & $P$ & & \\
\hline Fluvicola albiventer (von Spix, 1825) & Black-backed Water-Tyrant & $P R$ & & \\
\hline Arundinicola leucocephala (Linnaeus, 1764) & White-headed Marsh-Tyrant & $\mathrm{VP}$ & CLO, FMNH, LSUMZ & \\
\hline Knipolegus orenocensis von Berlepsch, 1884 & Riverside Tyrant & VPR & CLO, LSUMZ, MUSM, NHMO, UF-FLMNH & \\
\hline Knipolegus poecilurus (Sclater, $\mathrm{PL}, 1862$ ) & Rufous-tailed Tyrant & VP & CLO, LSUMZ, MUSM & \\
\hline Knipolegus poecilocercus (von Pelzeln, 1868) & Amazonian Black-Tyrant & $\mathrm{VP}$ & MUSM & \\
\hline Muscisaxicola fluviatilis Sclater \& Salvin, 1866 & Little Ground-Tyrant & $\mathrm{VP}$ & LSUMZ, MUSM & \\
\hline Muscisaxicola albilora Lafresnaye, 1855 & White-browed Ground-Tyrant & VPE & $\mathrm{MCZ}$ & \\
\hline Ochthornis littoralis (von Pelzeln, 1868) & Drab Water Tyrant & VPR & CLO, CM, FMNH, LSUMZ, MCZ, MUSM, ROM & \\
\hline Cnemotriccus fuscatus (zu Wied, 1831) & Fuscous Flycatcher & VPR & CLO, LSUMZ, MCZ, MUSM, NHMO & \\
\hline Lathrotriccus euleri (Cabanis, 1868) & Euler's Flycatcher & $V * P R$ & CLO, FMNH, LSUMZ, MUSM, NHMO, RMNH & \\
\hline Mitrephanes olivaceus von Berlepsch \& Stolzmann, 1894 & Olive Flycatcher & $\mathrm{v}$ & MUSM & \\
\hline Sayornis nigricans (Swainson, 1827) & Black Phoebe & VP & LSUMZ, MUSM & \\
\hline Empidonax traillii (Audubon, 1828) & Willow Flycatcher & PE & & \\
\hline Empidonax alnorum Brewster, 1895 & Alder Flycatcher & VPR & FMNH & \\
\hline Contopus cooperi (Nuttall, 1832) & Olive-sided Flycatcher & $P R$ & & IUCN-NT \\
\hline Contopus fumigatus (d'Orbigny \& Lafresnaye, 1837) & Smoke-colored Pewee & $P R$ & & \\
\hline Contopus sordidulus Sclater, PL, 1859 & Western Wood-Pewee & $P$ & & \\
\hline
\end{tabular}


SALINAS ET AL.

\begin{tabular}{|c|c|c|c|c|}
\hline Nombre científico & Nombre en inglés & Validación & Procedencia de Voucher & Amenaza/endemismo \\
\hline Contopus virens (Linnaeus, 1766) & Eastern Wood-Pewee & VPR & CLO, MUSM, UF-FLMNH, YPM & \\
\hline Contopus nigrescens (Sclater \& Salvin, 1880) & Blackish Pewee & $\mathrm{VP}$ & CLO, LSUMZ & \\
\hline \multicolumn{5}{|l|}{ Orden Passeriformes: Familia Vireonidae } \\
\hline Cyclarhis gujanensis (Gmelin, JF, 1789) & Rufous-browed Peppershrike & $\mathrm{VP}$ & LSUMZ, MUSM & \\
\hline Hylophilus semicinereus Sclater \& Salvin, 1867 & Gray-chested Greenlet & $P$ & & \\
\hline Hylophilus thoracicus Temminck, 1822 & Lemon-chested Greenlet & VPR & CLO, LSUMZ & \\
\hline Vireolanius leucotis (Swainson, 1838) & Slaty-capped Shrike-Vireo & VPR & CLO, LSUMZ, MUSM & \\
\hline Tunchiornis ochraceiceps Sclater, PL, 1860 & Tawny-crowned Greenlet & VPR & CLO, KU, LSUMZ, MUSM & \\
\hline Pachysy/via hypoxantha von Pelzeln, 1868 & Dusky-capped Greenlet & VPR & CLO, LSUMZ, MUSM & \\
\hline Vireo leucophrys (Lafresnaye, 1844) & Brown-capped Vireo & $\mathrm{VP}$ & LSUMZ & \\
\hline Vireo olivaceus (Linnaeus, 1766) & Red-eyed Vireo & $\mathrm{V}$ PR & CLO, FMNH, LSUMZ, MUSM, NHMO, ROM & \\
\hline Vireo chivi (Vieillot, 1817) & Chivi Vireo & VPR & MUSM, FMNH & \\
\hline Vireo flavoviridis (Cassin, 1851) & Yellow-green Vireo & $\mathrm{VP}$ & FMNH & \\
\hline Vireo altiloquus (Vieillot, 1808) & Black-whiskered Vireo & $\mathrm{PE}$ & & \\
\hline \multicolumn{5}{|l|}{ Orden Passeriformes: Familia Corvidae } \\
\hline Cyanocorax violaceus Du Bus, 1847 & Violaceous Jay & V*PR & CLO, FMNH, LSUMZ, UMMZ & \\
\hline Cyanocorax yncas (Boddaert, 1783) & Green Jay & $\mathrm{VP}$ & LSUMZ, MUSM, UF-FLMNH & \\
\hline \multicolumn{5}{|l|}{ Orden Passeriformes: Familia Hirundinidae } \\
\hline Pygochelidon cyanoleuca (Vieillot, 1817) & Blue-and-white Swallow & V*PR & FMNH, LSUMZ, MUSM & \\
\hline Pygochelidon melanoleuca (zu Wied-Neuwied, 1820) & Black-collared Swallow & $\mathrm{PE}$ & & \\
\hline Atticora fasciata (Gmelin, JF, 1789) & White-banded Swallow & $\mathrm{V} * \mathrm{PR}$ & $\mathrm{CLO}, \mathrm{FMNH}, \mathrm{KU}, \mathrm{LSUMZ}, \mathrm{MUSM}$ & \\
\hline Atticora tibialis (Cassin, 1853) & White-thighed Swallow & $P R$ & & \\
\hline Stelgidopteryx ruficollis (Vieillot, 1817) & Southern Rough-winged Swallow & V*PR & CLO, CM, FMNH, LSUMZ, MCZ, MUSM, YPM & \\
\hline Progne tapera (Linnaeus, 1766) & Brown-chested Martin & $\mathrm{V}$ PR & CLO, FMNH, LSUMZ, MCZ, MUSM & \\
\hline Progne subis (Linnaeus, 1758) & Purple Martin & $P$ & & \\
\hline Progne chalybea (Gmelin, JF, 1789) & Gray-breasted Martin & $\mathrm{V*PR}$ & FMNH, KU, LSUMZ, MUSM & \\
\hline Progne elegans Baird, SF, 1865 & Southern Martin & $\mathrm{VP}$ & CLO & \\
\hline Tachycineta albiventer (Boddaert, 1783) & White-winged Swallow & V*PR & FMNH, KU, LSUMZ, MCZ, MUSM, UMMZ & \\
\hline Riparia riparia (Linnaeus, 1758) & Bank Swallow & VPR & $\mathrm{CLO}$ & \\
\hline Hirundo rustica Linnaeus, 1758 & Barn Swallow & VPR & MUSM & \\
\hline Petrochelidon pyrrhonota (Vieillot, 1817) & Cliff Swallow & P & & \\
\hline \multicolumn{5}{|l|}{ Orden Passeriformes: Familia Troglodytidae } \\
\hline Microcerculus marginatus (Sclater, PL, 1855) & Scaly-breasted Wren & VPR & CLO, FMNH, KU, LSUMZ, MUSM, NHMO & \\
\hline Microcerculus bambla (Boddaert, 1783) & Wing-banded Wren & $P R$ & & \\
\hline Odontorchilus branickii (Taczanowski \& von Berlepsch, 1885) & Gray-mantled Wren & $\mathrm{P}$ & & \\
\hline Troglodytes aedon Vieillot, 1809 & House Wren & VPR & FMNH, LSUMZ & \\
\hline Troglodytes solstitialis Sclater, PL, 1859 & Mountain Wren & VR & LSUMZ & \\
\hline Campylorhynchus turdinus (zu Wied-Neuwied, 1821) & Thrush-like Wren & VPR & CLO, FMNH, KU, LSUMZ, MUSM, UF-FLMNH & \\
\hline Pheugopedius genibarbis (Swainson, 1838) & Moustached Wren & VP & CLO, FMNH & \\
\hline Pheugopedius coraya (Gmelin, JF, 1789) & Coraya Wren & VPR & CLO, CM, LSUMZ, MUSM, UF-FLMNH & \\
\hline
\end{tabular}


LAS AVES DEL DEPARTAMENTO DE LORETO

\begin{tabular}{|c|c|c|c|c|}
\hline Nombre científico & Nombre en inglés & Validación & Procedencia de Voucher & Amenaza/endemismo \\
\hline Pheugopedius sclateri (Taczanowski, 1879) & Speckle-breasted Wren & $\mathrm{v}$ & MUSM & \\
\hline Cantorchilus leucotis (Lafresnaye, 1845) & Buff-breasted Wren & VPR & CLO, CM, FMNH, LSUMZ, MUSM, UF-FLMNH & \\
\hline Cantorchilus griseus (Todd, 1925) & Gray Wren & $\mathrm{PE}$ & & \\
\hline Henicorhina leucosticta (Cabanis, 1847) & White-breasted Wood-Wren & VPR & KU, LSUMZ, MUSM & \\
\hline Henicorhina leucoptera Fitzpatrick, Terborgh \& Willard, 1977 & Bar-winged Wood-Wren & VP & LSUMZ, MUSM & IUCN-NT, MIN-NT \\
\hline Henicorhina leucophrys (von Tschudi, 1844) & Gray-breasted Wood-Wren & VPR & LSUMZ, MUSM & \\
\hline Cyphorhinus arada (Hermann, 1783) & Musician Wren & VPR & CLO, CM, FMNH, LSUMZ, MCZ, MUSM, UF-FLMNH & \\
\hline \multicolumn{5}{|l|}{ Orden Passeriformes: Familia Polioptilidae } \\
\hline Microbates collaris (von Pelzeln, 1868) & Collared Gnatwren & $\mathrm{VP}$ & CLO, LSUMZ & \\
\hline Microbates cinereiventris (Sclater, PL, 1855) & Tawny-faced Gnatwren & VP & AMNH, CLO, KU, LSUMZ, UF-FLMNH & \\
\hline Ramphocaenus melanurus Vieillot, 1819 & Trilling Gnatwren & $V * P R$ & BMNH, CLO, LSUMZ, MUSM & \\
\hline Ramphocaenus sticturus Hellmayr, 1902 & Chattering Gnatwren & VR & LSUMZ & \\
\hline Polioptila plumbea (Gmelin, JF, 1788) & Tropical Gnatcatcher & $\mathrm{V} * \mathrm{PR}$ & LSUMZ, MUSM & \\
\hline Polioptila clementsi Whitney \& Alvarez Alonso, 2005 & Iquitos Gnatcatcher & $\mathrm{VP}$ & CLO, LSUMZ, MUSM & $\mathrm{MIN}-\mathrm{CR}$, end \\
\hline \multicolumn{5}{|l|}{ Orden Passeriformes: Familia Donacobiidae } \\
\hline Donacobius atricapilla (Linnaeus, 1766) & Black-capped Donacobius & $V * P R$ & CLO, CM, FMNH, LSUMZ, MUSM, UMMZ & \\
\hline \multicolumn{5}{|l|}{ Orden Passeriformes: Familia Cinclidae } \\
\hline Cinclus leucocephalus von Tschudi, 1844 & White-capped Dipper & $\mathrm{VP}$ & AMNH & \\
\hline \multicolumn{5}{|l|}{ Orden Passeriformes: Familia Turdidae } \\
\hline Myadestes ralloides (d'Orbigny, 1840) & Andean Solitaire & $\mathrm{VP}$ & LSUMZ & \\
\hline Catharus dryas (Gould, 1855) & Spotted Nightingale-Thrush & $\mathrm{VP}$ & CLO, LSUMZ, MUSM & \\
\hline Catharus fuscescens (Stephens, 1817) & Veery & PR & & \\
\hline Catharus minimus (Lafresnaye, 1848) & Gray-cheeked Thrush & VPR & MUSM & \\
\hline Catharus ustulatus (Nuttall, 1840) & Swainson's Thrush & VPR & FMNH, MUSM, YPM & \\
\hline Entomodestes leucotis (von Tschudi, 1844) & White-eared Solitaire & VP & CLO & \\
\hline Turdus leucops Taczanowski, 1877 & Pale-eyed Thrush & VP & LSUMZ, MUSM & \\
\hline Turdus hauxwelli Lawrence, 1869 & Hauxwell's Thrush & VPR & AMNH, CLO, CM, FMNH, LSUMZ, MUSM, YPM & \\
\hline Turdus sanchezorum O'Neill, Lane, \& Naka, 2011 & Varzea Thrush & VP & CLO & \\
\hline Turdus lawrencii Coues, 1880 & Lawrence's Thrush & VPR & CLO, FMNH, LSUMZ, MUSM, UF-FLMNH & \\
\hline Turdus ignobilis Sclater, PL, 1858 & Black-billed Thrush & VPR & CLO, CM, FMNH, KU, LSUMZ, MUSM, YPM & \\
\hline Turdus nigriceps Cabanis, 1874 & Andean Slaty Thrush & VPR & LSUMZ & \\
\hline Turdus serranus von Tschudi, 1844 & Glossy-black Thrush & VPR & LSUMZ, MUSM & \\
\hline Turdus albicollis Royle, 1840 & White-necked Thrush & VPR & CLO, FMNH, KU, LSUMZ, MUSM, UF-FLMNH & \\
\hline \multicolumn{5}{|l|}{ Orden Passeriformes: Familia Mimidae } \\
\hline Mimus gilvus (Vieillot, 1808) & Tropical Mockingbird & $P$ & & \\
\hline \multicolumn{5}{|l|}{ Orden Passeriformes: Familia Passeridae } \\
\hline Passer domesticus (Linnaeus, 1758) & House Sparrow & $\mathrm{P}$ & & \\
\hline \multicolumn{5}{|l|}{ Orden Passeriformes: Familia Fringillidae } \\
\hline Chlorophonia cyanocephala (Vieillot, 1819) & Golden-rumped Euphonia & $\mathrm{PE}$ & & \\
\hline Chlorophonia cyanea (Thunberg, 1822) & Blue-naped Chlorophonia & $V * P$ & CLO, LSUMZ, MUSM, UF-FLMNH & \\
\hline
\end{tabular}


SALINAS ET AL.

\begin{tabular}{|c|c|c|c|c|}
\hline Nombre científico & Nombre en inglés & Validación & Procedencia de Voucher & Amenaza/endemismo \\
\hline Euphonia plumbea Du Bus, 1855 & Plumbeous Euphonia & $P R$ & & \\
\hline Euphonia chlorotica (Linnaeus, 1766) & Purple-throated Euphonia & VPR & UF-FLMNH & \\
\hline Euphonia chrysopasta Sclater \& Salvin, 1869 & Golden-bellied Euphonia & V*PR & CLO, FMNH, LSUMZ, MCZ, MUSM & \\
\hline Euphonia minuta Cabanis, 1849 & White-vented Euphonia & $V * P R$ & CLO, FMNH, LSUMZ, MUSM, YPM & \\
\hline Euphonia laniirostris d'Orbigny \& Lafresnaye, 1837 & Thick-billed Euphonia & V*PR & CLO, FMNH, LSUMZ, MUSM & \\
\hline Euphonia xanthogaster Sundevall, 1834 & Orange-bellied Euphonia & V*PR & AMNH, CLO, CM, FMNH, LSUMZ, MUSM, ROM & \\
\hline Euphonia mesochrysa Salvadori, 1873 & Bronze-green Euphonia & $\mathrm{VP}$ & CLO, LSUMZ, MUSM & \\
\hline Euphonia rufiventris (Vieillot, 1819) & Rufous-bellied Euphonia & V*PR & CLO, FMNH, LSUMZ, MCZ, MUSM, USNM & \\
\hline \multicolumn{5}{|l|}{ Orden Passeriformes: Familia Passerellidae } \\
\hline Chlorospingus flavigularis (Sclater, PL, 1852) & Yellow-throated Chlorospingus & VP & LSUMZ & \\
\hline Chlorospingus canigularis (Lafresnaye, 1848) & Ashy-throated Chlorospingus & PE & & \\
\hline Chlorospingus flavopectus (Lafresnaye, 1840) & Common Chlorospingus & VPR & LSUMZ & \\
\hline Ammodramus aurifrons (von Spix, 1825) & Yellow-browed Sparrow & VPR & CM, CLO, FMNH, KU, LSUMZ, MCZ, MUSM, NHMO, UF-FLMNH & \\
\hline Arremon aurantiirostris Lafresnaye, 1847 & Orange-billed Sparrow & $\mathrm{VP}$ & LSUMZ, ROM & \\
\hline Arremon taciturnus (Hermann, 1783) & Pectoral Sparrow & VP & LSUMZ & \\
\hline Arremon brunneinucha (Lafresnaye, 1839) & Chestnut-capped Brushfinch & VP & FMNH, LSUMZ, MUSM & \\
\hline Zonotrichia capensis (Statius Muller, 1776) & Rufous-collared Sparrow & $P R$ & & \\
\hline Atlapetes tricolor (Taczanowski, 1875) & Tricolored Brushfinch & VP & LSUMZ, MUSM & \\
\hline Atlapetes latinuchus (Du Bus, 1855) & Yellow-breasted Brushfinch & $\mathrm{V}$ & LSUMZ & \\
\hline \multicolumn{5}{|l|}{ Orden Passeriformes: Familia Icteridae } \\
\hline Dolichonyx oryzivorus (Linnaeus, 1758) & Bobolink & VPR & MUSM & CMS-II \\
\hline Leistes militaris (Linnaeus, 1758) & Red-breasted Meadowlark & VPR & LSUMZ, MUSM, YPM & \\
\hline Amblycercus holosericeus (Deppe, 1830) & Yellow-billed Cacique & $P E$ & & \\
\hline Psarocolius angustifrons (von Spix, 1824) & Russet-backed Oropendola & V*PR & CLO, CM, FMNH, KU, LSUMZ, MCZ, MUSM, MVZ, UF-FLMNH & \\
\hline Psarocolius viridis (Statius Muller, 1776) & Green Oropendola & $V * P R$ & CLO, LSUMZ, MCZ, MUSM, RMNH & \\
\hline Psarocolius decumanus (Pallas, 1769) & Crested Oropendola & VPR & CLO, FMNH, LSUMZ, MCZ, MUSM, UF-FLMNH & \\
\hline Psarocolius bifasciatus (von Spix, 1824) & Olive Oropendola & $V * P R$ & CLO, FMNH, LSUMZ, MUSM & \\
\hline Cacicus solitarius (Vieillot, 1816) & Solitary Black Cacique & $V * P R$ & CLO, FMNH, MUSM, MVZ, UF-FLMNH & \\
\hline Cacicus sclateri (Dubois, AJC, 1887) & Ecuadorian Cacique & VPR & CLO, FMNH, LSUMZ, MUSM & \\
\hline Cacicus uropygialis (Lafresnaye, 1843) & Scarlet-rumped Cacique & VP & UF-FLMNH & \\
\hline Cacicus cela (Linnaeus, 1758) & Yellow-rumped Cacique & $V * P R$ & CLO, CM, FMNH, LSUMZ, MUSM, UMMZ, YPM & \\
\hline Cacicus latirostris (Swainson, 1838) & Band-tailed Cacique & VPR & CLO, CM, FMNH, LSUMZ, MUSM, ROM, UF-FLMNH, YPM & \\
\hline Cacicus haemorrhous (Linnaeus, 1766) & Red-rumped Cacique & VPR & LSUMZ & \\
\hline Cacicus oseryi (Deville, 1849) & Casqued Cacique & VPR & CLO, FMNH, LSUMZ, MUSM, YPM & \\
\hline Icterus croconotus (Wagler, 1829) & Orange-backed Troupial & $\mathrm{V} * \mathrm{PR}$ & CLO, FMNH, LSUMZ, MUSM, YPM & \\
\hline Icterus graceannae Cassin, 1867 & White-edged Oriole & $\mathrm{Vt}$ & MUSM & \\
\hline Icterus cayanensis (Linnaeus, 1766) & Epaulet Oriole & VPR & CLO, FMNH, LSUMZ, MUSM, UMMZ & \\
\hline Icterus chrysocephalus (Linnaeus, 1766) & Moriche Oriole & VR & LSUMZ, MUSM & \\
\hline Molothrus oryzivorus (Gmelin, JF, 1788) & Giant Cowbird & $V * P R$ & FMNH, MUSM, UF-FLMNH & \\
\hline Molothrus bonariensis (Gmelin, JF, 1789) & Shiny Cowbird & VPR & CLO, FMNH, LSUMZ, MUSM & \\
\hline
\end{tabular}


LAS AVES DEL DEPARTAMENTO DE LORETO

\begin{tabular}{|c|c|c|c|c|}
\hline Nombre científico & Nombre en inglés & Validación & Procedencia de Voucher & Amenaza/endemismo \\
\hline Lampropsar tanagrinus (von Spix, 1824) & Velvet-fronted Grackle & VPR & CLO, FMNH, LSUMZ, MUSM, RMNH, UF-FLMNH & \\
\hline Gymnomystax mexicanus (Linnaeus, 1766) & Oriole Blackbird & $V * P R$ & CLO, CM, LSUMZ, MNHN, MUSM, NHMO & \\
\hline Agelasticus xanthophthalmus (Short, 1969) & Pale-eyed Blackbird & PR & & \\
\hline Chrysomus icterocephalus (Linnaeus, 1766) & Yellow-hooded Blackbird & VPR & CLO, FMNH, LSUMZ, MUSM, YPM & \\
\hline \multicolumn{5}{|l|}{ Orden Passeriformes: Familia Parulidae } \\
\hline Parkesia noveboracensis (Gmelin, JF, 1789) & Northern Waterthrush & $P$ & & \\
\hline Mniotilta varia (Linnaeus, 1766) & Black-and-white Warbler & VP & MUSM & \\
\hline Oporornis agilis (Wilson, A, 1812) & Connecticut Warbler & $\mathrm{P}$ & & \\
\hline Setophaga ruticilla (Linnaeus, 1758) & American Redstart & $P R$ & & \\
\hline Setophaga cerulea (Wilson, A, 1810) & Cerulean Warbler & P & & IUCN-NT, CMS-I \\
\hline Setophaga pitiayumi (Vieillot, 1817) & Tropical Parula & $P$ & & \\
\hline Setophaga fusca (Statius Muller, 1776) & Blackburnian Warbler & $\mathrm{E}$ & & \\
\hline Setophaga petechia (Linnaeus, 1766) & Yellow Warbler & VP & CLO & \\
\hline Setophaga striata (Forster, JR, 1772) & Blackpoll Warbler & VPR & ROM & IUCN-NT \\
\hline Myiothlypis fulvicauda (von Spix, 1825) & Buff-rumped Warbler & VPR & CLO, FMNH, KU, LSUMZ, MUSM & \\
\hline Myiothlypis chrysogaster (von Tschudi, 1844) & Golden-bellied Warbler & VP & CLO, LSUMZ, MUSM & \\
\hline Basileuterus tristriatus (von Tschudi, 1844) & Three-striped Warbler & VP & CLO, LSUMZ, MUSM & \\
\hline Cardellina canadensis (Linnaeus, 1766) & Canada Warbler & $P$ & & \\
\hline Myioborus miniatus (Swainson, 1827) & Slate-throated Redstart & VPR & CLO, LSUMZ, MUSM & \\
\hline \multicolumn{5}{|l|}{ Orden Passeriformes: Familia Mitrospingidae } \\
\hline Lamprospiza melanoleuca (Vieillot, 1817) & Red-billed Pied Tanager & $\mathrm{VP}$ & CLO, LSUMZ, MUSM & \\
\hline \multicolumn{5}{|l|}{ Orden Passeriformes: Familia Cardinalidae } \\
\hline Piranga flava (Vieillot, 1822) & Hepatic Tanager & $\mathrm{VP}$ & LSUMZ, MUSM & \\
\hline Piranga rubra (Linnaeus, 1758) & Summer Tanager & PR & & \\
\hline Piranga olivacea (Gmelin, JF, 1789) & Scarlet Tanager & VPR & $\mathrm{CM}, \mathrm{FMNH}$ & \\
\hline Piranga leucoptera (Trudeau, 1840) & White-winged Tanager & $\mathrm{VP}$ & CLO, LSUMZ, MUSM & \\
\hline Habia rubica (Vieillot, 1817) & Red-crowned Ant-Tanager & $\mathrm{V} * \mathrm{PR}$ & CLO, LSUMZ, MIZ, MUSM, UF-FLMNH, YPM & \\
\hline Chlorothraupis carmioli (Lawrence, 1868) & Carmiol's Tanager & VP & CLO, LSUMZ, MUSM & \\
\hline Cyanoloxia rothschildii (Bartlett, E, 1890) & Amazonian Grosbeak & VPR & CLO, FMNH, KU, LSUMZ, MUSM, UF-FLMNH & \\
\hline \multicolumn{5}{|l|}{ Orden Passeriformes: Familia Thraupidae } \\
\hline Wetmorethraupis sterrhopteron Lowery \& O'Neill, 1964 & Orange-throated Tanager & $P R$ & & IUCN-VU, MIN-VU \\
\hline Volatinia jacarina (Linnaeus, 1766) & Blue-black Grassquit & VPR & LSUMZ, MUSM, NHMO & \\
\hline Thraupis palmarum (zu Wied-Neuwied, 1821) & Palm Tanager & VPR & CLO, CM, FMNH, LSUMZ, MCZ, MUSM, YPM & \\
\hline Thraupis episcopus (Linnaeus, 1766) & Blue-gray Tanager & $\mathrm{V} * \mathrm{PR}$ & CLO, CM, CUMV, FMNH, KU, LSUMZ, MCZ, MUSM, NHMO, UMMZ, YPM & \\
\hline Thlypopsis sordida (d'Orbigny \& Lafresnaye, 1837) & Orange-headed Tanager & VPR & CLO, LSUMZ, MUSM, NHMO & \\
\hline Tersina viridis (Illiger, 1811) & Swallow Tanager & V*PR & FMNH, LSUMZ, MCZ, MUSM, ROM, USNM & \\
\hline Tangara xanthocephala (von Tschudi, 1844) & Saffron-crowned Tanager & VPR & LSUMZ & \\
\hline Tangara velia (Linnaeus, 1758) & Opal-rumped Tanager & VPR & LSUMZ, MUSM, WFVZ & \\
\hline Tangara vassorii (Boissonneau, 1840) & Blue-and-black Tanager & $\mathrm{V}$ & LSUMZ & \\
\hline Tangara nigroviridis (Lafresnaye, 1843) & Beryl-spangled Tanager & $P$ & & \\
\hline
\end{tabular}


SALINAS ET AL.

\begin{tabular}{|c|c|c|c|c|}
\hline Nombre científico & Nombre en inglés & Validación & Procedencia de Voucher & Amenaza/endemismo \\
\hline Tangara schrankii (von Spix, 1825) & Green-and-gold Tanager & $\mathrm{V} * \mathrm{PR}$ & CLO, CM, FMNH, LSUMZ, MNHN, MUSM, ROM, UF-FLMNH, USNM, YPM & \\
\hline Tangara parzudakii (Lafresnaye, 1843) & Flame-faced Tanager & $\mathrm{VP}$ & LSUMZ, MUSM & \\
\hline Tangara mexicana (Linnaeus, 1766) & Turquoise Tanager & $\mathrm{V} * \mathrm{PR}$ & FMNH, LSUMZ, MUSM, USNM, YPM & \\
\hline Tangara gyrola (Linnaeus, 1758) & Bay-headed Tanager & VPR & AMNH, CLO, LSUMZ, MUSM & \\
\hline Tangara cyanotis (Sclater, PL, 1858) & Blue-browed Tanager & $\mathrm{VP}$ & LSUMZ & \\
\hline Tangara chrysotis (Du Bus, 1846) & Golden-eared Tanager & $\mathrm{V}$ & LSUMZ & \\
\hline Tangara chilensis (Vigors, 1832) & Paradise Tanager & $\mathrm{V} * \mathrm{PR}$ & CLO, FMNH, LSUMZ, MCZ, MNHN, MUSM, ROM, UF-FLMNH, YPM & \\
\hline Tangara callophrys (Cabanis, 1849) & Opal-crowned Tanager & $\mathrm{V}$ PR & FMNH, LSUMZ, MCZ, MUSM, YPM & \\
\hline Tangara arthus Lesson, 1832 & Golden Tanager & VPR & LSUMZ & \\
\hline Tachyphonus surinamus (Linnaeus, 1766) & Fulvous-crested Tanager & VPR & FMNH, KU, LSUMZ, MUSM, YPM & \\
\hline Tachyphonus rufus (Boddaert, 1783) & White-lined Tanager & $P$ & & \\
\hline Tachyphonus phoenicius Swainson, 1838 & Red-shouldered Tanager & VPR & LSUMZ, MUSM & \\
\hline Stilpnia viridicollis (Taczanowski, 1884) & Silvery Tanager & VR & LSUMZ & \\
\hline Stilpnia nigrocincta (Bonaparte, 1838) & Masked Tanager & VPR & LSUMZ, MUSM, ROM & \\
\hline Stilpnia cyanicollis (d'Orbigny \& Lafresnaye, 1837) & Blue-necked Tanager & VPR & LSUMZ & \\
\hline Sporophila schistacea (Lawrence, 1862) & Slate-colored Seedeater & $\mathrm{P}$ & & \\
\hline Sporophila nigricollis (Vieillot, 1823) & Yellow-bellied Seedeater & $\mathrm{VP}$ & $\mathrm{CM}, \mathrm{FMNH}, \mathrm{MCZ}$ & \\
\hline Sporophila murallae Chapman, 1915 & Caquetá Seedeater & VPR & CLO, CM, FMNH, LSUMZ, MUSM, NHMO, YPM & \\
\hline Sporophila luctuosa (Lafresnaye, 1843) & Black-and-white Seedeater & $\mathrm{V}$ PR & FMNH, MUSM, MVZ & \\
\hline Sporophila lineola (Linnaeus, 1758) & Lined Seedeater & VPR & CM, FMNH, MUSM, MVZ, YPM & \\
\hline Sporophila crassirostris (Gmelin, JF, 1789) & Large-billed Seed-Finch & $\mathrm{V} * \mathrm{PR}$ & $\mathrm{CM}, \mathrm{FMNH}, \mathrm{MUSM}$ & \\
\hline Sporophila castaneiventris Cabanis, 1849 & Chestnut-bellied Seedeater & $\mathrm{V}$ PR & CLO, CM, FMNH, KU, LSUMZ, MCZ, MUSM, NHMO, UMMZ, USNM, WFVZ & \\
\hline Sporophila caerulescens (Vieillot, 1823) & Double-collared Seedeater & VPR & MUSM & \\
\hline Sporophila bouvronides (Lesson, 1831) & Lesson's Seedeater & VPR & CM, LSUMZ, MUSM & \\
\hline Sporophila atrirostris (Sclater \& Salvin, 1878) & Black-billed Seed-Finch & VPR & $\mathrm{CM}$ & \\
\hline Sporophila angolensis (Linnaeus, 1766) & Chestnut-bellied Seed-Finch & $\mathrm{V} * \mathrm{PR}$ & CLO, CM, FMNH, LSUMZ, MIZ, MUSM, NHMO, TCWC, UF-FLMNH & \\
\hline Sicalis flaveola (Linnaeus, 1766) & Saphron Finch & $\mathrm{PR}$ & & \\
\hline Schistochlamys melanopis (Latham, 1790) & Black-faced Tanager & VPR & LSUMZ, MUSM & \\
\hline Saltator maximus (Statius Muller, 1776) & Buff-throated Saltator & $\mathrm{V} * \mathrm{PR}$ & CLO, CM, FMNH, LSUMZ, MUSM, UF-FLMNH, UMMZ, USNM, YPM & \\
\hline Saltator coerulescens Vieillot, 1817 & Grayish Saltator & $\mathrm{V} * \mathrm{PR}$ & CLO, CM, FMNH, LSUMZ, MCZ, MUSM, UF-FLMNH, USNM, YPM & \\
\hline Saltator grossus (Linnaeus, 1766) & Slate-colored Grosbeak & $\mathrm{V} * \mathrm{PR}$ & CLO, FMNH, KU, LSUMZ, MUSM, UMMZ & \\
\hline Ramphocelus nigrogularis (von Spix, 1825) & Masked Crimson Tanager & $\mathrm{V} * \mathrm{PR}$ & CLO, CM, FMNH, LSUMZ, MCZ, MUSM, ROM, WFVZ, YPM & \\
\hline Ramphocelus melanogaster (Swainson, 1838) & Black-bellied Tanager & VPR & CLO & end \\
\hline Ramphocelus carbo (Pallas, 1764) & Silver-beaked Tanager & $\mathrm{V} * \mathrm{PR}$ & CLO, CM, FMNH, LSUMZ, MUSM, MVZ, NHMO, TCWC, USNM, YPM & \\
\hline Paroaria gularis (Linnaeus, 1766) & Red-capped Cardinal & $\mathrm{V} * \mathrm{PR}$ & CLO, CM, FMNH, LSUMZ, MCZ, MUSM, WFVZ & \\
\hline Parkerthraustes humeralis (Lawrence, 1867) & Yellow-shouldered Grosbeak & $\mathrm{VP}$ & CLO, LSUMZ & \\
\hline Nemosia pileata (Boddaert, 1783) & Hooded Tanager & $\mathrm{VP}$ & CLO, FMNH, LSUMZ, MUSM & \\
\hline Loriotus rufiventer (von Spix, 1825) & Yellow-crested Tanager & VPR & CLO, LSUMZ, MUSM & \\
\hline Loriotus luctuosus d'Orbigny \& Lafresnaye, 1837 & White-shouldered Tanager & VPR & LSUMZ, MUSM & \\
\hline Loriotus cristatus (Linnaeus, 1766) & Flame-crested Tanager & $\mathrm{V} * \mathrm{PR}$ & AMNH, FMNH, KU, LSUMZ, MVZ, ROM, UMMZ & \\
\hline
\end{tabular}


LAS AVES DEL DEPARTAMENTO DE LORETO

\begin{tabular}{|c|c|c|c|c|}
\hline Nombre científico & Nombre en inglés & Validación & Procedencia de Voucher & Amenaza/endemismo \\
\hline Lanio versicolor (d'Orbigny \& Lafresnaye, 1837) & White-winged Shrike-Tanager & $\mathrm{VP}$ & LSUMZ, MUSM & \\
\hline Lanio fulvus (Boddaert, 1783) & Fulvous Shrike-Tanager & VPR & CLO, LSUMZ, UF-FLMNH & \\
\hline Ixothraupis xanthogastra (Sclater, PL, 1851) & Yellow-bellied Tanager & $\mathrm{V} * \mathrm{PR}$ & LSUMZ, MUSM, ROM & \\
\hline Ixothraupis varia (Statius Muller, 1776) & Dotted Tanager & VPR & CLO, LSUMZ, MUSM & \\
\hline Ixothraupis punctata (Linnaeus, 1766) & Spotted Tanager & $P R$ & & \\
\hline Iridosornis rufivertex (Lafresnaye, 1842) & Golden-crowned Tanager & $\mathrm{v}$ & LSUMZ, MUSM & \\
\hline Iridosornis analis (von Tschudi, 1844) & Yellow-throated Tanager & VP & LSUMZ, MUSM & \\
\hline Iridophanes pulcherrimus (Sclater, PL, 1853) & Golden-collared Honeycreeper & VPE & LSUMZ & \\
\hline Hemithraupis guira (Linnaeus, 1766) & Guira Tanager & $\mathrm{VP}$ & CLO, LSUMZ, MUSM & \\
\hline Hemithraupis flavicollis (Vieillot, 1818) & Yellow-backed Tanager & $V * P R$ & AMNH, CLO, LSUMZ, MUSM & \\
\hline Haplospiza rustica (von Tschudi, 1844) & Slaty Finch & $\mathrm{v}$ & LSUMZ & \\
\hline Eucometis penicillata (von Spix, 1825) & Gray-headed Tanager & $\mathrm{V} * \mathrm{PR}$ & CLO, FMNH, LSUMZ, MCZ, MUSM & \\
\hline Dubusia taeniata (Boissonneau, 1840) & Buff-breasted Mountain-Tanager & $\mathrm{v}$ & LSUMZ & \\
\hline Diglossa glauca Sclater \& Salvin, 1876 & Deep-blue Flower-piercer & $P R$ & & \\
\hline Diglossa caerulescens (Sclater, PL, 1856) & Bluish Flower-piercer & VPR & LSUMZ, MUSM & \\
\hline Dacnis lineata (Gmelin, JF, 1789) & Black-faced Dacnis & $V * P R$ & FMNH, LSUMZ, MUSM, ROM, USNM & \\
\hline Dacnis flaviventer d'Orbigny \& Lafresnaye, 1837 & Yellow-bellied Dacnis & $V * P R$ & CLO, CM, FMNH, LSUMZ, MCZ, MUSM, UMMZ & \\
\hline Dacnis cayana (Linnaeus, 1766) & Blue Dacnis & $V * P R$ & CLO, FMNH, LSUMZ, MIZ, MUSM, USNM & \\
\hline Dacnis albiventris (Sclater, PL, 1852) & White-bellied Dacnis & VPR & FMNH, LSUMZ, MUSM & \\
\hline Cyanerpes nitidus (Hartlaub, 1847) & Short-billed Honeycreeper & VPR & FMNH, LSUMZ, MUSM & \\
\hline Cyanerpes cyaneus (Linnaeus, 1766) & Red-legged Honeycreeper & VPR & LSUMZ, MUSM & \\
\hline Cyanerpes caeruleus Linnaeus, 1758 & Purple Honeycreeper & $V * P R$ & LSUMZ, MCZ, MUSM, ROM, USNM & \\
\hline Coryphospingus cucullatus (Statius Muller, 1776) & Red-crested Finch & $\mathrm{PE}$ & & \\
\hline Conothraupis speculigera (Gould, 1855) & Black-and-white Tanager & $\mathrm{VP}$ & LSUMZ & IUCN-NT, MIN-NT \\
\hline Conirostrum speciosum (Temminck, 1824) & Chestnut-vented Conebill & $P$ & & \\
\hline Conirostrum margaritae (Holt, 1931) & Pearly-breasted Conebill & VPR & CLO, LSUMZ, MUSM, UF-FLMNH & IUCN-VU \\
\hline Conirostrum bicolor (Vieillot, 1809) & Bicolored Conebill & VPR & LSUMZ, UMMZ & IUCN-NT \\
\hline Conirostrum albifrons Lafresnaye, 1842 & Capped Conebill & R & & \\
\hline Coereba flaveola (Linnaeus, 1758) & Bananaquit & $V * P R$ & LSUMZ, MUSM, NHMO, UMMZ & \\
\hline Cissopis leverianus (Gmelin, JF, 1788) & Magpie Tanager & VPR & CLO, FMNH, KU, LSUMZ, MUSM, YPM & \\
\hline Chlorornis riefferii (Boissonneau, 1840) & Grass-green Tanager & $\mathrm{v}$ & LSUMZ, MCZ & \\
\hline Chlorophanes spiza (Linnaeus, 1758) & Green Honeycreeper & $V * P R$ & CLO, FMNH, LSUMZ, MCZ, MUSM, ROM, USNM & \\
\hline Chlorochrysa calliparaea (von Tschudi, 1844) & Orange-eared Tanager & $\mathrm{VP}$ & LSUMZ & \\
\hline Chalcothraupis ruficervix (Prévost \& Des Murs, 1846) & Golden-naped Tanager & VPR & LSUMZ & \\
\hline Catamenia homochroa Sclater, PL, 1859 & Páramo Seedeater & $\mathrm{v}$ & LSUMZ & \\
\hline Calochaetes coccineus (Sclater, PL, 1858) & Vermilion Tanager & PR & & \\
\hline Buthraupis montana (d'Orbigny \& Lafresnaye, 1837) & Hooded Mountain-Tanager & $\mathrm{v}$ & LSUMZ & \\
\hline Anisognathus somptuosus (Lesson, 1831) & Blue-winged Mountain-Tanager & VP & LSUMZ, MUSM, UF-FLMNH & \\
\hline Anisognathus lacrymosus (Du Bus, 1846) & Lacrimose Mountain-Tanager & $\mathrm{V}$ & LSUMZ & \\
\hline
\end{tabular}


Anexo 1. Colecciones científicas que tienen vouchers de aves de Loreto, Perú.

\begin{tabular}{|c|c|c|}
\hline Colección Científica & Acrónimo & País \\
\hline Cornell Lab of Ornithology, The Macaulay Library & CLO & EEUU \\
\hline American Museum of Natural History, New York. & AMNH & EEUU \\
\hline Biodiversity Research and Teaching Collections (BRTC), Texas A\&M University. & TCWC & EEUU \\
\hline California Academy of Sciences, San Francisco. & CAS & EEUU \\
\hline Cornell University Museum of Vertebrates, Ithaca. & CUMV & EEUU \\
\hline Denver Museum of Nature and Science, Denver. & DMNH & EEUU \\
\hline Field Museum of Natural History, Chicago. & FMNH & EEUU \\
\hline Louisiana State University Museum of Natural Science & LSUMZ & EEUU \\
\hline Museum of Comparative Zoology, Harvard University, Cambridge. & $\mathrm{MCZ}$ & EEUU \\
\hline Museum of Natural History and Science, Cincinnati Museum Center at Union Terminal & $\mathrm{CM}$ & EEUU \\
\hline Museum of Vertebrate Zoology, University of California, Berkeley. & MVZ & EEUU \\
\hline Natural History Museum of Los Angeles County, Los Angeles. & LACM & EEUU \\
\hline Occidental College, Moore Laboratory of Zoology, Los Angeles. & MLZ & EEUU \\
\hline Royal Ontario Museum, Department of Natural History, Toronto. & ROM & Canada \\
\hline Smithsonian Institution National Museum of Natural History (NMNH) & USNM & EEUU \\
\hline University of Florida, Florida Museum of Natural History & UF-FLMNH & EEUU \\
\hline University of Kansas Biodiversity Institute, Lawrence. & $\mathrm{KU}$ & EEUU \\
\hline University of Michigan Museum of Zoology, Ann Arbor. & UMMZ & EEUU \\
\hline University of Washington, Burke Museum of Natural History and Culture, Seattle. & UWBM & EEUU \\
\hline Western Foundation of Vertebrate Zoology, California. & WFVZ & EEUU \\
\hline Yale University, Peabody Museum of Natural History, New Haven. & YPM & EEUU \\
\hline Centro de Biodiversidad Naturalis de los Países Bajos & $\mathrm{RMNH}$ & Países Bajos \\
\hline Muséum national d'Histoire naturelle, Paris. & MNHN & Francia \\
\hline Zoologisk Museum, Universitetet i Oslo, Oslo. & NHMO & Noruega \\
\hline Museum and Institute of Zoology, Polish Academy of Sciences, Varsovia. & $\mathrm{MIZ}$ & Polonia \\
\hline Natural History Museum & $\mathrm{BMNH}$ & Reino Unido \\
\hline Branicki Zoological Museum, Varsovia. & MZBW & Polonia \\
\hline Universidad Nacional Mayor de San Marcos, Museo de Historia Natural & MUSM & Perú \\
\hline $\begin{array}{l}\text { Coleção Audiovisual do Museu de Zoologia "Adão José Cardoso" da UNICAMP- Fonoteca Neotropical Jacques } \\
\text { Vielliard (FNJV) }\end{array}$ & Unicamp-FNJV & Brasil \\
\hline Museu Paraense "Emilio Goeldi", Belém. & MPEG & Brasil \\
\hline
\end{tabular}

